\title{
ORIENTATIONS AND TRANSFERS IN COHOMOLOGY OF ALGEBRAIC VARIETIES
}

\author{
A. L. SMIRNOV
}

\begin{abstract}
Algebro-geometric cohomology theories are described axiomatically, with a systematic treatment of their orientations. For every oriented theory, transfer mappings are constructed for mappings of smooth varieties that are proper on supports. In some basic cases, transfers are calculated. The presentation is illustrated by motivic cohomology, $K$-theory, algebraic cobordism, and other examples.
\end{abstract}

The present paper concerns cohomology theories for algebraic varieties. Among these we can name étale cohomology and algebraic $K$-theory, known for a long time, as well as more recent theories such as motivic cohomology and algebraic cobordism.

In topology, a cohomology theory is described either by a representing spectrum in the stable homotopy category or axiomatically. By Brown's representability theorem, these approaches are equivalent. An algebraic counterpart of the stable homotopy category was found by Voevodsky and Morel in [1] and [2]. In the present paper, we present an algebraic analog of the Steenrod-Eilenberg axioms.

Our main goal in this paper is to study oriented theories. In topology, an orientation of a theory is a set of data that makes it possible to choose compatible fundamental classes (orientations) of the fibers of spherical bundles related to complex vector bundles. The same approach is used in the present paper, though a more conceptual definition of orientation would have been obtained by introducing a module structure over cobordism theory.

An essential advantage of oriented theories in topology is the computability of cohomology and related operations, e.g., the characteristic classes and transfers. In this paper, we present an algebraic version of these operations and develop appropriate computational tools. To apply these tools, it is necessary to know how to orient specific theories. For this, we present a natural orientation of algebraic cobordism and describe all orientations of an arbitrary theory (from this description it follows, in particular, that a theory with Chern classes is orientable). Furthermore, we introduce the notion of a transfer (an analog of the integral for cohomology classes as functions on spaces) and prove the main result, namely, the existence of a transfer for an orientable theory.

Now, we describe the content of the present paper in more detail. In Subsection 1.2. we define a cohomology theory and give basic examples; in Subsection 1.3, we present general properties of cohomology theories and describe some constructions (of considerable importance here is the deformation to the normal cone, see Subsection 1.3.6); in Subsection 1.4 we introduce products; in Subsections 1.5 and 1.6 we discuss suspension and group structures.

2000 Mathematics Subject Classification. Primary 14F99.

Key words and phrases. Algebraic variety, motivic cohomology, algebraic cobordism, orientation, transfer, characteristic class, residue.

Partially supported by RFBR (grant no. 03-01-00633a). 
In Subsection 2.2, we introduce orientations and the Thom operations; in Subsection 2.2.10, we describe the orientation of algebraic cobordism. In Subsection 2.3, we introduce the Gysin operators for a nonoriented theory and prove some properties of these operators (in particular, the functoriality in Subsection 2.3.7 which is used in the construction of transfers, though it follows from their properties). In Subsection 2.5, a theory with parameters is equipped with Chern classes; among their properties we prove, in particular, an important property of nilpotency. In Subsection 2.6. we identify the set of orientations of a theory with the set of parameters. In Subsection 2.7, we introduce the formal group of an oriented theory and also the formal group law and the invariant form determined by the choice of an orientation.

In Subsection 3.1, we introduce the notion of transfer and its compatibility with an orientation. In Subsection 3.2, we calculate transfers in some important cases; namely, in Subsection 3.2.1 we establish the relationship between the Gysin operators and Chern classes, in Subsection 3.2.4 we calculate the diagonal of a projective space, in Subsection 3.2.6 we prove the Quillen formula for projective bundles, in Subsections 3.2.7 and 3.2.8 we present the Mishchenko formula for the logarithm of a formal group law and the corresponding formula for the invariant form, in Subsection 3.2.9 we obtain a formula for transfers under blowups, and in Subsection 3.2.10, we compare the fundamental classes of fibers in a family. In Subsection 3.3, we equip an oriented theory with transfers.

In the Appendix, we collect auxiliary information: in Subsection 4.1, we list some properties of the target category for a cohomology theory and discuss the applicability of the theory to the case of a triangulated category; in Subsection 4.2. we outline the theory of the secondary deformation to the normal; in Subsection 4.3, we refine the notion of an infinite-dimensional variety; in Subsection 4.4 we present some properties of vector bundles (in particular, we construct the universal bundle and section); finally, in Subsection 4.5, we list the properties of residues used in the main body of the paper.

This paper is an extended and revised exposition of a part of the preprint $[3$ by I. Panin and the author. The new material contains, in particular, the notion of transfer with supports and its construction, the notion of an affine parameter and the residue invariance theorem, the theory of the secondary deformation to the normal cone, the calculation of transfers for blowups of the zeros of sections, the comparison of the fundamental classes of the fibers in families, and the example of a quaternionic topological theory.

A considerable part of the present paper was prepared during my stay at the Institute for Advanced Studies in Princeton in 2004-2005. I want to thank the Institute for support and hospitality.

\section{§1. Preliminary information}

We use the following terminology: a variety is an arbitrary (in particular, singular) quasiprojective variety over a fixed field $k, \mathrm{pt}=\operatorname{Spec} k$; all embeddings of varieties are locally closed; by a smooth variety we mean an equidimensional smooth variety; a vector bundle is a vector bundle of constant rank.

\subsection{Notation and conventions.}

- The category $\mathcal{S}$ of spaces under study consists of pairs $(X, U)$, where $X$ is a smooth variety and $U$ is open in $X$ (the space $(X, U)$ can be regarded as a homotopy quotient $X / U)$. A morphism $(X, U) \rightarrow(Y, V)$ is a mapping $f: X \rightarrow Y$ with $f(U) \subset V$. A variety $X$ is regarded as the pair $(X, \varnothing)$.

- For a space $P=(X, U) \in \mathcal{S}$, put $X_{P}=X$ and $U_{P}=U$. Let $j_{P}: U \rightarrow X$ be an open embedding, and $i_{P}:(X, \varnothing) \rightarrow(X, U)$ the identity mapping on $X$. 
- $\mathbf{P} E$ is the space of lines of a vector bundle $E ; E^{\vee}$ is the vector bundle dual to E.

- A support in $X$ is a closed subvariety $S \subset X$; a mapping from $X$ is called $S$-proper if its restriction to $S$ is proper.

- By s.e., ex, and nd, we denote the support extension operator (see Subsection 1.2.2), the excision operator (see Subsection 1.2.2), and the operator of deformation to the normal (see Subsections 1.3.6, 1.3.7).

- $R=A(\mathrm{pt})$ and $C=A\left(\mathbf{A}^{1}, \mathbf{A}^{1}-0\right)$ (see Subsection 1.5) .

1.2. Cohomology theories. The axioms of a cohomology theory for algebraic varieties are similar to the Steenrod-Eilenberg axioms for topological cohomology theories. The only axiom that has a nontrivial counterpart in algebraic geometry is the excision axiom. The flexibility of algebraic varieties necessary for a successful homotopy theory is provided by this axiom, and in particular by the fact that the étale excision is used rather than, e.g., the Zariski excision.

1.2.1. Target category. As the target category for a cohomology theory, we use an arbitrary category with exact triangles (see Subsection 4.1). For example, $\mathcal{A}$ can be a category of graded objects of an Abelian category with shift $M[1]^{i}=M^{i+1}$, where a triangle is exact if the sequences induced by it in each grading are exact. In particular, $\mathcal{A}$ can be the category of graded modules over a commutative ring $\Lambda$ or the category of bigraded $\Lambda$-modules with the shift $M[1]^{i, j}=M^{i+1, j}$.

1.2.2. Definition. A cohomology theory consists of a contravariant functor $A: \mathcal{S} \rightarrow \mathcal{A}$ and a morphism of functors $\partial_{P}: A\left(U_{P}\right) \rightarrow A(P)[1]$ (the boundary operator) for which the following properties are valid:

1) localization: $A(P) \stackrel{i_{P}^{*}}{\longrightarrow} A\left(X_{P}\right) \stackrel{j_{P}^{*}}{\longrightarrow} A\left(U_{P}\right) \stackrel{\partial_{P}}{\longrightarrow} A(P)[1]$ is an exact triangle (the triangle of a pair), where $f^{*}=A(f)$ for an arbitrary mapping $f$.

2) excision: the operator $A(X, U) \rightarrow A(\tilde{X}, \tilde{U})$ induced by a morphism $e:(\tilde{X}, \tilde{U}) \rightarrow$ $(X, U)$ is an isomorphism if $e: \tilde{X} \rightarrow X$ is étale at all points of $\tilde{Z}=\tilde{X}-\tilde{U}$ and induces an isomorphism between $\tilde{Z}$ and $Z=X-U$.

3) homotopy invariance: the operator $A(X) \rightarrow A\left(X \times \mathbf{A}^{1}\right)$ induced by the projection $X \times \mathbf{A}^{1} \rightarrow X$ is an isomorphism.

For a pair $(X, U)$, the operator $i_{P}^{*}: A(P) \rightarrow A\left(X_{P}\right)$ is denoted by s.e. and called the support extension operator. The object $A(X, U)$ is denoted by $A_{S}(X)$, where $S=X-U$, and is called the cohomology of $X$ with supports in $S$.

The examples we sketch below are used to illustrate the general theory. They become more specific as additional structures arise. In all examples, $\mathcal{A}$ is the category of graded Abelian groups.

1.2.3. Étale cohomology. Let $F$ be a locally constant torsion sheaf (a locally constant $l$-adic sheaf) on the étale $k$-site (see [4, II.1]). We assume that the torsion of $F$ (respectively, of $l)$ is prime to the characteristic of $k$. Let $A^{n}(X, U)=H_{S}^{n}\left(X_{\text {et }}, F\right)$ [4, 3.1], where $S=X-U$ and the boundary operator $\partial$ is defined in [4, 3.1.25]. The pair $(A, \partial)$ has the localization property [4,3.1.25], the excision property [4,3.1.27], and the homotopy invariance property [4, 6.4.20].

1.2.4. $K$-theory. Algebraic $K$-theory satisfies Definition 1.2.2. Namely, for each pair $P=(X, U)$, we consider the spectrum $K(P)=K^{\text {naive }}\left(X\right.$ on $\left.Z_{P}\right)$ [5, 3.1 and 1.5.2], where $Z=X-U$, and put $A^{n}(P)=\pi_{-n} K(P)$. The morphism $K(f)$ is defined by the functor $f^{*}$ [5, 3.14 and 3.16.7]. We note that the spectrum $K(P)$ is $(-1)$-connected, i.e., $\pi_{n} K(P)=0$ for $n<0$ [5, 1.5.3]. Therefore, $A^{n}(P)=0$ for $n>0$. 
For $n \leq 0$, the group $A^{n}(X)$ is isomorphic [5, 3.10] to the Quillen group $K_{-n}^{Q}(X)$, which shows, in particular, that $A$ is homotopy invariant in the case where $X$ is smooth. The excision property follows from [5, 3.19]. As to the boundary operator, the choice of it is not fixed in [5] explicitly, though the existence of a $\partial$ for which localization is valid follows from [5, 5.1] and the fact that $\pi_{0}\left(r_{0}\right): K_{0}(X) \rightarrow K_{0}(U)$ is epimorphic if $X$ is smooth [6, Exercise 5.15].

1.2.5. Representable ordinary theories. This example is based on 7 ; however, instead of the triangulated motivic category $D M^{-}(k)$, we employ the equivalent triangulated category $\mathcal{D M}^{-}$(it was used in [7, but no notation for it was introduced). Besides $\mathcal{D} \mathcal{M}^{-}$, we need the covariant functor $\mathbf{Z}_{\mathrm{tr}}: \mathcal{S} \rightarrow \mathcal{D} \mathcal{M}^{-}$and the morphism of functors $d: \mathbf{Z}_{\mathrm{tr}}(X, U) \rightarrow \mathbf{Z}_{\mathrm{tr}}(U)[1]$ in $\mathcal{D} \mathcal{M}^{-}$for which the following conditions are satisfied: (a) the triangle $\mathbf{Z}_{\mathrm{tr}}(U) \rightarrow \mathbf{Z}_{\mathrm{tr}}(X) \rightarrow \mathbf{Z}_{\mathrm{tr}}(X, U) \stackrel{d}{\rightarrow} \mathbf{Z}_{\mathrm{tr}}(U)[1]$ is distinguished in $\mathcal{D} \mathcal{M}^{-}$; (b) the morphism $\mathbf{Z}_{\mathrm{tr}}(\tilde{X}, \tilde{U}) \rightarrow \mathbf{Z}_{\mathrm{tr}}(X, U)$ induced by the morphism $e$ from the excision property in Definition 1.2 .2 is an isomorphism in $\mathcal{D M}^{-}$(excision); (c) the morphism $\mathbf{Z}_{\mathrm{tr}}\left(X \times \mathbf{A}^{1}\right) \rightarrow \mathbf{Z}_{\mathrm{tr}}(X)$ induced by the projection $X \times \mathbf{A}^{1} \rightarrow X$ is an isomorphism in $\mathcal{D} \mathcal{M}^{-}$(homotopy invariance).

With these data, we fix an arbitrary $F \in \mathcal{D M}^{-}$and put

$$
A^{n}(X, U)=\operatorname{Hom}_{\mathcal{D} \mathcal{M}^{-}}\left(\mathbf{Z}_{\mathrm{tr}}(X, U), F[n]\right) .
$$

As $\partial$, we take the operator $A^{n}(U) \rightarrow A^{n+1}(X, U)$ obtained by application of the functor $\operatorname{Hom}_{\mathcal{D M}^{-}}(*, F[n])$ to the morphism $d: \mathbf{Z}_{\mathrm{tr}}(X, U) \rightarrow \mathbf{Z}_{\mathrm{tr}}(U)[1]$. The properties in Definition 1.2 .2 follow directly from properties (a), (b), and (c). Now, we proceed to the data $\mathcal{D} \mathcal{M}^{-}, \mathbf{Z}_{\mathrm{tr}}$, and $\partial$ and their properties. The category $\mathcal{D} \mathcal{M}^{-}$and the functor $\mathbf{Z}_{\mathrm{tr}}$ are constructed along with the following sequence of functors and categories:

$$
\mathbf{Z}_{\mathrm{tr}}: \mathcal{S} \rightarrow \mathcal{N} S w T \rightarrow D^{-}(\mathcal{N} S w T) \rightarrow \mathcal{D} \mathcal{M}^{-} .
$$

Here, $\mathbf{Z}_{\text {tr }}$ may denote either a functor from $\mathcal{S}$ to $\mathcal{N} S w T$ or a functor from $\mathcal{S}$ to $D^{-}(\mathcal{N} S w T)$ or to $\mathcal{D} \mathcal{M}^{-}$obtained by composing $\mathbf{Z}_{\text {tr }}$ with arrows in (1). The category $\mathcal{N} S w T$ is a category of sheafs of Abelian groups with transfers on $\mathcal{S} m$ with respect to the Nisnevich topology [7, the 1st and 2nd paragraphs in $\S 1$ ], which is Abelian [7, the paragraph next to Lemma 1.2] ; $D^{-}(\mathcal{N} S w T)$ is the derived category for the category of (+1)-complexes over $\mathcal{N} S w T$ bounded from the right; $\mathcal{D M}^{-}$is the localization of $D^{-}(\mathcal{N} S w T)$ with respect to the triangulated subcategory of $\mathcal{A}$ weakly generated [7, 1.14] by the sheafs $\mathbf{Z}_{\mathrm{tr}}\left(X \times \mathbf{A}^{1}\right) / \mathbf{Z}_{\mathrm{tr}}(X \times 0)$.

The functor $\mathbf{Z}_{\mathrm{tr}}$ on the category $\mathcal{S}$ is defined by the formula $\mathbf{Z}_{\mathrm{tr}}(X, U)=\mathbf{Z}_{\mathrm{tr}}(X) /$ $\mathbf{Z}_{\mathrm{tr}}(U)$, where $\mathbf{Z}_{\mathrm{tr}}: \mathcal{S} m \rightarrow \mathcal{N} S w T$ is the functor defined in [7, the 3rd paragraph in $\left.\S 1\right]$.

The construction of $d$ and the proof that the triangle is distinguished are carried out at the level of $D^{-}(\mathcal{N} S w T)$. We consider the complex Cone $=\left[0 \rightarrow \mathbf{Z}_{\mathrm{tr}}(U) \rightarrow \mathbf{Z}_{\mathrm{tr}}(X) \rightarrow 0\right]$, where $\mathbf{Z}_{\mathrm{tr}}(X)$ is in degree 0 , and the morphism Cone $\rightarrow \mathbf{Z}_{\mathrm{tr}}(U)[1]$, which is the identity in degree $(-1)$ and is trivial in the other degrees. We define $d$ as the composition of $g^{-1}$ and the morphism Cone $\rightarrow \mathbf{Z}_{\mathrm{tr}}(U)[1]$, where the morphism $g:$ Cone $\rightarrow \mathbf{Z}_{\mathrm{tr}}(X) / \mathbf{Z}_{\mathrm{tr}}(U)$ is the natural projection in degree 0 and is trivial in the other degrees. The statements that $g$ is invertible and the triangle is distinguished in the derived category $D^{-}(\mathcal{N} S w T)$ are standard facts about derived categories.

As to the other properties of $A$, the excision property is checked in the proof of Lemma 4.11 in [7. (see also Subsection 1.3.1), and the property of homotopy invariance follows directly from the descriptions of $\mathcal{D} \mathcal{M}^{-}$and $\mathcal{A}$ given above.

The relationship between $\mathcal{D} \mathcal{M}^{-}$and the triangulated motivic category $D M^{-}(k)$ defined in [7, the paragraph before Theorem 1.5] as the complete subcategory of $D^{-}(\mathcal{N} S w T)$ 
consisting of complexes with homotopy invariant sheafs of cohomology, is described by the following diagram of categories and functors:

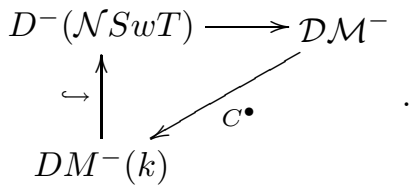

The equivalence of the categories $\mathcal{D M}^{-}$and $D M^{-}(k)$ (see [7, Theorem 1.12]) is given, in one direction, by the functor $C^{\bullet}$ (see [7, the paragraph before Theorem 1.12]) and, in the other direction, by the composition of the inclusion $D M^{-}(k) \hookrightarrow D^{-}(\mathcal{N} S w T)$ and the localization functor $D^{-}(\mathcal{N} S w T) \rightarrow \mathcal{D} \mathcal{M}^{-}$.

1.2.6. Representable extraordinary theories. This example is based on 2 . We need the triangulated category $\mathcal{S H}$ (the stable homotopy category from [2, Definition 5.7]), a functor $\mathcal{S} \rightarrow \mathcal{S H}$ that assigns an object denoted by $X_{+} / U_{+}$(also $X_{+}$for $U=\varnothing$ and $X / U$ in the general case) to each pair $(X, U)$, and a morphism of functors $d: X / U \rightarrow$ $U_{+}[1]$ with the following properties: (a) localization: the triangle $U_{+} \rightarrow X X_{+} \rightarrow X / U \stackrel{d}{\rightarrow}$ $U_{+}[1]$ is distinguished in $\mathcal{S H}$; (b) excision: the morphism $\tilde{X} / \tilde{U} \rightarrow X / U$ induced by the morphism $e$ from the excision property in Definition 1.2 .2 is an isomorphism in $\mathcal{S H}$; (c) homotopy invariance: the morphism $\left(X \times \mathbf{A}^{1}\right)_{+} \rightarrow X_{+}$induced by the projection $X \times \mathbf{A}^{1} \rightarrow X$ is an isomorphism in $\mathcal{S H}$.

Given these data, we fix an arbitrary $F \in \mathcal{S H}$ and put $A^{n}(X, U)=\operatorname{Hom}_{\mathcal{S H}}(X / U, F[n])$. As $\partial$, we take the operator $A^{n}(U) \rightarrow A^{n+1}(X, U)$ obtained by applying the functor $\operatorname{Hom}_{\mathcal{S H}}(*, F[n])$ to $d: X / U \rightarrow U_{+}[1]$. The properties listed in Definition 1.2 .2 follow directly from (a), (b), and (c). Now, we proceed to the necessary data and their properties. The functor $\mathcal{S} \rightarrow \mathcal{S H}$ is the composition

$$
\mathcal{S} \rightarrow \mathcal{S} p c_{\bullet} \rightarrow \mathcal{S H},
$$

where $\mathcal{S} p c_{\bullet}$ is the category $\mathcal{S} p c$ of punctured spaces and the category of spaces is the category of sheafs of sets with respect to the Nisnevich topology on $\mathcal{S} m$ [2, p. 421].

The functor $\mathcal{S} \rightarrow \mathcal{S} p c_{\bullet}$ in (2) is determined by the functor $\mathcal{S} m \rightarrow \mathcal{S} p c$ that, to every variety, assigns the sheaf represented by this variety. By [2, the 1st paragraph on p. 421], this sheaf turns out to be a sheaf in the Nisnevich topology. More precisely, the functor $\mathcal{S} \rightarrow \mathcal{S} p c_{\bullet}$ takes the pair $(X, U)$ to the quotient sheaf $X_{+} / U_{+}$(punctured by $U_{+} / U_{+}$), where $X_{+}$is represented by the variety $X_{+}=X \oplus$ pt.

In its turn, the functor $\mathcal{S} p c_{\bullet} \rightarrow \mathcal{S H}$ in (2) is the composition

$$
\mathcal{S p c}_{\bullet} \rightarrow \mathcal{H}_{\bullet} \rightarrow \mathcal{S W} \stackrel{\Sigma^{\infty}}{\longrightarrow} \mathcal{S H}
$$

where $\mathcal{H}_{\bullet}$ is the homotopy category of punctured spaces [2, p. 424], $\mathcal{S W}$ is the stable homotopy category of Spanier-Whitehead [2, Definition 4.2], the functor $\mathcal{S} p c_{\bullet} \rightarrow \mathcal{H}_{\bullet}$ is induced by the functor $\mathcal{S} p c \rightarrow \mathcal{H}$ implied in the definition of $\mathcal{H}$ [2, Definition 3.5], and the functor $\mathcal{H}_{\bullet} \rightarrow \mathcal{S W}$ is the natural functor implied in the definition of $\mathcal{S W}$ [2, Definition 4.2]. The functor $\Sigma^{\infty}: \mathcal{S W} \rightarrow \mathcal{S H}$ was mentioned in [2, Definition 5.7].

The excision property is already valid at the level of $\mathcal{S} p c_{\bullet}$. This follows from the fact that, in the case where $e$ is étale, the pair $(U, \widetilde{X})$ is a Nisnevich cover of $X$ (see also Subsection 1.3.1). The homotopy invariance is also valid at the level of $\mathcal{H}$ and follows from the definition of $\mathcal{H}$ in $[2,3.5]$ and property 1 in the list of weak equivalences following this definition.

The construction of $d$ and the proof of the localization property are done at the 
level of the triangulated category $\mathcal{S W}$. We consider the cofibration sequence $U_{+} \stackrel{i}{\rightarrow}$ $X_{+} \rightarrow$ Cone $(i) \rightarrow U[1]$ (see [2, the paragraph before Definition 4.8]) and define $d$ as the composition of $g^{-1}$, where $g: \operatorname{Cone}(i) \rightarrow X_{+} / U_{+}$is the $\mathcal{H}_{\bullet}$-isomorphism given below, and the morphism Cone $(i) \rightarrow U[1]$ from the cofibration sequence. The localization property follows from the fact that cofibration sequences are distinguished in $\mathcal{S W}$ 2, Definition 4.8].

Here, $g$ is defined as follows. The cone Cone $(i)$ [2, p. 427, (10)] is the colimit of the system $X_{+} \stackrel{i}{\leftarrow} U_{+} \stackrel{\operatorname{id} \wedge 1}{\longrightarrow} U_{+} \wedge \Delta^{1}$, where $\Delta^{1}=\mathbf{A}^{1}$ and the $\wedge$-product was defined in [2, p. 421, the last paragraph]. Therefore, to construct $g$, it suffices to define morphisms $X_{+} \rightarrow X_{+} / U_{+}$and $U_{+} \wedge \Delta^{1} \rightarrow X_{+} / U_{+}$, the composition of which with the morphisms from the system in the definition of the cone induces the same morphism $U_{+} \rightarrow X_{+} / U_{+}$. As such morphisms, we take the natural projection $X_{+} \rightarrow X_{+} / U_{+}$and the projection to the distinguished point $U_{+} \wedge \Delta^{1} \rightarrow X_{+} / U_{+}$. The fact that $g$ is $\mathcal{H}_{\bullet}$-invertible easily follows from properties (1) and (3) on the list of weak equivalences [2, pp. 423-424] and the fact that $X_{+} / U_{+}$is the colimit of the system $X_{+} \stackrel{i}{\leftarrow} U_{+} \rightarrow$ pt [2, p. 421, (7)].

1.2.7. Topological theories. For $\mathrm{k} \subset \mathbb{R}$, let $A^{n}(X, U)=H^{n}(X(\mathbb{R}), U(\mathbb{R}) ; \mathbb{Z} / 2)$. For $\mathrm{k} \subset \mathbb{C}$, let $A^{n}(X, U)=H^{i}(X(\mathbb{C}), U(\mathbb{C}) ; \mathbb{Z})$. For $\mathrm{k} \subset \mathbb{R}$, let $A^{n}(X, U)=H^{n}(X(\mathbb{H}), U(\mathbb{H}) ; \mathbb{Z})$, where $X(\mathbb{H})$ consists of all pairs $(P, \varphi)$ in which $P$ is a closed point of $X, \varphi: \mathrm{k}_{P} \otimes_{\mathrm{k}} \mathbb{R} \rightarrow \mathbb{H}$ is a homomorphism of $\mathbb{R}$-algebras, and $k_{P}$ is the residue field. To define a topology on $X(\mathbb{H})$, we consider the set $T$ of triples $(P, \psi, \alpha)$, where $P$ is a closed point of $X$ and $\psi: \mathrm{k}_{P} \otimes_{\mathrm{k}} \mathbb{R} \rightarrow \mathbb{C}$ and $\alpha: \mathbb{C} \rightarrow \mathbb{H}$ are homomorphisms of $\mathbb{R}$-algebras. Here, $T=\{(P, \psi)\} \times\{\alpha\},\{(P, \psi)\}=X(\mathbb{C})$, and $\{\alpha\}$ is the sphere $S^{2}$. Thus, we have a topology on $T=X(\mathbb{C}) \times S^{2}$. The surjection $T \rightarrow X(\mathbb{H}),(P, \psi, \alpha) \rightarrow(P, \alpha \psi)$, induces the quotient topology on $X(\mathbb{H})$.

1.3. General properties of cohomology theories. Here, we present some properties of an arbitrary cohomology theory $A$.

1.3.1. It suffices to check the excision property (see Subsection 1.2.2) only in the case where $e$ is étale at all points of $\tilde{X}$. This follows from the fact that étale morphisms are open.

1.3.2. The localization property implies that $A_{\varnothing}(X)=A(X, X)=0$.

1.3.3. The triangle $A(X, U) \stackrel{j^{*}}{\rightarrow} A(X, V) \stackrel{i^{*}}{\rightarrow} A(U, V) \stackrel{\partial}{\rightarrow} A(X, U)[1]$, where $X \supset$ $U \supset V(U$ and $V$ are open), $j$ is the embedding $(X, V) \subset(X, U), i$ is the embedding $(U, V) \subset(X, V)$, and $\partial$ is the composition of the support extension $A(U, V) \rightarrow A(U)$ and the differential $A(U) \rightarrow A(X, U)[1]$, is exact. To show this, first we prove that the triangle $A(U) \stackrel{\alpha}{\rightarrow} A(X, U)[1]+A(V) \stackrel{\beta}{\rightarrow} A(X, V)[1] \stackrel{\gamma}{\rightarrow} A(U)[1]$, where $\alpha=\partial_{(X, U)}+u^{*}$, $u$ is the embedding $V \subset U, \beta=-j^{*}[1]+\partial_{(X, V)}$, and $\gamma$ is the composition $A(X, V)[1] \rightarrow$ $A(X)[1] \rightarrow A(U)[1]$ of the support extension and restriction, is exact. To this end, from the cone (which is exact by 1.2.1, 3) of the morphism of triangles of the pair induced by $j$, we "subtract" the triangle $A(X) \rightarrow A(X) \rightarrow 0 \rightarrow A(X)[1]$ (see [8, 1.2.4] and 1.2.1, 2). Second, from the cone (which is exact by 1.2.1 3) of the morphism of exact triangles

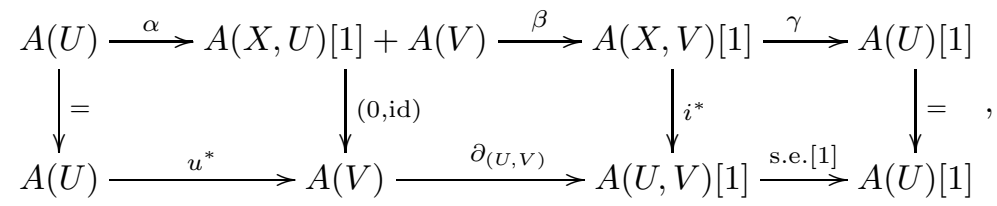

we "subtract" $A(U) \rightarrow 0 \rightarrow A(U)[1] \rightarrow A(U)[1]$ and $A(V) \rightarrow A(V) \rightarrow 0 \rightarrow A(V)[1]$. 
1.3.4. The following Mayer-Vietoris triangle is exact: $A(X, W) \stackrel{\rho}{\rightarrow} A(U, W) \oplus A(V, W) \stackrel{\alpha}{\rightarrow}$ $A(U \cap V, W) \stackrel{\delta}{\rightarrow} A(X, W)[1]$, where the pair $U, V$ is an open cover of $X, W$ is open in $U \cap V, \rho=j_{1}^{*}+j_{2}^{*}, \alpha=i_{2}^{*}-i_{1}^{*}\left(j_{1}: U \rightarrow X, j_{2}: V \rightarrow X, i_{1}: U \cap V \rightarrow U\right.$, and $i_{2}: U \cap V \rightarrow V$ are natural embeddings $), \delta=w^{*}[1] \circ\left(e^{*}[1]\right)^{-1} \circ \partial, \partial$ is the boundary operator of the triple $(U, U \cap V, W)$ (see Subsection 1.3.3), and $e:(U, U \cap V) \subset(X, V)$ and $w:(X, W) \subset(X, V)$ are natural inclusions ( $e^{*}$ is invertible by excision). To prove this, we consider the mapping of the triples $(U, U \cap V, W) \rightarrow(X, V, W)$ and the cone of the morphism of the corresponding exact triangles (this cone is exact by 1.2.1 3). From this cone, we "subtract" (see Subsection 1.3 .3 and [8, 1.2.4]) the triangle $0 \rightarrow A(X, V)[1] \rightarrow A(U, U \cap V)$, which is exact by excision.

Let the pair $U_{1}, U_{2}$ be an open cover of $X, S$ a support in $X, Y=S \cap U_{1}, Z=$ $S \cap U_{2}$, and $T=S \cap U_{1} \cap U_{2}$. For $W=X-S, U=U_{1} \cup W$, and $V=U_{2} \cup W$, applying excision to the inclusions $\left(U_{1}, U_{1} \cap W\right) \subset(U, W),\left(U_{2}, U_{2} \cap W\right) \subset(V, W)$, and $\left(U_{1} \cap U_{2}, U_{1} \cap U_{2} \cap W\right) \subset(U \cap V, W)$, we transform the Mayer-Vietoris triangle into the exact triangle

$$
A_{S}(X) \rightarrow A_{Y}\left(U_{1}\right) \oplus A_{Z}\left(U_{2}\right) \rightarrow A_{T}\left(U_{1} \cap U_{2}\right) \rightarrow A_{S}(X)[1] .
$$

1.3.5. The projection to the base and the section of an affine bundle induce isomorphisms in cohomology. Indeed, the affine bundle $p: T \rightarrow X$ is locally isomorphic to $X \times \mathbf{A}^{n}$, so that $p^{*}$ is an isomorphism by the homotopy invariance property (see Subsection 1.2.2). The property of $p^{*}$ being a global isomorphism reduces to a local property by the Mayer-Vietoris triangle. The section $s: X \rightarrow T$ induces an isomorphism in cohomology because $p s=\mathrm{id}$, and $p^{*}$ and $\mathrm{id}^{*}$ are isomorphisms.

1.3.6. The mappings $s$ and $g$ of the special and typical fibers of the deformation to the normal (see Subsection 4.2) induce isomorphisms in cohomology. Since, essentially, this was proved in [1, 3.2.4], we only outline the arguments. First, by direct comparison, the required property is verified for the pair $(X, Y)=\left(W \times \mathbf{A}^{n}, W \times 0\right)$ [1, 3.2.22]. Next, this property is proved for a pair $(X, Y)$ equipped with an étale morphism $q: X \rightarrow \mathbf{A}^{n}$ such that $Y=q^{-1}\left(\mathbf{A}^{m} \times 0\right)$ [1, 3.2.24]. In this proof, the "diagonal trick" is used to construct the diagram $X \stackrel{p_{1}}{\longleftarrow} \widetilde{X} \stackrel{p_{2}}{\longrightarrow} Y \times \mathbf{A}^{k}(k=n-m)$ for which $p_{1}$ and $p_{2}$ are étale and the mappings $p_{1}^{-1}(Y) \rightarrow Y$ and $p_{2}^{-1}(Y) \rightarrow Y \times 0$ are isomorphisms. Thus, $p_{1}$ and $p_{2}$ satisfy the excision property from Subsection 1.2.2, which makes it possible to reduce this case to the previous one. Finally, choosing a finite cover of $X$ by open sets $U$ such that $(U, U \cap Y)$ satisfy the conditions from the previous case, we can reduce the general case to the previous one.

Therefore, we have the isomorphism of the deformation to the normal

$$
A_{Y}\left(N_{Y} X\right) \stackrel{\text { nd }}{\longrightarrow} A_{Y}(X)
$$

determined by the composition $A_{Y}\left(N_{Y} X\right) \stackrel{\left(s^{*}\right)^{-1}}{\longrightarrow} A_{Y \times \mathbf{A}^{1}}\left(D_{Y} X\right) \stackrel{g^{*}}{\longrightarrow} A_{Y}(X)$.

1.3.7. For a support $S \subset Y$ and an $S$-proper (see Subsection 1.1) embedding of a smooth variety $Y$ into a smooth variety $X$, we have an isomorphism of the deformation to the normal with support

$$
A_{S}\left(N_{Y} X\right) \stackrel{\mathrm{nd}}{\longrightarrow} A_{S}(X) .
$$

It suffices to define (5) for $Y=\bar{Y}\left(\bar{Y}\right.$ is the closure of $Y$ ) because $N_{Y} X_{0}=N_{Y} X$ and, by excision, we have $A_{S}\left(X_{0}\right)=A_{S}(X)$, where $X_{0}=X-Z$ and $Z=\bar{Y}-Y$. In this case (in

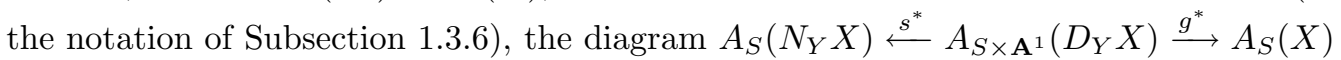
consists of isomorphisms, where $S \times \mathbf{A}^{1} \subset D_{Y} X$ as a part of $Y \times \mathbf{A}^{1}$, and nd $=g^{*}\left(s^{*}\right)^{-1}$. 
The invertibility of $s^{*}$ and $g^{*}$ is proved by comparing the diagrams with support and without support and taking into account the exactness of the triangle of the triple, the 5-lemma, and the excision isomorphisms $A_{Y-S}\left(N_{Y} X-S\right)=A_{Y-S}\left(N_{Y-S}(X-S)\right)$ and $A_{Y \times \mathbf{A}^{1}-S \times \mathbf{A}^{1}}\left(D_{Y} X-S \times \mathbf{A}^{1}\right)=A_{(Y-S) \times \mathbf{A}^{1}}\left(D_{Y-S}(X-S)\right)$.

1.3.8. Under the natural identification $N_{X} X=X$, the operator $A_{S}(X) \stackrel{\text { nd }}{\longrightarrow} A_{S}\left(N_{X} X\right)$ for a variety $X$ and a support $S \subset X$ is the identity. Moreover, under the natural identification $N_{Y} X=Y$, for an isomorphism $i: Y \rightarrow X$ and a support $T \subset Y$, the operator nd: $A_{T}\left(N_{Y} X\right) \rightarrow A_{S}(X)$, where $i(T)=S$, is inverse to $i^{*}$. This follows from the fact that the deformation $D_{X} X$ is trivial (see Subsection 4.2.5).

1.3.9. For a support $S \subset Y$ and an $S$-proper embedding $i: Y \subset X$ of smooth varieties, the following diagram is commutative:

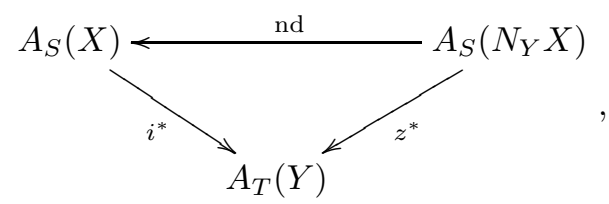

where $z: Y \rightarrow N_{Y} X$ is the zero section. This follows from the construction of nd (see Subsection 1.3.7) and the fact that $i^{*}$ and $z^{*}$ are fibers of the restriction $A_{S \times \mathbf{A}^{1}}\left(D_{Y} X\right) \rightarrow$ $A_{S \times \mathbf{A}^{1}}\left(D_{Y} Y\right)$.

1.3.10. If $Y \subset X$ is a closed embedding of smooth varieties and a mapping $f: \tilde{X} \rightarrow X$ is transversal to $Y$, then the diagram

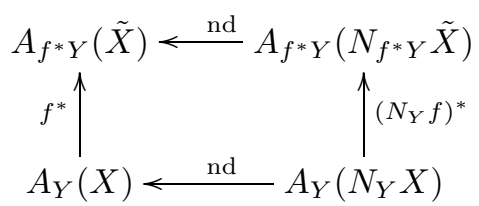

is commutative. This follows from the functoriality of the deformation to the normal, namely, from the fact that diagram (43) in Subsection 4.2.6 is commutative.

1.3.11. For a vector bundle $E / X$, the operator $\nu^{*}: A_{S}(E) \rightarrow A_{S}\left(N_{X} E\right)$ induced by the natural isomorphism $\nu: N_{E} \rightarrow E$ in (38) is inverse to the operator nd : $A_{S}\left(N_{X} E\right) \rightarrow$ $A_{S}(E)$. This follows from the fact that the deformation to the normal is trivial for the zero section of a vector bundle (see Subsection 4.2.10).

1.3.12. Under the conditions of Subsection 4.4.8 the operator $\left(\nu^{*}\right)^{-1}: A_{\mathbf{P} F}(N) \rightarrow$ $A_{\mathbf{P} F}(\mathbf{P} V-\mathbf{P} E)$ coincides with the isomorphism of the deformation to the normal for the embedding $\mathbf{P} F \subset \mathbf{P} V-\mathbf{P} E$ (this follows from 1.3.11). Thus, by Subsection 1.3.10, the isomorphism nd : $A_{\mathbf{P} F}(N) \rightarrow A_{\mathbf{P} F}(\mathbf{P} V)$ coincides with the composition $A_{\mathbf{P} F}(N) \stackrel{\left(\nu^{*}\right)^{-1}}{\longrightarrow}$ $A_{\mathbf{P} F}(\mathbf{P} V-\mathbf{P} E) \stackrel{\text { ex }}{\longrightarrow} A_{\mathbf{P} F}(\mathbf{P} V)$, and the triangle

$$
A_{\mathbf{P} F}(N) \stackrel{\text { s.e.ond }}{\longrightarrow} A(\mathbf{P} V) \rightarrow A(\mathbf{P} E) \rightarrow A_{\mathbf{P} F}(N)[1],
$$

obtained from the triangle of the pair $(\mathbf{P} V, \mathbf{P} V-\mathbf{P} F)$ by replacing $A_{\mathbf{P} F}(\mathbf{P} V)$ with $A_{\mathbf{P} F}(N)$ and $A(\mathbf{P} V-\mathbf{P} F)$ with $A(\mathbf{P} E)$, is exact (see Subsection 4.4.6). 
1.3.13. For closed embeddings $Z \subset Y \subset X$ of smooth varieties and a support $S \subset Z$, we have the commutative diagram

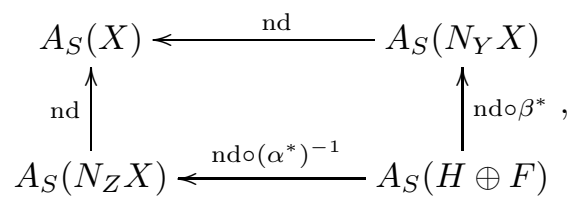

where $H=N_{Z} Y, F=N_{Z} X / N_{Z} Y$, and the natural isomorphisms $\alpha: N_{H} N_{Z} X \rightarrow H \oplus F$ and $\beta: H \oplus F \rightarrow N_{Z} N_{Y} X$ are defined in (39).

The commutativity of (9) is a consequence of the following facts: the diagram of the secondary deformation to the normal is commutative (see Subsection 4.2.11), the application of $A$ transforms all arrows in the diagram in Subsection 4.2.11 to isomorphisms, and the identifications $\alpha$ and $\beta$ are implemented appropriately into this diagram (see Subsection 4.2.12).

The support extension operator $A_{X}(E) \rightarrow A(E)$ is zero $(X$ is embedded into $E$ by the zero section) whenever there exists a section $s$ of $E / X$ without zeros, because the triangle of the pair $(E, E-X)$ is exact and the restriction $A(E) \rightarrow A(E-X)$ is monomorphic ( $s$ induces a splitting $E \rightarrow X \stackrel{s}{\rightarrow}(E-X)$ ).

1.3.14. The support extension operator $A_{\mathbf{P} E}(\mathbf{P}(E+1)) \rightarrow A(\mathbf{P}(E+1))$ is an embedding onto a direct summand. This follows from the exactness of the triangle of the pair $(\mathbf{P}(E+1), U)$ for $U=\mathbf{P}(E+1)-\mathbf{P} E$, the identification $A(U)=A(X)$ (see Subsection 4.4.8), and the existence of a section $X \rightarrow \mathbf{P}(E+1)$ that splits the restriction to $U$.

1.3.15. For embeddings $a, b: E \rightarrow V$ of vector bundles on $X$, there exists an embedding of vector bundles $i: V \rightarrow W$ such that $a^{*} \circ i^{*}=b^{*} \circ i^{*}: A(\mathbf{P} W) \rightarrow A(\mathbf{P} E)$. This follows from 4.4.5 and the homotopy invariance. Here, even for $X=\mathrm{pt}$, the mapping $a^{*}$ can depend on $a$. For example, the operator $a^{*}$ for the automorphism of $\mathbf{P}^{1}, x \mapsto-x$, is not the identity for the topological $\mathbb{R}$-theory with $\mathbb{Z}$-coefficients (see Subsection 1.2.7).

1.3.16. For vector bundles $E \subset V$ and $F=V / E$ on a smooth variety $X$, the projection $U=\mathbf{P} V-\mathbf{P} E \rightarrow \mathbf{P} F$ induces an isomorphism $A(\mathbf{P} F) \simeq A(U)$ because (see Subsection 1.3.5) there is a structure of an affine bundle on $U$ (see Subsection 4.4.6).

1.4. Multiplicative structures. To define a ring theory (see Subsection 1.4.3), the following formalism is convenient. On the category of spaces over a smooth $X$ (i.e., the category of mappings $P \rightarrow X$ ), there is a symmetric monoidal structure given by the product $P \times_{X} Q=\left(Y \times_{X} Z, Y \times_{X} W \cup V \times_{X} Z\right)$ for $P=(Y, V)$ and $Q=(Z, W)$ with permutation of the symmetric structure $(y, z) \mapsto(z, y)$. For $X=\mathrm{pt}$, we obtain the tensor structure $P \times Q$ on $\mathcal{S}$.

1.4.1. The target category of a ring theory. As the target category of a ring theory, we use the target category of a cohomology theory $\mathcal{A}$ (see Subsection 1.2.1) equipped with the structure of an additive symmetric monoidal category with unit $R$ and with left multiplication by the invertible object $R[1]$ in the role of the shift. In addition, we assume that the functor $M \mapsto \operatorname{Hom}(R, M)$ takes exact triangles to exact sequences. In the sequel, we assume that $\mathcal{A}$ is of that type.

For example, $\mathcal{A}$ can be the category of graded modules over a commutative graded ring $R$, i.e., over a commutative monoid in the symmetric monoidal category of graded Abelian groups with $\otimes=\otimes_{R}$ and the permutation $x \otimes y \mapsto(-1)^{\operatorname{deg} x \operatorname{deg} y} y \otimes x$ on the homogeneous elements. In the next example, $\mathcal{A}$ is the category of bigraded modules over a bigraded ring $R$, i.e., over a commutative monoid in the symmetric monoidal category 
of bigraded modules over a commutative ring $\Lambda$ with $\otimes=\otimes_{R}$ and the permutation on the homogeneous elements given by $x \otimes y \mapsto(-1)^{\operatorname{deg}_{1} x \operatorname{deg}_{1} y} \varepsilon^{\operatorname{deg}_{2} x \operatorname{deg}_{2} y} y \otimes x$, where $\varepsilon$ is the element of $\Lambda^{\times}$of order 2 .

1.4.2. Monoidal functors. A functor is weakly monoidal if it has all the properties of a monoidal functor [9, 4.1.2] except the invertibility of $F(X) \otimes F(Y) \rightarrow F(X \otimes Y)$. In other words, a weakly monoidal functor is a triple $(F, m, \alpha)$, where $F: \mathcal{C} \rightarrow \mathcal{D}$ is a functor of monoidal categories, $m: F(X) \otimes F(Y) \rightarrow F(X \otimes Y)$ is a morphism of functors, and $\alpha: F\left(1_{\mathcal{C}}\right) \rightarrow 1_{\mathcal{D}}$ is an isomorphism. It is required that two morphisms from $(F X \otimes F Y) \otimes F Z$ to $F(X \otimes(Y \otimes Z))$ coincide, two morphisms from $F\left(1_{\mathcal{C}}\right) \otimes F X$ to $F\left(1_{\mathcal{C}} \otimes X\right)$ coincide, and two morphisms from $F X \otimes F\left(1_{\mathcal{C}}\right)$ to $F\left(X \otimes 1_{\mathcal{C}}\right)$ coincide. If $\mathcal{C}$ and $\mathcal{D}$ are symmetric, then a weakly monoidal functor is said to be symmetric provided that $F\left(t_{\mathcal{C}}\right) m=m t_{\mathcal{D}}$, where $t_{\mathcal{C}}$ and $t_{\mathcal{D}}$ are permutations of the symmetric structures. Neglecting subtleties, we assume that $F\left(1_{\mathcal{C}}\right)=1_{\mathcal{D}}$, which allows us not to mention $\alpha$.

1.4.3. Definition. A cohomology theory $A$ is said to be a ring theory if $A$ has the structure of a weakly monoidal functor $m: A(P) \otimes A(Q) \rightarrow A(P \times Q)$ such that, for every pair $P=(X, U)$ and a smooth variety $Y$, the Leibnitz rule $\partial_{P} \otimes 1_{Y}=\partial_{P \times Y}$ and $1_{Y} \otimes \partial_{P}^{r}=\partial_{Y \times P}^{r}$ is fulfilled, where the right differential $\partial_{P}^{r}$ is defined by the composition $A(U) \stackrel{\partial_{P}}{\rightarrow} R[1] \otimes A(P) \stackrel{t}{\rightarrow} A(P) \otimes R[1]$ and $t$ is a permutation of the symmetric structure on $\mathcal{A}$. The ring theory is commutative if $A$ is a symmetric functor.

In what follows, by a ring theory we mean a commutative theory.

1.4.4. Intrinsic multiplication. In a ring theory, for all pairs $P, Q$ over a smooth variety $X$, the multiplication $m$ induces the multiplication $m_{X}=\Delta^{*} m: A(P) \otimes A(Q) \rightarrow$ $A\left(P \times_{X} Q\right)$, where $\Delta: P \otimes_{X} Q \rightarrow P \times Q$ is the diagonal. In particular, for a space $(Y, V)$, the mapping $f: Y \rightarrow X$ induces the structure of a two-sided $A(X)$-module on $A(Y, V)$.

Given $m_{X}$ restricted to the pairs $P, Q$ with $X_{P}=X_{Q}=X$ (the intrinsic multiplication), we can restore the multiplication $m=m_{Y \times Z}\left(y^{*} \times z^{*}\right)$, where $y$ and $z$ are projections from $Y \times Z$ onto $Y$ and $Z$, respectively.

1.4.5. For supports $S, T \subset Y$ and an embedding $i: Y \subset X$ of smooth varieties that is $S$-proper and $T$-proper, we have the commutative diagram

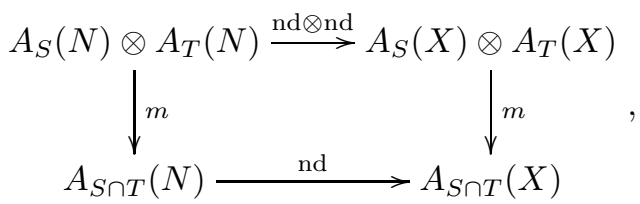

where $N=N_{Y} X$ and $m$ is the multiplication. This follows from the construction of nd (see Subsections 1.3.7 and 4.2.7).

1.4.6. Suppose $E_{1}, E_{2} / X$ are vector bundles and $E=E_{1}+E_{2}$. Next, for $D \in \mathcal{A}$, suppose that the functor $\operatorname{Hom}(D, *)$ takes exact triangles to exact sequences, and let $u_{1}, u_{2}: D \rightarrow A(\mathbf{P} E)$ be such that the restrictions of $u_{i}$ to $\mathbf{P} E_{i}$ are trivial. Then $u_{1} u_{2}=0$. This follows from the fact that $u_{1}$ and $u_{2}$ are support extensions for some $x_{1}: D \rightarrow A_{\mathbf{P} E_{2}}(\mathbf{P} E)$ and $x_{2}: D \rightarrow A_{\mathbf{P} E_{1}}(\mathbf{P} E)$ (this is a consequence of 1.3 .16 and the exactness of the triangle of a pair). Thus, $u_{1} u_{2}$ ie equal to s.e. $\left(x_{1} x_{2}\right)$, and is zero, because it comes from the support of $\mathbf{P} E_{1} \cap \mathbf{P} E_{2}=\varnothing$. 
1.4.7. A theory $A$ with a ring theory data satisfying all the properties in Definition 1.4.3 except for the identification of $A(\mathrm{pt})$ with $\Lambda$ (the unit of $\mathcal{A}$ ) extends to a ring theory with values in the category of two-sided $R$-modules $\mathcal{A}(R=A(\mathrm{pt}))$ provided $\mathcal{A}$ has finite colimits. In this case, the shift of an $R$-module $M$ is defined as $M[1]$ with multiplications $R \otimes M[1] \simeq R \otimes \Lambda[1] \otimes M \simeq \Lambda[1] \otimes R \otimes M \rightarrow \Lambda[1] \otimes M \simeq M[1]$ (a permutation is used) and $M[1] \otimes R \simeq \Lambda[1] \otimes M \otimes R \rightarrow \Lambda[1] \otimes M \simeq M[1]$; by definition, a triangle is exact if and only if it is exact in $\mathcal{A}$, and $M \otimes_{R} N$ is defined by the colimit $M \otimes N \leftarrow M \otimes R \otimes N \rightarrow M \otimes N$, where the arrows are given by multiplications. The relation $M[1] \simeq R[1] \otimes_{R} M$ follows from the fact that, by invertibility, the shift commutes with colimits.

In the examples given below, by default, the extension mentioned above is assumed to be performed.

1.4.8. Étale cohomology (see Subsection 1.2.3). For a sheaf of commutative rings $F$, the theory $A^{i}(X, U)=H_{S}^{i}\left(X_{\text {et }}, F\right)$ is ring with respect to the intrinsic multiplication defined by the pairing of sheafs $F \otimes F \rightarrow F$ [4, 5.1.17]. The compatibility of $\partial$ with the multiplication was proved in [4, 5.1.16]. This theory is commutative, i.e., $a b=(-1)^{m n} b a$; see [4, 5.1.18].

1.4.9. K-theory (see Subsection 1.2.4). The intrinsic multiplication was defined in $[5$, 3.15.4]. By [5, 3.15], we know that $K(X)$ is a "homotopy" ring spectrum with multiplication that is associative up to "compatible homotopies". This implies associativity. The fact that the multiplication is commutative and compatible with $\partial$ was proved in 10.

1.4.10. Representable ordinary theories (see Subsection 1.2.5). On $\mathcal{D} \mathcal{M}^{-}$, we have a tensor structure $A \otimes^{L} B$ with unit $\mathbf{Z}_{\mathrm{tr}}$ (pt) (see [7, 2.8]). The structure of a commutative monoid on $F \in \mathcal{S H}$ equips the theory $H_{\mathcal{M}}^{n}(P ; F)$ with an intrinsic multiplication. The Leibnitz rule reduces to the fact that the relations $d_{P} \times Y=d_{P \times Y}$ and $Y \times d_{P}=d_{Y \times P}$ are valid for a pair $P$ and a smooth variety $Y$. We skip the proof of these relations.

1.4.11. Representable extraordinary theories (see Subsection 1.2.6). By 2, Theorem 5.6], we have the tensor structure $X \wedge Y$ on $\mathcal{S H}$. The structure of a commutative monoid on $F \in \mathcal{S H}$ equips the theory $\operatorname{Hom}_{\mathcal{S H}}(X, U ; F[n])$ with an intrinsic multiplication. The Leibnitz rule reduces to the fact that the relations $d_{P} \times Y=d_{P \times Y}$ and $Y \times d_{P}=d_{Y \times P}$ are valid for a pair $P$ and a smooth variety $Y$. We skip the proof of these relations.

In what follows, $A$ is a commutative ring theory.

1.5. Algebraic suspension. As algebraic suspension we take the product with the sphere

$$
\Sigma_{a}=\left(\mathbf{A}^{1}, \mathbf{A}^{1}-0\right) .
$$

The algebraic suspension operator is the multiplication $\sigma: A\left(\Sigma_{a}\right) \otimes A(P) \rightarrow A\left(\Sigma_{a} \times P\right)$.

1.5.1. Other spheres. The sphere $\mathbf{G}_{m}$ is less convenient (the pair $\left(\mathbf{G}_{m}, 1\right)$ is not open) and does not change the situation essentially, because canonically $A\left(\mathbf{G}_{m} \times X\right) \simeq A(X)+$ $A\left(\Sigma_{a} \times X\right)[1]$. Here, the projection to $A(X)$ is given by the mapping $\mathbf{A}^{1} \rightarrow \mathbf{G}_{m}, x \mapsto 1$, which splits the triangle of the pair $\left(\mathbf{A}^{1}, \mathbf{G}_{m}\right) \times X$.

The sphere $\mathbf{P}^{1}$ is inconvenient for the same reasons. The sphere with the distinguished homotopy point $\left(\mathbf{P}^{1}, \mathbf{A}^{1}\right)$ is essentially the same as $\Sigma_{a}$, because $A\left(\mathbf{P}^{1} \times X\right) \simeq A(X)+$ $A\left(\Sigma_{a} \times X\right)$ and $A\left(\Sigma_{a} \times X\right) \simeq A\left(\left(\mathbf{P}^{1}, \mathbf{A}^{1}\right) \times X\right)$ by excision. Here, the projection to $A(X)$ is given by restriction to pt $\times X \subset \mathbf{P}^{1} \times X$, which splits the triangle of the pair $\left(\mathbf{P}^{1}, \mathbf{A}^{1}\right) \times X$. 
1.5.2. Definition. A space $X$ is said to be $A$-flat (or flat if the theory $A$ is clear from the context) if the multiplication $A(X) \otimes A(Y) \rightarrow A(X \times Y)$ is an isomorphism for all spaces $Y$ and the functor $M \mapsto A(X) \otimes M$ takes exact triangles to exact triangles. This terminology applies also to infinite-dimensional varieties $X$ (see Subsection 4.3).

The flatness of $\Sigma_{a}$ is equivalent to that of $\mathbf{P}^{1}$ or $\mathbf{G}_{m}$ (see Subsection 1.5.1). If $\Sigma_{a}$ is flat, then the decomposition in Subsection 1.5.1 turns into

$$
A\left(\mathbf{P}^{1} \times X\right) \simeq A(X)+C \otimes A(X) .
$$

1.5.3. Definition. A theory $A$ is said to be spherically stable if the sphere $\Sigma_{a}$ is flat and $C=A\left(\Sigma_{a}\right)$ is invertible.

For a spherically stable theory $A$, the operator $\operatorname{Hom}(M, N) \rightarrow \operatorname{Hom}(C \otimes M, C \otimes N)$, $\varphi \mapsto \mathrm{id} \otimes \varphi$, is an isomorphism for all $M, N \in \mathcal{A}$ and the functor $M \mapsto \operatorname{Hom}\left(C^{\otimes n}, M\right)$ takes exact triangles to exact sequences (see Subsection 1.4.1).

1.5.4. Stabilization. Starting with a theory with flat $\Sigma_{a}$, we construct a spherically stable theory $\mathcal{S} \rightarrow \mathcal{A} \rightarrow \mathcal{A}\left[C^{-1}\right]$, where $\mathcal{A}\left[C^{-1}\right]$ is the category described in [2, p. 426]. The condition under which the $\otimes$-structure extends to $\mathcal{A}\left[C^{-1}\right]$ (arising from the fact that the automorphism groups of invertible objects are Abelian), namely, the triviality of the natural action of the alternating group $A_{n}$ on $A\left(\Sigma_{a}^{\otimes n}\right)=C^{\otimes n}$, is fulfilled because the automorphism $\Sigma_{a} \otimes \Sigma_{a},(x, y) \mapsto(y,-x)$ is homotopic to the identity mapping, $(x, y, t) \mapsto((1-t) x+t y,-t x+(1+t) y)$. By definition, a triangle in $\mathcal{A}\left[C^{-1}\right]$ is exact if after the tensor multiplication by $C^{n}$ for some $n$ it becomes isomorphic to the image of an exact triangle from $\mathcal{A}$.

1.5.5. Twists. For a spherically stable theory, we have the twists

$$
M[p](q)=R[1]^{\otimes p} \otimes R(1)^{\otimes q} \otimes M, \quad M \in \mathcal{A} \quad(\text { or } M \in \operatorname{Pro} \mathcal{A}),
$$

where $R(-1)$ is determined by the equation $C=R[-2](-1)$, i.e., $R(-1)=R[2] \otimes C$.

For a monoid $S \in \mathcal{A}, N \in \mathcal{A}$, and a two-sided $S$-module $M$, the action of $S$ on $N \otimes M$ is defined as $S \otimes N \otimes M \rightarrow N \otimes S \otimes N \rightarrow N \otimes M$ and $N \otimes M \otimes S \rightarrow N \otimes M$. Thus, we have an action of $S$ on twists of the $S$-module.

In what follows, we use the following terminology. The operators $M \rightarrow N[p](q)$ are called homogeneous operators of degree $[p](q)$ from $M$ to $N$. With every smooth variety $X$ (which may be infinite-dimensional), we can associate the graded topological ring

$$
A^{\bullet}(X) \text {, where } A^{n}(X)=\operatorname{Hom}\left(C^{\otimes n}, A(X)\right)=\operatorname{Hom}(R, A(X)[2 n](n)) .
$$

The topology was defined in Subsection 4.3 .2 and is discrete for finite-dimensional varieties. Here, $f \in A^{n}(X)$ acts on $A(P)$ for a space $P$ over $X$ by an operator $[f]$ of degree $[2 n](n)$, namely, by the composition $C^{\otimes n} \otimes A(P) \stackrel{f \otimes 1}{\longrightarrow} A(X) \otimes A(P) \stackrel{\text { mult. }}{\longrightarrow} A(P)$, and $[f g]=[f] \circ[g]$. In particular, $A^{\bullet}(X)$ is an $R^{\bullet}$-algebra, where $R^{\bullet}=A^{\bullet}(\mathrm{pt})$.

A family of homogeneous operators $x_{i}: R \rightarrow M$ is called a basis of $M \in \operatorname{Pro} \mathcal{A}$ if, first, for each $m \in \mathbb{Z}$, the system of morphisms $X_{m, n}=\bigoplus C^{\operatorname{deg} x_{i}} \rightarrow M$ with $m \leq \operatorname{deg} x_{i} \leq n$ and the operator $\sum x_{i}$ extends to a morphism $X_{m} \rightarrow M$, where $X_{m}=\lim _{\longleftarrow} X_{m, n}, n \rightarrow \infty$, and second, the induced morphism $\lim _{m} X_{m} \rightarrow M, m \rightarrow-\infty$ is an isomorphism. For "free" $A(X)$-modules (i.e., for the modules that have bases), there is a tensor product (see Subsection 1.4.7) over $A(X)$.

Below, we present several examples.

1.5.6. Étale cohomology. We come back to the example in Subsection 1.4 .8 but now $F$ is graded, $F^{j}=\mu_{l}^{\otimes j}$, and $\mathcal{A}$ is the category of bigraded modules over a bigraded ring $R$ (see Subsection 1.4.1), where $R^{i, j}=H_{\mathrm{et}}^{i}\left(\mathrm{pt}, F^{j}\right)$. This theory is spherically stable (see the calculation of the cohomology of $\mathbf{P}^{1}$ [4, VI, 5.6] and Subsection [1.5.1). 
1.5.7. $K$-theory (see Subsection 1.4.9). Here, $\mathcal{A}$ is the category of graded modules over a graded $R$ with $R^{i}=K_{-i}(\mathrm{pt})$. This theory is spherically stable because $A\left(\Sigma_{a}\right)=A(\mathrm{pt})$ (for the calculation of the $K$-theory for $\mathbf{P}^{1}$, see [11, 8.3.1]). There is an isomorphism of twists $M[p](q) \rightarrow M[p+2 d](q+d)$ (see [2, 6.2]) given by the multiplication by $\beta^{d}$, where $\beta: R \rightarrow R[2](1)$ is a special case of the morphism indicated. Here, $\beta^{-1}$ induces an isomorphism $K_{0}(\mathrm{pt}) \rightarrow K_{0}\left(\mathbf{P}^{1}, \mathbf{A}^{1}\right)$ and is determined by it. For definiteness, we assume that this isomorphism takes [1] to [1] $-[\mathcal{O}(-1)]$ (in [2] $\beta$ was not fixed). Thus, $\beta^{-1}=[1]-[\mathcal{O}(-1)] \in A^{2,1}(\mathrm{pt})$, and $\beta \in A^{-2,-1}(\mathrm{pt})$ goes to $1 \in K_{0}(\mathrm{pt})$ under the identification $\beta^{-1}: A^{-2,-1}(\mathrm{pt}) \rightarrow A^{0,0}(\mathrm{pt})=K_{0}(\mathrm{pt})$.

1.5.8. Representable ordinary theories (see Subsection 1.4.10). Here, $\mathcal{A}$ is the category of bigraded modules over a bigraded ring $R$ (see Subsection 1.4.1), where

$$
R^{i, j}=H_{\mathcal{M}}^{i}\left(\mathrm{pt}, F^{j}\right), \quad F^{j}=F \otimes^{L} \mathbf{Z}(1)^{\otimes j}, \quad \text { and } \quad \mathbf{Z}(1)=\left(\mathbf{Z}_{\mathrm{tr}}\left(\mathbf{G}_{m}\right) / \mathbf{Z}\right)[-1]
$$

(see [7, 3.1] and Subsection [1.2.5). The theory $H_{\mathcal{M}}^{i}\left(X, F^{j}\right)$ is spherically stable at least in characteristic zero [7, 4.12.1]. We shall see that $\varepsilon=1$ (1.4.1) (a consequence of orientability, 2.6.4, and 2.2.4).

1.5.9. Representable extraordinary theories (see Subsection 1.4.11). Here, $\mathcal{A}$ is the category of bigraded modules over a bigraded ring $R$ (see Subsection 1.4.1), $A^{i, j}(X)=$ $\mathcal{S H}\left(X, \Sigma_{a}^{\otimes j}[-i] \wedge F\right), \Lambda=A^{0,0}(\mathrm{pt}), \varepsilon=u i_{0} \in \Lambda$, where $u:$ pt $\rightarrow F$ (the unit of the monoid), $i: \Sigma_{a} \rightarrow \Sigma_{a}, t \mapsto-t$, and $i_{0}=i \otimes \Sigma_{a}^{-1}$. This theory is spherically stable because suspensions are invertible in $\mathcal{S H}$.

1.5.10. Topological theories (see Subsection 1.2.7). Here, $\mathcal{A}$ is the category of graded modules over a graded ring $R$ with $R^{i}=A^{i}(\mathrm{pt})$. The theories in Subsection 1.2.7 are spherically stable, $\Sigma_{a}(\mathbb{R}) \simeq\left(S^{1}, \mathrm{pt}\right)$, and, for the twist, we have the relation $M[p](q)=$ $M[p-q] ; \Sigma_{a}(\mathbb{C}) \simeq\left(S^{2}, \mathrm{pt}\right)$ and $M[p](q)=M[p] ; \Sigma_{a}(\mathbb{H}) \simeq\left(S^{4}, \mathrm{pt}\right)$ and $M[p](q)=$ $M[p+2 q]$.

Now, by default, we assume that $A$ is a spherically stable theory.

1.6. $A$-groups. Since the Hopf algebra structure on $A\left(\mathbf{P}^{\infty}\right)$ used below in the oriented case is related to general homotopy properties of $\mathbf{P}^{\infty}$, it makes sense to discuss it before introducing orientations.

1.6.1. Definition. A smooth variety $G$ is called an $A$-group if $G$ is flat, the functor $X \rightarrow A(X \times G)$ on the category of smooth varieties is equipped with a Hopf algebra structure over $A(X)$, and the counit can be realized by a point pt $\rightarrow G$. An $A$-group $G$ is commutative if the multiplication in $A(X \times G)$ is commutative. This definition is also applicable to infinite-dimensional varieties.

If the space $\mathbf{P}^{\infty}$ is flat, it is equipped with the structure of an $A$-group, because the homotopy $X$-points of $\mathbf{P}^{\infty}$ form the group Pic $X$. Technically, the group structure is determined by the characteristic operators representing the geometry at the level of $A$. The properties of these operators imply the following statement (see Subsection 4.4.3).

1.6.2. Theorem. If the space $\mathbf{P}^{\infty}$ is flat, it is a commutative A-group with respect to the following data: the comultiplication $\gamma=j_{L \bowtie L}^{*}$, the antipode $i=j_{L^{-1}}^{*}$, where $L / \mathbf{P}^{\infty}$ is the tautological bundle, and the counit $\varepsilon=j_{1}^{*}$, where $\mathbf{1}=\mathcal{O}_{\mathrm{pt}}$.

For example, $\mathbf{P}^{n}$ and $\mathbf{P}^{\infty}$ are flat for an oriented theory (see Subsection 2.7). If $p A=0$ for a prime $p$ and $n=p^{i}-1$, then $\mathbf{P}^{n}$ is a subgroup of $\mathbf{P}^{\infty}$. This subgroup is the kernel of the $i$ th power of the Frobenius mapping. 
1.6.3. Remark. $A_{n}$ acts trivially on $\left(G_{+}\right)^{\otimes n}$ for a homotopy Abelian group, and $G_{+}$ can be used as a suspension preserving the $\otimes$-structure. Indeed, if for $f, g: X \rightarrow G$ the difference $f-g$ is null-homotopic, then $f^{*}=g^{*}$. Therefore, $(x, y) \simeq(-y,-x)$ and $(x, y, z) \simeq(-y,-x, z) \simeq(-y,-z, x) \simeq(z, y, x)$.

\section{§2. ORIENTED THEORIES}

In topology, an orientation of a cohomology theory is understood as an indication of certain data enabling one to choose compatible fundamental classes (orientations) of the fibers of the spherical bundles related to complex vector bundles. The definition of an orientation in terms of absolute spaces rather than bundles involves, instead of spherical bundles, their direct images in the category of spaces over a point, i.e., the Thom spaces.

2.1. Thom spaces. In topology, the Thom spaces are defined by contraction of the $\infty$-section of the fiberwise one-point compactification. For the reasons indicated in Subsection 1.5.1, in the algebraic situation, a different approach is used (see [2]), which turns out to be not less geometric.

2.1.1. Definition. The Thom space $T_{S} E$ of a vector bundle $E / X$ with support $S \subset X$ is the pair $(E, E-S)$, where $S$ is embedded into $E$ by the zero section.

We have $\operatorname{Th}_{S} E \otimes_{X} \operatorname{Th}_{S} F=\operatorname{Th}_{S}(E+F)$. A mapping $g: F / X \rightarrow E / Y$ of vector bundles that covers a mapping $f: X \rightarrow Y$ of smooth varieties is injective on fibers, is $S$-proper for a support $S \subset X$, and induces a mapping of Thom spaces $\operatorname{Th}_{S} F \rightarrow \mathrm{Th}_{T} E$ for each support $T \subset Y$ containing $f(S)$.

2.2. Orientations. In the next definition, it is useful to bear in mind that $A(\operatorname{Th} E)=$ $A_{X}(E)$, where $X$ is embedded into $E$ by the zero section $z$.

2.2.1. Definition. An orientation $\theta$ of a theory $A$ is a correspondence that, to each variety $X$ and each vector bundle $E / X$, assigns a two-sided $A(X)$-operator $\theta^{E}: A(X) \rightarrow$ $A_{X}(E)$ of degree $(\mathrm{rk} E)[2 \mathrm{rk} E]$ so that the following conditions are satisfied:

1) functoriality: $\tilde{f}^{*} \circ \theta^{E}=\theta^{F} \circ f^{*}$ for each specialization (see Subsection 4.3.4) $f: Y \rightarrow X, \tilde{f}: F \rightarrow E$ of vector bundles $E / X$ and $F / Y$;

2) multiplicativity: $\theta^{E+F}=q^{*} \theta^{E} \cdot p^{*} \theta^{F}$ for vector bundles $p: E \rightarrow X$ and $q: F \rightarrow$ $X$

3) nondegeneracy: all operators $\theta^{E}$ are isomorphisms.

A theory is orientable if such a $\theta$ exists, and is oriented if $\theta$ is fixed; $\theta^{E}$ is called the Thom operator and is denoted by $\theta$. An orientation is normalized if the Thom operator $\theta^{\mathbf{1}}$ coincides with the suspension isomorphism ( $\mathbf{1}$ is the standard one-dimensional bundle over a point, and, by definition, Th $\mathbf{1}=\Sigma_{a}$ ).

Below, some explanations concerning the notion of orientation are given and some properties of an orientable theory are presented.

2.2.2. Thom operators with supports. For an orientable theory, there exists a unique system of Thom operators $\theta_{S}^{E}: A_{S}(X) \rightarrow A\left(\operatorname{Th}_{S} E\right)$ defined for all triples $X, E, S$, where $S$ is a support in a smooth variety $X$ and $E / X$ is a vector bundle for which $\theta_{X}^{E}=\theta^{E}$, and the projection formula is valid (which describes the interaction with multiplication); 
i.e., all diagrams

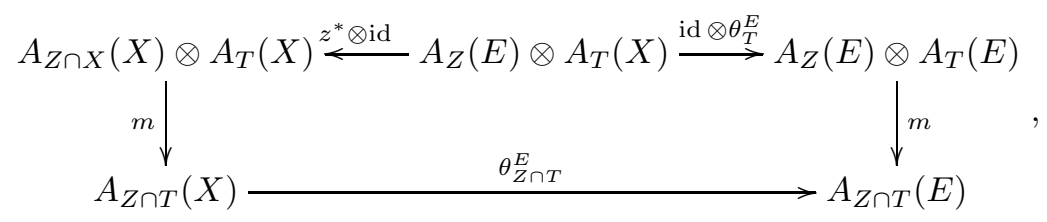

are commutative, where $Z$ is a support in $E, z: X \rightarrow E$ is the zero section, and $m$ is the multiplication. The uniqueness of $\theta_{S}^{E}$ follows from (12) for $T=X$ and $Z=p^{-1} S$, where $p: E \rightarrow X$ is the structure projection. Indeed, in this case, the composition $m \circ\left(z^{*} \otimes \mathrm{id}\right)$ has the section $\left(p^{*} \otimes\right.$ id) $\circ($ id $\otimes 1)$, whence $\theta_{S}^{E}=\theta^{E}(1) \cdot p_{S}^{*}$, where $p_{S}$ is the projection $\left(E, E-p^{-1} S\right) \rightarrow(X, X-S)$. The same formula proves existence. Namely, for the operators defined in this way, diagram (12) is commutative since both paths along the arrows of the diagram coincide with the composition $A_{Z}(E) \otimes A_{T}(X)=R \otimes A_{Z}(E) \otimes$ $A_{T}(X) \stackrel{1 \otimes \mathrm{id} \otimes \mathrm{id}}{\longrightarrow} A(X) \otimes A_{Z}(E) \otimes A_{T}(X) \stackrel{\theta^{E} \otimes \mathrm{id} \otimes p^{*}}{\longrightarrow} A_{X}(E) \otimes A_{Z}(E) \otimes A_{p^{-1} T}(E) \stackrel{m}{\longrightarrow}$ $A_{Z \cap T}(E)$.

The Thom operators with supports satisfy the following properties:

1) functoriality: $\tilde{f}^{*} \circ \theta_{S}^{E}=\theta_{T}^{F} \circ f^{*}$ for each specialization $f: Y \rightarrow X, \tilde{f}: F \rightarrow E$ of vector bundles $E / X$ and $F / Y$, where $T=f^{-1}(S)$;

2) multiplicativity: for vector bundles $p: E \rightarrow X$ and $F / X$, the composition $A_{S}(X) \stackrel{\theta_{S}^{E}}{\longrightarrow} A_{S}(E) \stackrel{\theta_{S}^{p^{*}(F)}}{\longrightarrow} A_{S}(E \oplus F)$ coincides with the operator $\theta_{S}^{E \oplus F}$

3) nondegeneracy: all operators $\theta_{S}^{E}$ are isomorphisms.

Functoriality and multiplicativity are obvious. By the Mayer-Vietoris triangle 1.3.4. nondegeneracy reduces to the case where $E=X \times \mathbf{A}^{r}$, and by multiplicativity, to $r=1$. In this case, $\theta^{E}$ is invertible and differs from the suspension by the invertible element $A^{0,0}(\mathrm{pt})$. Therefore, $\theta_{S}^{E}$ also differs from the suspension by an invertible element. The fact that the suspension for $(X, X-S)$ is invertible implies that $\theta_{S}^{E}$ is invertible.

2.2.3. Since $A$ is orientable, the action of $\operatorname{Aut}(E)$ on $A\left(\operatorname{Th}_{S} E\right)$ is trivial for a vector bundle $E / X$ and a support $S \subset X$. Indeed, for $\varphi \in \operatorname{Aut}(E)$, we have $\varphi^{*} \theta=\theta$ by functoriality, so that $\varphi^{*}=\theta \circ \theta^{-1}=\mathrm{id}$. This is not true for an arbitrary theory: in the topological $\mathbb{R}$-theory (see Subsection 1.5.10) with $\mathbb{Z}$-coefficients, the action is nontrivial for $E=\mathbf{A}^{1} / \mathrm{pt}$.

2.2.4. Centrality of the algebraic suspension. For an orientable theory $A$, the action of the symmetry group $S_{n}$ on $C^{\otimes n}$ is trivial (in the general case, only the action of $A_{n}$ is trivial, see Subsection 1.5.4). In particular, $C$ is central; i.e., the permutation of the symmetry structure $C^{\otimes 2} \stackrel{t}{\rightarrow} C^{\otimes 2}$ is trivial (a similar fact was proved in [12, 3.2.3]). This follows from Subsection 2.2.3, because $C^{n}=A\left(\operatorname{Th} \mathbf{1}^{n}\right)$ by stability, and the permutation is induced by an automorphism of $\mathbf{1}^{n}$. Thus, the permutation is trivial also on $R(1)^{\otimes 2}$ because $R[1]$ enters $C$ twice. In particular, we have $\varepsilon=1$ for orientable theories with values in bigraded modules (see Subsection 1.4.1); i.e., the algebra $A^{\bullet}(X)$ is commutative.

2.2.5. For vector bundles $E / X$ and $F / X$, the following diagram:

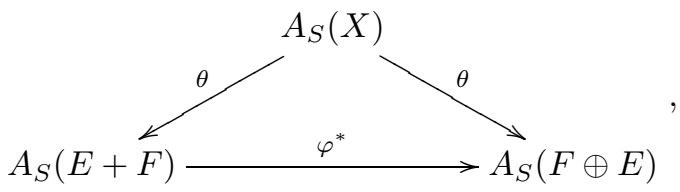


where $\varphi(f \oplus e)=e \oplus f$, is commutative. This is a consequence of functoriality.

2.2.6. Since the Thom operators are nondegenerate and multiplicative, we see that the Thom operator of the zero bundle is trivial, i.e., $\theta_{S}^{0}=\mathrm{id}$.

2.2.7. For a closed embedding $Z \subset Y$ of smooth varieties, a support $S \subset Z$, and a vector bundle $E / Y$, we have the commutative diagram

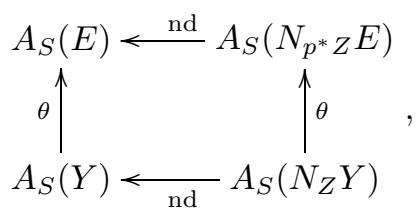

where $p: E \rightarrow Y$ is the structure projection. The structure of a vector $N_{Z} Y$-bundle on $N_{p^{*} Z} E$ is induced by the vector structure of $E / Y$ via the embedding $Z \rightarrow Y$; here we use the functoriality of the normal (see Subsection 4.2.1) and the fact that $Z$ and $p$ are transversal.

Diagram (14) is commutative because the Thom operators are natural and the deformation $D_{Z} E$ admits a natural structure of a vector bundle over $D_{Z} Y$ (see Subsection 4.2.8), the restriction of which to the typical fiber is $E / Y$ and the restriction to the special fiber is the bundle $N_{p^{*} Z} E$ over $N_{Z} Y$.

2.2.8. The deformation of a vector bundle $E / Z$ to the normal of the zero section preserves the Thom operators: the diagram

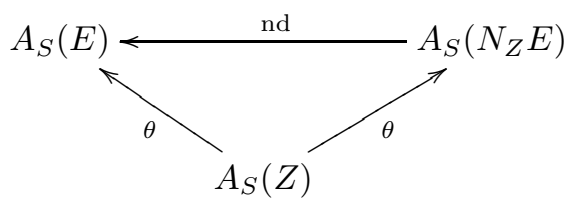

is commutative for every closed support $S \subset Z$. This is a special case of (14) (see Subsection 1.3.8).

2.2.9. For closed embeddings $Z \subset Y \subset X$ of smooth varieties and a support $S \subset Z$, the following diagram is commutative (this diagram plays a key role in the proof of the fact that the Gysin operators are natural; see Subsection 2.3.7):

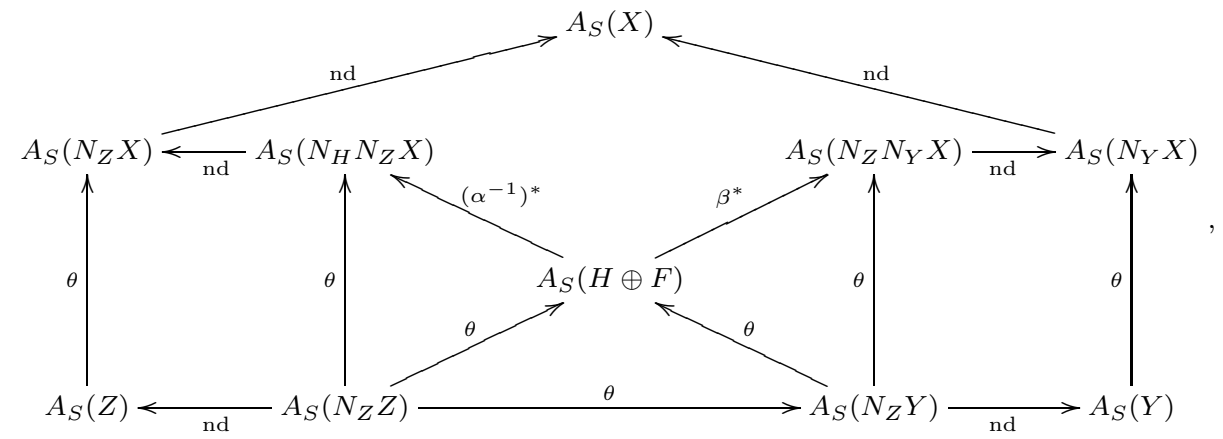

where $H=N_{Z} Y, F=N_{Z} X / N_{Z} Y$, and $\alpha$ and $\beta$ are the isomorphisms given in (39).

The diagram is commutative because the parts of it are commutative: the upper hexagon is diagram (9) in Subsection 1.3.13, the base squares are special cases of (14), the triangles adjacent to the squares are commutative by functoriality, and the central triangle is commutative because the Thom operators are multiplicative. 
2.2.10. Example: algebraic cobordism. The theory of algebraic cobordism is represented (see Subsection [1.2.6) by the spectrum MGL (see [2, 6.3]), where $\mathbf{M G L}(n)=$ $\operatorname{Th}\left(T_{n}\right), T_{n}$ is the tautological bundle on $\mathbf{G r}_{n}$, and the structure morphism $\Sigma_{a} \otimes \mathbf{M G L}(n)$ $\rightarrow \mathbf{M G L}(n+1)$ is given by the mapping $\mathbf{A}^{1} \times T_{n} \rightarrow T_{1+n},(t, e) \mapsto\left(t e_{1}+\operatorname{ver}(e)\right)$, where ver : $\left(x_{1}, x_{2}, \ldots\right) \mapsto\left(0, x_{1}, x_{2}, \ldots\right)$ is the shift in $\mathbf{A}^{\infty}$. The mappings $\mathbf{M G L}(n) \otimes$ $\operatorname{MGL}(m) \rightarrow \mathbf{M G L}(m+n),(x, y) \mapsto\left(x, \operatorname{ver}^{n} y\right)$, and $\Sigma_{a}^{n} \rightarrow \mathbf{M G L}(n),(x, y) \mapsto\left(x, \operatorname{ver}^{n} y\right)$, make MGL a commutative monoid in $\mathcal{S H}$. (see Subsection 1.2.6). This can be verified by using symmetric spectra (see [13, 2.5.5]).

There is a tautological orientation of MGL; for a bundle $E / X, \operatorname{rk} E=n$, it is given by the class $\theta^{E}(1) \in \mathbf{M G L}^{2 n, n}$ (Th $\left.E\right)$. Constructing this orientation, we may assume (see Subsection 4.4.2) that $E=f^{*} T_{n}$ for some mapping $f: X \rightarrow \mathbf{G r}_{n}$. In this case, $f$ induces a mapping Th $E \rightarrow \operatorname{Th} T_{n}$, i.e., an element $\theta(1) \in \operatorname{MGL}(\operatorname{Th} E)$.

2.2.11. The functorial $A(X)$-linear isomorphisms $A\left(\operatorname{Th} \mathbf{1}_{X}^{\mathrm{rk} E}\right) \rightarrow A(\operatorname{Th} E)$ form a $\mathbf{G}_{m}$-torsor $\operatorname{Det}_{A}(E)$. Taking the suspension isomorphism into account, we see that to choose an orientation of $E$ is the same as to choose an isomorphism $A\left(\operatorname{Th} 1_{X}^{\mathrm{rk} E}\right) \rightarrow A(\operatorname{Th} E)$, i.e., a trivialization of $\operatorname{Det}_{A}(E)$.

2.3. Gysin operators. For an oriented theory $A$, we introduce the Gysin operators, which are nonlinear versions of the Thom operators.

2.3.1. For an embedding $i: Y \rightarrow X$ of smooth varieties that is proper on a support $T \subset Y$, and for a support $S \supset f(T)$, the Gysin operator (of degree $[2 d](d)$, where $d=\operatorname{dim} X-\operatorname{dim} Y)$ is defined as the composition

$$
i_{\text {gys }}^{T, S}: A_{T}(Y) \stackrel{\theta}{\rightarrow} A_{T}\left(N_{Y} X\right) \stackrel{\text { nd }}{\longrightarrow} A_{T}(X) \stackrel{\text { s.e. }}{\longrightarrow} A_{S}(X),
$$

where nd is the operator of deformation to the normal (see Subsection 1.3.7) and $\theta$ is the Thom operator $\theta_{T}^{N_{Y} X}$.

Below, for brevity, we write $i_{\text {gys }}^{T}$ for $i_{\text {gys }}^{T, T}, i_{\text {gys }}$ for $i_{\text {gys }}^{Y, X}$, and $i_{\text {gys }}$ for $i_{\text {gys }}^{T, S}$ if $T$ and $S$ are clear. We consider the properties of Gysin operators.

2.3.2. The Gysin operator of the identity embedding is the identity. Moreover, for an isomorphism $i: Y \rightarrow X$ and a support $T \subset Y$, the operator $i_{\text {gys }}: A_{T}(Y) \rightarrow A_{S}(X)$, where $S=i(T)$, is inverse to $i^{*}$. This follows from Subsection 1.3.8.

2.3.3. The Gysin operator is a two-sided $A(X)$-operator (an $A(X)$-module structure on $A_{S}(X)$ was defined in Subsection 1.4.4, on $A_{T}(Y)$, this structure is induced by $i^{*}$ from the $A(Y)$-structure).

2.3.4. The Gysin operator $i_{\text {gys }}^{T}$ coincides with the Thom operator $\theta_{T}^{X}$ in the case where $X$ is a vector bundle on $Y$ and $Y$ is embedded by the zero section (see Subsection 2.2.8).

2.3.5. The Gysin operator $i_{\text {gys }}^{T, S}$ is an isomorphism for $T=S$ (because the Thom operators and the operator of deformation to the normal are invertible).

2.3.6. A Gysin operator is natural with respect to a transversal change of base, i.e., for a mapping $\tilde{X} \stackrel{f}{\rightarrow} X$ transversal to $Y$ (see Subsection 4.2.1), we have the commutative diagram

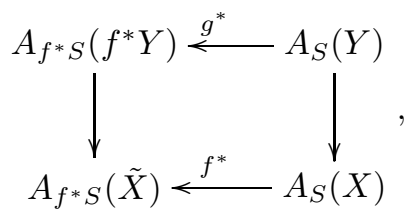


where $g=f \times_{X} Y$ and the vertical arrows are Gysin operators. This follows from the fact that deformation to the normal is natural with respect to a transversal change of the base (see Subsection 4.2.6).

2.3.7. For smooth embeddings $Z \subset Y \subset X$ and supports $S \subset Z, T \subset Y$, and $W \subset X$ such that $S \subset T \subset W, S$ is closed in $Y$ and in $X$, and $T$ is closed in $X$, we deduce that the composition of Gysin operators $A_{S}(Z) \rightarrow A_{T}(Y) \rightarrow A_{W}(X)$ coincides with the Gysin operator $A_{S}(Z) \rightarrow A_{W}(X)$.

Since the support extension operators are functorial, the statement reduces to the case where $S=T=W$, in which it means that the diagram in Subsection 2.2.9] is commutative: the operator $A_{S}(Z) \rightarrow A_{S}(X)$ is determined by the path from the left lower corner to the upper one along the left contour, and the composition by the path that first goes along the lower contour and then along the right contour.

2.3.8. For a closed embedding $i: Y \subset X$ of smooth varieties, the triangle

$$
A(Y) \stackrel{\text { s.e.o } i_{\text {gys }}^{Y}}{\longrightarrow} A(X) \rightarrow A(U) \stackrel{\left(i_{\text {gys }}^{Y}\right)^{-1} \circ \partial}{\longrightarrow} A(Y)[1]
$$

obtained from the triangle of the pair by changing the relative cohomology with the help of the Gysin operator, is exact and is called the Gysin triangle of the embedding $Y \subset X$.

2.3.9. Projection formula for $i_{\text {gys }}$. For an embedding $i: Y \rightarrow X$ of smooth varieties and supports $S, T \subset Y$ and $Z \subset X$ such that $S \subset Z$, we have the commutative diagram

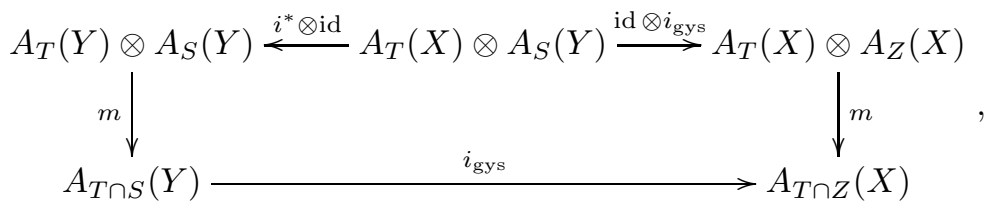

where $m$ is the multiplication. The proof reduces to the case where $Z=S$, because the Gysin operators $A_{S}(Y) \rightarrow A_{Z}(X)$ and $A_{T \cap S}(Y) \rightarrow A_{W \cap Z}(X)$ factor through the operators $A_{S}(Y) \rightarrow A_{S}(X)$ and $A_{T \cap S}(Y) \rightarrow A_{W \cap S}(X)$. In this case, the statement is proved by comparing the diagram with a similar diagram for the Thom operators (12) and recalling Subsections 1.3.9 and 1.4.5.

2.3.10. We have $h(y, 0)_{\text {gys }}=h(y, 1)_{\text {gys }}$ for a smooth embedding $h: Y \times \mathbf{A}^{1} \rightarrow X \times \mathbf{A}^{1}$ over $\mathbf{A}^{1}$. This follows from homotopy invariance and Subsection 2.3.6,

2.3.11. For a linear embedding $i: Q \rightarrow P$ of projective spaces, the operator $i_{\mathrm{gys}}$ : $A(Q) \rightarrow A(P)$ is a split monomorphism. If $\operatorname{codim} Q=1$, this follows from Subsection 1.3.15. The general case reduces to this by Subsection 2.3.7.

2.3.12. For a linear embedding $i: Q \rightarrow P$ of projective spaces, the operator $i_{\text {gys }}$ does not depend on $i$. This follows from Subsections 4.4.5] and 2.3.10, and from the fact that $i_{\text {gys }}$ is injective (see Subsection 2.3.11).

2.3.13. For a linear embedding $i: Q \rightarrow P$ of projective spaces, the operator $i^{*}$ does not depend on $i$ (for a nonorientable theory, generally, this is true only if $\operatorname{dim} Q<\operatorname{dim} P$ and even stronger restrictions are required for this to be true for a general change of the base). This follows from Subsection 1.3.15, the fact that $i_{\text {gys }}$ is injective (see Subsection 2.3.11) and that $i_{\text {gys }}$ (see Subsection 2.3.12) does not depend on a linear embedding.

We note that the automorphisms of $\mathbf{G}_{m}$ act nontrivially in cohomology even in the case of an orientable theory. For example, the involution $x \mapsto-x$ is nontrivial for the topological $\mathbb{R}$-theory with $\mathbb{Z} / 2$-coefficients (see Subsection 1.5.10). 
2.4. Parameters. We show (see Subsection 2.6.1) that any orientation can be constructed by quite a small set of data, namely, by a parameter. ${ }^{1}$

2.4.1. Definition. An operator $t: C \rightarrow A\left(\mathbf{P}^{\infty}\right)$, i.e., $t \in A^{2,1}\left(\mathbf{P}^{\infty}\right)$, is called a parameter if $t_{0}=0$ and $t_{1}$ is an isomorphism, where $t_{0}$ and $t_{1}$ are the components of $t \mid \mathbf{P}^{1}$ in the decomposition $A\left(\mathbf{P}^{1}\right)=R+C$ (see (11)). A parameter $t$ is normalized if $t_{1}=1$.

From now on, let $u \mid \mathbf{P}^{n}$ be the restriction $i^{*} u \in A^{2 d, d}\left(\mathbf{P}^{n}\right)$ for $u \in A^{2 d, d}\left(\mathbf{P}^{\infty}\right)$, which does not depend on the embedding $i: \mathbf{P}^{n} \rightarrow \mathbf{P}^{\infty}$ (see Subsection 4.4.3).

2.4.2. For a parameter $t$ and a vector bundle $E / X$, let $c=j_{L}^{*} t \in A^{1}(\mathbf{P} E)$, where $j_{L}^{*}: A\left(\mathbf{P}^{\infty}\right) \rightarrow A(\mathbf{P} E)$ is the characteristic operator of the tautological line bundle $L / \mathbf{P} E$ (see Subsection 4.4.3). We claim that $c$ induces an isomorphism

$$
\left[R+C+\cdots+C^{n}\right] \otimes A(X) \stackrel{1, c, \ldots, c^{n}}{\longrightarrow} A(\mathbf{P} E) \quad(n=\mathrm{rk} E-1) .
$$

Using the Mayer-Vietoris triangle (Subsection 1.3.4) and the fact that $j_{L}^{*}$ is natural (Subsection 4.4.3), we see that it suffices to prove the statement in the case where $E$ is trivial. The further arguments are performed over $X$ without mentioning this. Let $M_{i}=R+C+\cdots+C^{i}$, and let the operator $\gamma_{i}: M_{i} \rightarrow A\left(\mathbf{P}^{i}\right)$ be determined by the set $1, c, \ldots, c^{i}$. The restriction of $\gamma_{n}$ to $M_{n-1} \subset M_{n}$ is split by the composition of the restriction $A\left(\mathbf{P}^{n}\right) \rightarrow A\left(\mathbf{P}^{n-1}\right)$ and of $\gamma_{n-1}^{-1}: A\left(\mathbf{P}^{n-1}\right) \rightarrow M_{n-1}\left(\gamma_{n-1}\right.$ is invertible by induction). Therefore, $\gamma_{n} M_{n-1}$ is a direct summand of $A\left(\mathbf{P}^{n}\right)$ and $A\left(\mathbf{P}^{n}\right)=\gamma_{n} M_{n-1}+$ s.e. $A_{Z}\left(\mathbf{P}^{n}\right)$, where $Z=\{(0, \ldots, 0)\} \subset \mathbf{A}^{n} \subset \mathbf{P}^{n}$. This can be seen from the triangle of the pair $\left(\mathbf{P}^{n}, \mathbf{P}^{n}-Z\right)$ and the identification $A\left(\mathbf{P}^{n}-Z\right) \simeq A\left(\mathbf{P}^{n-1}\right)$ induced by the linear projection $\mathbf{P}^{n}-Z \rightarrow \mathbf{P}^{n-1}$.

We finish the proof if we verify that $c^{n}: C^{n} \rightarrow A\left(\mathbf{P}^{n}\right)$ factors through some isomorphism $u: C^{n} \rightarrow A_{Z}\left(\mathbf{P}^{n}\right)$. Let $H_{1}, \ldots, H_{n} \subset \mathbf{P}^{n}$ be the standard hyperplanes $\left(H_{1} \cap \cdots \cap H_{n}=Z\right)$, and $u$ the product $u_{1} \cdots u_{n}$, where $u_{i}: C \rightarrow A\left(\mathbf{P}^{n}, \mathbf{P}^{n}-H_{i}\right)$ are chosen so that $c: C \rightarrow A\left(\mathbf{P}^{n}\right)$ is a support extension for $u_{i}$, and therefore, $c^{n}$ is a support extension for $u$. The possibility of such a choice of $u_{i}$ is seen from the triangle of the pair $\left(\mathbf{P}^{n}, \mathbf{P}^{n}-H_{i}\right)$ and the fact that the restriction of $c$ to $\mathbf{P}^{n}-H_{i}$ is trivial because it is equal to $j_{M}^{*} t$, where $M=L \mid\left(\mathbf{P}^{n}-H_{i}\right)$ is trivial. Since the restriction $A_{Z}\left(\mathbf{P}^{n}\right) \rightarrow A_{Z}\left(\mathbf{A}^{n}\right)$ is invertible by excision, we see that $u: C^{n} \rightarrow A_{Z}\left(\mathbf{P}^{n}\right)$ is invertible if so is the mapping $v: C^{n} \rightarrow A_{Z}\left(\mathbf{A}^{n}\right)$ obtained by the composition of $u$ and the above restriction. Since the multiplication is functorial, we have $v=v_{1} \cdots v_{n}$, where $V_{i}=H_{i} \cap \mathbf{A}^{n}$ and $v_{i}: C \rightarrow A\left(\mathbf{A}^{n}, \mathbf{A}^{n}-V_{i}\right)$ is the restriction of $u_{i}$. Thus, it suffices to prove that $v_{i}$ and the multiplication $A\left(\mathbf{A}^{n}, \mathbf{A}^{n}-V_{1}\right) \otimes \cdots \otimes A\left(\mathbf{A}^{n}, \mathbf{A}^{n}-V_{n}\right) \rightarrow A_{Z}\left(\mathbf{A}^{n}\right)$ are invertible. Using the restriction to the $i$ th coordinate line $Z_{i}=\mathbf{A}^{1}$, we see that it suffices to prove the invertibility of the mappings $\left(v_{i} \mid Z_{i}\right): C \rightarrow A\left(Z_{i}, Z_{i}-0\right)$ and $A\left(Z_{1}, Z_{1}-0\right) \otimes \cdots \otimes A\left(Z_{n}, Z_{n}-0\right) \rightarrow A_{Z}\left(\mathbf{A}^{n}\right)$. The outer multiplication is invertible because it coincides with the suspension (see Subsection [1.5).

By excision, the invertibility of $\left(v_{i} \mid Z_{i}\right)$ follows from the invertibility of $\left(u_{i} \mid \mathbf{P}^{1}\right): C \rightarrow$ $A\left(\mathbf{P}^{1}, \mathbf{P}^{1}-0\right)$, where $\mathbf{P}^{1}$ is the closure of $Z_{i}$ in $\mathbf{P}^{n}$. By the construction of $u_{i}$, the operator $c$ is obtained from $\left(u_{i} \mid \mathbf{P}^{1}\right)$ by a support extension, so that $c \mid \mathbf{P}^{1}$ is a support extension of $u_{i} \mid \mathbf{P}^{1}$. Thus, the invertibility of $u_{i} \mid \mathbf{P}^{1}$ follows from the construction of $c$ and the definition of a parameter.

2.4.3. Cohomology of a projective bundle. For a vector bundle $E / X$, a support $S \subset X$, and the projection $p: \mathbf{P} E \rightarrow X$, we have an isomorphism

$$
A(\mathbf{P} E) \otimes_{A(X)} A_{S}(X) \rightarrow A_{p^{*} S}(\mathbf{P} E)
$$

\footnotetext{
${ }^{1}$ In [12, the notion of an orientation of a spectrum (by the choice of a parameter) was introduced, and the theorem on the cohomology of a projective bundle for representable theories was stated.
} 
given by multiplication. If we recall Subsection 2.4.2, we obtain the above statement by comparing the triangle of the pair $(X, X-S)$ tensored by $A(\mathbf{P} E)$ over $A(X)$ (which is well defined and exact because $A(\mathbf{P} E)$ is free, see Subsection 1.5.5) with the triangle of the pair $\left(\mathbf{P} E, \mathbf{P} E-p^{*} S\right)$.

2.4.4. Splitting principle. For a theory with parameter $t$ and a bundle $E / X$, there exists a smooth variety $\tilde{X}$ and a mapping $f: \tilde{X} \rightarrow X$ such that the operator $f^{*}$ : $A(X) \rightarrow A(\tilde{X})$ is injective and $f^{*} E$ is a sum of line bundles. Indeed, the operator $p^{*}: A(X) \rightarrow A(\mathbf{P} E)$, where $p: \mathbf{P} E \rightarrow X$ is a projection, is injective by Subsection 2.4.2, and the tautological bundle $L \subset p^{*} E$ splits off on a variety with the same cohomology (see Subsection 4.4.1). The proof is obtained by iterating this argument.

2.5. Characteristic classes. An oriented theory can be equipped with Chern classes. To this end, for technical reasons, it is convenient to use only the existence and the choice of a parameter (below, in Subsection 2.6.1, we establish a bijection between orientations and parameters).

2.5.1. The characteristic polynomial $\chi_{E}$. For a theory with parameter $t$ and a vector bundle $E$ on a smooth variety $X$, we consider the operator

$$
\operatorname{det}[t-u]: C^{n} \otimes \operatorname{Det}(\mathbf{P} \tilde{E}) \rightarrow \operatorname{Det}(\mathbf{P} \tilde{E}),
$$

where $n=\operatorname{rk} E, \tilde{X}=X \times \mathbf{P}^{\infty}, \tilde{E}=E \times \mathbf{P}^{\infty}$, and $u=j_{L}^{*}(t)$ for the tautological $L / \mathbf{P} E$.

In this formula, $\operatorname{Det}(\mathbf{P} \tilde{E})$ is the $n$th external power over $A(\tilde{X})$, i.e., the set of values of the universal skew-symmetric $A(\tilde{X})$-multilinear $n$-form on $A(\mathbf{P} \tilde{E})$. The external power exists since $A(\mathbf{P} \tilde{E})$ is "free" (see Subsections 1.5.5 and 2.4.2).

Since $A(\mathbf{P} E)$ is cyclic with respect to $u$, we see that $\operatorname{det}[t-u]=\chi_{E}(t)$, where $\chi_{E}(x)$ is a unique unitary polynomial of degree $n$ over $A^{\bullet}(X)$ for which $\chi_{E}(u)=0$.

For an exact sequence $0 \rightarrow E_{1} \rightarrow E \rightarrow E_{2} \rightarrow 0$ of vector bundles on a variety $X$, we have the relation $\chi_{E}=\chi_{1} \chi_{2}$, where $\chi_{i}=\chi_{E_{i}}$. In particular, $\chi_{E}=x^{n}$ for a trivial bundle $E$ of rank $n$.

Since $\chi_{1} \chi_{2}$ is a unitary polynomial of degree rk $E$, to prove the above relation it suffices to check that $\chi_{1}(u) \chi_{2}(u)=0$. The homotopy splitting (see Subsection 4.4.1) allows us to assume that $E=E_{1}+E_{2}$. In this case, the statement follows from Subsection 1.4.6. because the restriction of $\chi_{i}(u)$ to $A\left(\mathbf{P} E_{i}\right)$ is trivial.

2.5.2. Theorem. A theory with parameter $t$ can be equipped uniquely with Chern classes; i.e., to each vector bundle $E / X$ we can assign a set of operators $c_{i}(E) \in A^{2 i, i}(X)(i=$ $0,1, \ldots)$ satisfying the following conditions:

1) naturality: $c_{i}(E) \circ f^{*}=(\tilde{f})^{*} \circ c_{i}(F)$ for all $i$ and every specialization $f: Y \rightarrow X$, $\tilde{f}: F \rightarrow E$ of vector bundles $E / X$ and $F / Y$;

2) product formula: $c_{n}(E \oplus F)=c_{0}(E) c_{n}(F)+c_{1}(E) c_{n-1}(F)+\cdots+c_{n}(E) c_{0}(F)$;

3) normalization: $c_{0}\left(\mathcal{O}_{\mathbf{P}^{n}}(1)\right)=1, c_{1}\left(\mathcal{O}_{\mathbf{P}^{n}}(1)\right)=t \mid \mathbf{P}^{n}$, and $c_{i}\left(\mathcal{O}_{\mathbf{P}^{n}}(1)\right)=0$ for $i>1$.

Proof. Uniqueness follows from Corollary 2.4.4. Let $c_{i}(E)=a_{i}$, where $\chi_{E}(x)=x^{n}-$ $a_{1} x^{n-1}+\cdots+(-1)^{n} a_{n}$ is the characteristic polynomial (see Subsection 2.5.1). Naturality and normalization are valid tautologically. The product formula follows from the relation $\chi_{E}=\chi_{1} \chi_{2}$ (see Subsection 2.5.1).

Below, we present consequences of the basic properties of the Chern classes. 
2.5.3. For a trivial bundle, we have $c(E)=1$. The product formula reduces this statement to $E=\mathcal{O}$. In this case, the characteristic operator factors through a point, and the statement follows from normalization. Moreover, $c_{i}(E)=0$ for $i>\operatorname{rk} E$, and $c_{0}(E)=1$ for an arbitrary $E$. This follows from Subsection 2.4.4 and normalization.

2.5.4. For each exact sequence of vector bundles $0 \rightarrow E_{1} \rightarrow E \rightarrow E_{2} \rightarrow 0$, we have $c_{n}(E)=c_{0}\left(E_{1}\right) c_{n}\left(E_{2}\right)+\cdots+c_{n}\left(E_{1}\right) c_{0}\left(E_{2}\right)$. This follows from the product formula and Subsection 2.4.4.

2.5.5. For a vector bundle $E$ on a smooth variety $X$, the classes $c_{i}(E)$ are nilpotent for $i>0$. By Subsection 2.4.4, it suffices to prove this statement for line bundles, and, by universality (see Subsection 4.4.2), for $L=\mathcal{O}(-1)$ on $\mathbf{P}^{n}$. For a hyperplane $H \subset \mathbf{P}^{n}$, since the triangle of the pair $\left(\mathbf{P}^{n}, \mathbf{P}^{n}-H\right)$ is exact and $L \mid\left(\mathbf{P}^{n}-H\right)$ is trivial, there exists a $u \in A_{H}^{1}\left(\mathbf{P}^{n}\right)$ such that s.e. $(u)=c_{1}(L)$. Let $u_{0}, \ldots, u_{n}$ be similar elements for hyperplanes $H_{0}, \ldots, H_{n}$ in general position. Then $c_{1}(L)^{n+1}$ is 0 because it is an extension of the supports of $u_{0} \cdots u_{n} \in A_{H_{0} \cap \cdots \cap H_{n}}^{n+1}\left(\mathbf{P}^{n}\right)=A_{\varnothing}^{n+1}\left(\mathbf{P}^{n}\right)=0$.

2.5.6. Euler classes. The Euler class $e(E)$ of a vector bundle $E / X$ is the highest Chern class $c_{n}(E) \in A^{2 n, n}(X)$, where $n=\operatorname{rk} E$. In particular, $e\left(\mathcal{O}_{X}\right)=0$. We also have $e(E)=e\left(E_{1}\right) e\left(E_{2}\right)$ for an exact sequence of vector bundles $0 \rightarrow E_{1} \rightarrow E \rightarrow E_{2} \rightarrow 0$ (Corollary 2.5.4).

2.5.7. For a vector bundle $E$ on an infinite-dimensional variety $X=\lim _{\longrightarrow} X_{i}$ (see Subsection 4.3.4), the system $c_{j}\left(E_{i}\right) \in A^{2 j, j}\left(X_{i}\right)$ is compatible because the Chern classes are natural. Thus, the classes $c_{j}(E) \in A^{2 j, j}(X)$ and the Euler class are defined. The fact that the Chern classes on varieties are nilpotent (see Subsection 2.5.5) implies that the Chern classes on infinite-dimensional varieties are topologically nilpotent (a homogeneous element $v$ of a graded topological ring $S$ is said to be topologically nilpotent if the intersection of all $v^{i} S_{n-i}$ is zero for every $n$ ).

2.5.8. If we have an embedding $\mathcal{O}_{X} \rightarrow E$ or an epimorphism $E \rightarrow \mathcal{O}_{X}$ of vector bundles (see Subsection 2.5.6), then $e(E)=0$. In particular, $e\left(p^{*} E \otimes \mathcal{O}_{\mathbf{P} E}(1)\right)=0$ and $e\left(p^{*} E^{\vee} \otimes \mathcal{O}_{\mathbf{P} E}(-1)\right)=0$, where $p$ is the projection $\mathbf{P} E \rightarrow X$.

2.5.9. In a theory with parameter, the restriction $A(\mathbf{P} V) \rightarrow A(\mathbf{P} E)$ is a split epimorphism for bundles $E \subset V$. This follows from Subsection 2.4.2.

2.5.10. In a theory with parameter, we have the following split exact sequence for bundles $E, F, V=E+F$, and $N=N_{\mathbf{P} F} \mathbf{P} E: 0 \rightarrow A_{\mathbf{P} F}(N) \stackrel{\text { s.e.ond }}{\longrightarrow} A(\mathbf{P} V) \stackrel{i^{*}}{\longrightarrow}$ $A(\mathbf{P} E) \rightarrow 0$. This follows from the fact that (8) is exact (see Subsection 1.3.12) and the epimorphism $i^{*}$ is split (see Subsection 2.5.9).

2.5.11. For a theory with parameter $t$, a smooth variety $X$, and a vector bundle $V / X$ of rank $n$, the operator $\varepsilon=e\left(\mathcal{O}_{\mathbf{P} \infty}(1) \otimes V\right)-u^{n}$ is tensor nilpotent over $A(X)$, i.e., the operator $\varepsilon^{\otimes d}: C^{\text {nd }} \rightarrow A\left(\mathbf{P}_{X}^{\infty}\right) \otimes_{A(X)} \cdots \otimes_{A(X)} A\left(\mathbf{P}_{X}^{\infty}\right)$ is trivial for sufficiently large $d$. Here, $\mathbf{P}_{X}^{\infty}=\mathbf{P}^{\infty} \times X$ and $u=t \otimes 1$, where $\nabla=p_{1}^{*} \otimes p_{2}^{*}$ for the projections $p_{1}$ and $p_{2}$ to the factors of $\mathbf{P}^{\infty} \times X$, and the operation $\otimes_{A(X)}$ (see Subsection 1.5.5) is well defined (see Subsection 2.4.2).

This follows from the fact that, in the expansion of $e\left(\mathcal{O}_{\mathbf{P} \infty}(1) \otimes V\right)-u^{n}=a_{n}+$ $a_{n-1} u+\cdots$ in powers of $u$, the coefficients are uniformly nilpotent (all products of $a_{i}$ in a number greater than a certain bound are zero) because, by $A(X)$-linearity, the coefficients of each monomial in the expansion of $\varepsilon^{\otimes d}$ can be collected in one factor. The fact that the coefficients are uniformly nilpotent follows from the existence of polynomials $p_{n}, p_{n-1}, \ldots$ over $R^{\bullet}$ such that $p_{i}(0)=0$ and $a_{i}=p_{i}\left(c_{1}(V), \ldots, c_{n}(V)\right)$. For a line bundle 
$V$, existence reduces to the case where $X=\mathbf{P}^{N}$ and $V=\mathcal{O}(1)$ by universality, where the elements of $A^{\bullet}(X)$ are polynomials in $c_{1}(V)$. The values $p_{i}(0)$ are seen from the restriction to $\mathbf{P}^{1}$ and the normalization of $c_{1}$. The general case can be reduced to the case of a line bundle by Subsection 2.5.6 and the homotopy splitting (Subsection 4.4.1).

2.5.12. For a theory with parameter $t$, a smooth variety $X$, and a vector bundle $V / X$ of rank $n$, we have the following split exact sequence:

$$
0 \rightarrow C^{n} \otimes A\left(\mathbf{P}_{X}^{\infty}\right) \stackrel{u}{\rightarrow} A\left(\mathbf{P}_{X}^{\infty}\right) \stackrel{f}{\rightarrow} A(\mathbf{P} V) \rightarrow 0,
$$

where $f=m \circ\left(j_{L}^{*} \otimes p^{*}\right)$ under the identification $A\left(\mathbf{P}_{X}^{\infty}\right) \simeq A\left(\mathbf{P}^{\infty}\right) \otimes A(X), L=\mathcal{O}_{\mathbf{P} V}(-1)$, $j_{L}^{*}$ is the characteristic operator (4.4.3), $p: \mathbf{P} V \rightarrow X$ is the structure projection, $m$ is the multiplication, and $u$ is the multiplication by the Euler class $e=e\left(\mathcal{O}_{\mathbf{P} \infty}(1) \otimes V\right) \in$ $A^{n}\left(\mathbf{P}_{X}^{\infty}\right)$ (see Subsection 1.5.5).

Indeed, the expansion of $A\left(\mathbf{P}_{X}^{\infty}\right)=M_{0} \oplus M_{1}$, where $M_{0}=\left(C^{0}+\cdots+C^{n-1}\right) \otimes$ $A(X), M_{1}=C^{n} \otimes A\left(\mathbf{P}_{X}^{\infty}\right)$, in powers of the parameter (see Subsection 2.4.2) induces decompositions $u=u_{0} \times u_{1}, e=e_{0} \times e_{1}$, and $f=f_{0} \oplus f_{1}$. In particular, $e_{1}=\operatorname{pr} \circ$ $e: C^{n} \rightarrow M_{1}$, where pr is the projection to $C^{n} \otimes A\left(\mathbf{P}_{X}^{\infty}\right)=M_{1}$ in the expansion of $A\left(\mathbf{P}_{X}^{\infty}\right)=M_{0} \oplus M_{1}$. Here $u_{1}$ is multiplication by the class $e_{1}: C^{n} \rightarrow M_{1}$. The fact that (20) is exact and splits follows from the invertibility of $u_{1}, f_{0}$ (see below), the equation $f_{0} u_{0}+f_{1} u_{1}=0$ (implied by Subsection 2.5.8), and the fact that the following sequences, where the lower sequence is exact and split, are isomorphic:

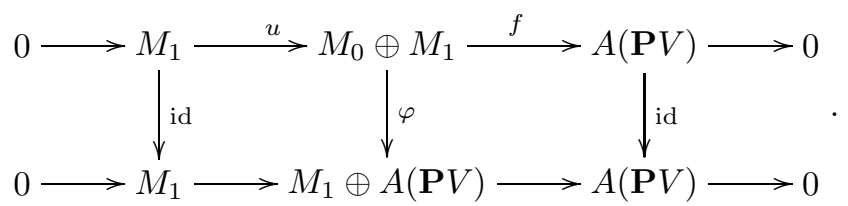

Here $\varphi=\left(u_{1}^{-1} \circ \operatorname{pr}\right) \times f$ and $\varphi^{-1}=\left(-f_{0}^{-1} f_{1} \oplus f_{0}^{-1}\right) \times\left(u_{1} \oplus 0\right)$. The invertibility of $f_{0}$ is a consequence of Subsection 2.4.2 and $u_{1}$ is invertible because id $-u_{1}$ is nilpotent. To prove nilpotency, we note that the operator $\phi: A\left(\mathbf{P}_{X}^{\infty}\right) \rightarrow A\left(\mathbf{P}_{X}^{\infty}\right), \phi=\left(\right.$ id $\left.-u_{1}\right) \otimes C^{-n}$, coincides with multiplication by $\left(t^{n}-u_{1}\right) \otimes C^{-n}: R \rightarrow A\left(\mathbf{P}_{X}^{\infty}\right)$. Here, the $t$-expansion of $\left(t^{n}-u_{1}\right)$ has the form $a_{0} t^{n}+a_{1} t^{n+1}+\cdots$, where the $a_{i}$ are defined by the expansion $u-t^{n}=a_{n}+a_{n-1} t+\cdots$. It remains to use Subsection 2.5.11, because $\left(t^{n}-u_{1}\right)^{\otimes N}$ splits off from $\left(t^{n}-u\right)^{\otimes N}$.

2.5.13. Applying (20) to the tautological bundle $E=\mathcal{O}(-1)$ on $X=\mathbf{P}^{n}$ and passing to the limit, we obtain the split exact sequence

$$
0 \rightarrow C \otimes A\left(\mathbf{P}^{\infty} \times \mathbf{P}^{\infty}\right) \stackrel{e(\mathcal{O}(1) \bigotimes \mathcal{O}(-1))}{\longrightarrow} A\left(\mathbf{P}^{\infty} \times \mathbf{P}^{\infty}\right) \stackrel{\Delta^{*}}{\longrightarrow} A\left(\mathbf{P}^{\infty}\right) \rightarrow 0
$$

( $\Delta$ is the diagonal).

2.6. The space of orientations. An orientation $\theta$ and a parameter $t$ are compatible if $i_{\text {gys }}(1)=c_{1}\left(\mathcal{O}_{\mathbf{P}^{n}}(1)\right)$ for every $n>0$ and some linear embedding $i: \mathbf{P}^{n-1} \subset \mathbf{P}^{n}$, where $i_{\text {gys }}$ is determined by $\theta$ and $c_{1}$ is determined by $t$ (see Subsection 2.5.2).

2.6.1. Theorem. For every orientation $\theta$, there exists a unique parameter $t(\theta)$ compatible with $\theta$. For every $t$, there exists a unique orientation $\theta_{t}$ compatible with $t$. Thus, the orientations are identified with the parameters, and the normalized orientations are identified with the normalized parameters.

Proof. We prove the uniqueness of $t(\theta)=t$ by verifying that $t \mid \mathbf{P} V$ is uniquely determined by $\theta$ (this suffices because, by definition (see Subsection 4.3.1), we have $t=\lim (t \mid \mathbf{P} V$ ), where $V$ is taken from the inductive system defining $\mathbf{P}^{\infty}$; see Subsection 4.3 .3 . Let 
$i: \mathbf{P}^{n-1} \rightarrow \mathbf{P}^{n}$ be the embedding in the definition of compatibility, and let $\alpha: \mathbf{P}^{n} \rightarrow \mathbf{P} V$ be an arbitrary linear isomorphism. Then $t \mid \mathbf{P} V=\alpha_{\text {gys }} \alpha^{*}(t \mid \mathbf{P} V)=\alpha_{\text {gys }} i_{\text {gys }}(1)$, where the first equation follows from Subsection 2.3.2 and the second is a consequence of the relation $\alpha^{*}(t \mid \mathbf{P} V)=t \mid \mathbf{P}^{n}$ (see Subsection 2.4) and the compatibility of $t$ and $\theta$.

By the splitting principle (see Subsection 2.4.4) and multiplicativity, it suffices to prove that $\theta_{t}^{L}$ is unique for a line bundle $L$ and, by universality (see Subsection 4.4.2), for $L=\mathcal{O}_{\mathbf{P} E}(1)$, where $E$ is taken from the inductive system defining $\mathbf{P}^{\infty}$. Let $i: \mathbf{P} E \rightarrow \mathbf{P} V$, where $V$ is taken from the inductive system and $V=E+F$, where $\operatorname{dim} F=1$. Since the mapping $i$ is isomorphic to the embedding $\mathbf{P}^{n-1} \subset \mathbf{P}^{n}$, we see that $i_{\text {gys }}(1)=c_{1}(L)$, because $\theta_{t}$ and $t$ are compatible and the Gysin operators and Chern classes are natural. Therefore, $i_{\text {gys }}$ is determined by $t$. Since s.e. : $A_{\mathbf{P} E}(\mathbf{P} V) \rightarrow A(\mathbf{P} V)$ is injective (see Subsection 1.3.15), $i_{\mathrm{gys}}^{\mathbf{P E}}$ is also determined by $t$. Moreover, by excision, it follows that $u_{\text {gys }}^{\mathbf{P E}}$ is also determined by $t$, where $u$ is the embedding $\mathbf{P} E \subset U=\mathbf{P} V-\mathbf{P} F$. Since the Gysin operators are functorial, we have $u_{\text {gys }}^{\mathbf{P} E}=(\varphi \circ z)_{\text {gys }}^{\mathbf{P} E}=\varphi_{\text {gys }}^{\mathbf{P} E} \circ z_{\text {gys }}^{\mathbf{P} E}$, where $z: \mathbf{P} E \rightarrow L$ is the zero section and $\varphi: L \rightarrow U$ is the isomorphism occurring in Subsection 4.4.8. Since $z_{\text {gys }}^{\mathbf{P E}}=\theta^{L}$ (see Subsection 2.3.4), we have $u_{\text {gys }}^{\mathbf{P E}}=\varphi_{\text {gys }}^{\mathbf{P E}} \circ \theta^{L}$, whence $u_{\text {gys }}^{\mathbf{P E}}=\left(\varphi^{-1}\right)^{*} \circ \theta^{L}$ (see Subsection 2.3.2). Thus, the operator $\theta^{L}$ is determined by $t$.

To an orientation $\theta$, we assign the parameter $t(\theta)=\lim _{V} t_{V}$, where $t_{V}=i_{\text {gys }}(1)$ for a hyperplane $i: \mathbf{P} E \rightarrow \mathbf{P} V$. Here, $t_{V}$ depends on $V$ but does not depend on $E$ (this follows from Subsection 2.3.12). For $f: \mathbf{P} W \subset \mathbf{P} V$, we choose a hyperplane $i: \mathbf{P} E \subset \mathbf{P} V$ in the general position with $\mathbf{P} W$ and consider the embedding $w: \mathbf{P} F \subset \mathbf{P} W$, where $F=W \cap E$. Then $f^{*} i_{\text {gys }}(1)=w_{\text {gys }}(1)$ by Subsection 2.3.6. Thus, $t_{V} \mid \mathbf{P} W=t_{W}$, and so the system $t_{V}$ is compatible and $t(\theta)$ is well defined. Moreover, we have $t(\theta) \mid \mathbf{P} V=t_{V}$, so that $t(\theta)$ is a parameter by 2.3.5. By construction, $t(\theta)$ is compatible with $\theta$.

Now, for a parameter $t$, we construct an orientation $\theta$ compatible with $t$. For every bundle $E / X$, we have the split exact sequence

$$
0 \rightarrow A_{X}(E) \stackrel{\text { s.e.ond }}{\longrightarrow} A(\mathbf{P} V) \stackrel{i^{*}}{\longrightarrow} A(\mathbf{P} E) \rightarrow 0,
$$

where $V=E+F, F$ is a trivial one-dimensional bundle, and $i: \mathbf{P} E \rightarrow \mathbf{P} V$ is the embedding obtained from the sequence in Subsection 2.5.10 after the identifications $E=N_{X}(\mathbf{P} V)$ (see Subsection 4.4.7) and $\mathbf{P} F=X$. Now, $i^{*} e\left(p^{*} E \otimes \mathcal{O}_{\mathbf{P} V}(1)\right)=0$ (see Subsection 2.5.8), where $p: \mathbf{P} V \rightarrow X$ is a projection, and (21) is exact; consequently, there is a unique $\theta^{E}(1) \in A_{X}(E)$ for which

$$
\text { s.e. } \circ \text { nd } \circ \theta^{E}(1)=e\left(p^{*} E \otimes \mathcal{O}_{\mathbf{P} V}(1)\right) \text {. }
$$

The operators $\theta_{E}$ determine an orientation. Indeed, naturality is obvious, and multiplicativity is fulfilled because it is fulfilled for the Euler classes. The Mayer-Vietoris triangle, naturality, and multiplicativity reduce the proof of nondegeneracy to the case where $E=1$. In this case, $\mathbf{P} V=\mathbf{P}^{1}$, and $e\left(p^{*} E \otimes \mathcal{O}_{\mathbf{P} V}(1)\right)=e\left(\mathcal{O}_{\mathbf{P} V}(1)\right)$ coincides with $t \mid \mathbf{P}^{1} \otimes A(X)$ and is nondegenerate by the definition of a parameter and the fact that the suspension is invertible.

To prove that $\theta$ and $t$ are compatible, we consider vector spaces $E \subset V$ with $V=E+F$, $\operatorname{dim} F=1$, an embedding $i: \mathbf{P} E \rightarrow \mathbf{P} V$, bundles $N=N_{\mathbf{P} E} \mathbf{P} V$ and $\tilde{N}=N+L$, where $L$ is a trivial one-dimensional bundle, and the mappings $\sigma: \mathbf{P} \tilde{N} \rightarrow \mathbf{P} V$ and $\pi: \mathbf{P} \tilde{N} \rightarrow \mathbf{P} E$ (see Subsection 4.4.9). By the isomorphism $\sigma^{*} \mathcal{O}_{\mathbf{P} V}(1) \simeq p^{*} N \otimes \mathcal{O}_{\mathbf{P} \tilde{N}}(1)$ (see Subsection 4.4.9), we obtain

$$
\sigma^{*} c_{1}\left(\mathcal{O}_{\mathbf{P} V}(1)\right)=c_{1}\left(p^{*} N \otimes \mathcal{O}_{\mathbf{P} \tilde{N}}(1)\right) .
$$

Here, $c_{1}\left(p^{*} N \otimes \mathcal{O}_{\mathbf{P} \tilde{N}}(1)\right)=s_{1} \circ$ ndo $\theta^{N}(1)$ by the construction of $\theta$, where nd : $A_{\mathbf{P} E}(N) \rightarrow$ $A_{\mathbf{P} E}(\mathbf{P} V)$ is the operator of deformation to the normal, and $s_{1}: A_{\mathbf{P} E}(\mathbf{P} \tilde{N}) \rightarrow A(\mathbf{P} \tilde{N})$ 
is a support extension. On the other hand, $\sigma^{*} c_{1}\left(\mathcal{O}_{\mathbf{P} V}(1)\right)=\sigma^{*} s_{2} \circ \operatorname{nd}(u)$, where $u \in$ $A_{\mathbf{P} E}^{2,1}(\mathbf{P} V)$, and $s_{2}: A_{\mathbf{P} E}(\mathbf{P} V) \rightarrow A(\mathbf{P} V)$ is a support extension. This follows from the fact that the sequence in Subsection 2.5 .10 is exact because $c_{1}\left(\mathcal{O}_{\mathbf{P} V}(1) \mid \mathbf{P} F\right)=0$. Thus, $s_{1} \circ \mathrm{nd} \circ \theta^{N}(1)=\sigma^{*} s_{2} \circ \operatorname{nd}(u)=s_{1} \circ \operatorname{nd}(u)$ (the first relation is a consequence of (22), and the second follows from the uniqueness of s.e. and nd). Since $s_{2} \circ$ nd is injective (see Subsection 2.5.10), we obtain $\operatorname{nd}(u)=\operatorname{nd} \circ \theta^{N}(1)$ and $i_{\text {gys }}(1)=\operatorname{nd} \circ \theta(1)=\operatorname{nd}(u)=$ $c_{1}\left(\mathcal{O}_{\mathbf{P} V}(1)\right)$, which proves that $\theta$ and $t$ are compatible. The proof of the statement concerning normalization is omitted.

2.6.2. Corollary. A theory is orientable if and only if the restriction $A^{2,1}\left(\mathbf{P}^{\infty}\right) \rightarrow$ $A^{2,1}\left(\mathbf{P}^{1}\right)$ is surjective for a linear embedding.

2.6.3. Étale cohomology. In Example 1.5.6, the parameter $t=\lim c_{1}\left(\mathcal{O}_{\mathbf{P} V}(1)\right)$, where

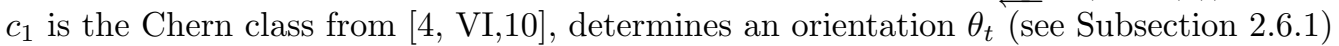
and Chern classes (see Subsection 2.5.2) coinciding with the classes from [4, VI,10] by uniqueness (see Subsection 2.5.2 and [4, VI,10.3]).

2.6.4. Motivic cohomology. In Example 1.5.8, the parameter $t=\lim t_{V}$ determines an orientation. Here $t_{V}$ is the class of $\mathcal{O}_{\mathbf{P} V}(1)$ under the composition

$$
\operatorname{Pic} \mathbf{P} V \rightarrow H^{2}(\mathbf{P} V, \mathbf{Z}(1)) \rightarrow H^{2}\left(\mathbf{P} V, F^{1}\right)
$$

(the first operator was given in [7, 3.2.1] and the second operator is a twist of the unity of $F$ ).

2.6.5. Topological theories (see Subsection 1.5 .10 ) . For the $\mathbb{R}$-theory, we have the parameter $t=\lim _{V}$, where $t_{V}=w_{1}(L(\mathbb{R})) \in A^{2}, 1(\mathbf{P} V)=H^{1}(\mathbf{P} V(\mathbb{R}), \mathbf{Z} / 2)$, and $L=\mathcal{O}_{\mathbf{P} V}(1)$. The corresponding Chern classes coincide with the Stiefel-Whitney classes of the real points of the bundle. For the $\mathbb{C}$-theory, the parameter is $t=\lim _{V} t_{V}$, where $t_{V}=$ $c_{1}(L(\mathbb{C})) \in A^{2,1}(\mathbf{P} V)=H^{2}(\mathbf{P} V(\mathbb{C}), \mathbf{Z})$, and the corresponding Chern classes coincide with the Chern classes of complex points of the bundle. For the topological $\mathbb{H}$-theory, we have the parameter $t=\lim t_{V}$, where $t_{V}=p_{1}^{s}(L(\mathbb{H})) \in A^{2,1}(\mathbf{P} V)=H^{4}(\mathbf{P} V(\mathbb{H}), \mathbf{Z})$, and the Chern classes coincide with the symplectic Pontryagin classes of the quaternionic points of the bundle.

In what follows, $A$ is equipped with an orientation $\theta$, and thus, with the Chern classes constructed by a parameter compatible with the orientation.

2.7. The formal group of an oriented theory. For an oriented theory $A$, we construct a commutative one-dimensional formal group. The orientation fixes a coordinate of this group, and, thus, a formal group law. The relationship between the group law and the Chern classes plays a significant role and is described below. Since it is useful to deal with two different orientations of the same theory, particular attention should be paid to the dependence of the structures on the choice of an orientation.

For an oriented theory $A$, the spaces $\mathbf{P}^{n}$ and $\mathbf{P}^{\infty}$ are $A$-flat (see Subsections 1.5 .2 and 2.4.2). Therefore, in the case of an oriented theory, the comultiplication $\gamma$, the antipode $i$, and the counit $\varepsilon$ (Subsection 1.6.2) determine the structure of a commutative $A$-group (1.6) on $\mathbf{P}^{\infty}$ (Subsection 1.6).

2.7.1. The formal group of $A$. The structure of an $A$-group on $\mathbf{P}^{\infty}$ induces the structure of a commutative $R^{\bullet}$-coalgebra on $S^{\bullet}=A^{\bullet}\left(\mathbf{P}^{\infty}\right)$. This structure is called a onedimensional formal group because $S^{\bullet}$ is a graded topological $R^{\bullet}$-algebra isomorphic to $R^{\bullet}[[t]]$ (the topology is defined on each homogeneous component), where the $n$-component of $R[[t]]$ is formed by the formal series $\sum a_{i} t^{i} \operatorname{such}$ that $\operatorname{deg}\left(a_{i}\right)=n-i$ with $t$-adic topology (where the system of neighborhoods of zero is $t^{i} R_{n-i}^{\bullet}$ ). Indeed, there is a natural 
isomorphism $R^{\bullet}[[u]] \rightarrow S^{\bullet}, u \mapsto t$, where $t$ is an arbitrary parameter (see Subsection 2.4.1).

2.7.2. The formal group law $\phi$. Every orientation of $A$ gives us the corresponding parameter $t$ (see Subsection 2.6.1), and therefore, the formal group law

$$
x++_{\phi} y=\phi(x, y)=\sum a_{i, j} x^{i} y^{j}=x+y+\cdots,
$$

expressing the image of $t$ under the comultiplication $\gamma$ in the form of a power series in $x=t \otimes 1$ and $y=1 \otimes t$. Moreover, there is a series $\iota(t)=-t+\cdots$ expressing the image of $t$ under the antipode, and the series $x-{ }_{\phi} y=x+{ }_{\phi} \iota(y)=x-y+\cdots$ for the difference in the formal group. For example, we have $\phi(x, y)=x+y$ for the étale (2.6.3), motivic (2.6.4), and topological theories (2.6.5) (for other examples, see Subsections 3.3 .2 and 3.3.3.

2.7.3. Points of a formal group and local parameters. For a smooth (possibly, infinite-dimensional) variety $X$, let $P(X)$ be the set of all $X$-points of $\mathbf{P}^{\infty}$ (with respect to $A)$, i.e., the set of homomorphisms of $A\left(X \times \mathbf{P}^{\infty}\right) \rightarrow A(X)$ of $A(X)$-algebras. This set does not depend either on the choice or on the existence of an orientation. The structure of an $A$-group on $A\left(\mathbf{P}^{\infty}\right)$ induces a group structure on $P(X)$; the mapping Pic $X \rightarrow P(X), L \mapsto j_{L}^{*}$ (see Subsection 4.4.3), is a group homomorphism and its zero is the standard $X$-point of $\mathbf{P}^{\infty}$ induced by the constant mapping $X \rightarrow \mathbf{P}^{\infty}$ (by Subsection 4.4.3. the latter can be arbitrary).

An affine parameter $u$ for $B=A\left(X \times \mathbf{P}^{\infty}\right)$ (see Subsection 4.5.1) is called a local parameter at a point $x \in P(X)$ if $x \circ u=0$. With an affine parameter $u$, we associate the point $x$ obtained by the composition of $\varphi_{u}^{-1}$ (see Subsection 4.5.1) with the projection to $A(X)$; here $u$ is a local parameter at $x$. The notion of a parameter (see Subsection 2.4.1) coincides with the notion of a local parameter over $X=$ pt at the standard point. Moreover, we have the set $F(X)=\left\{x \in A^{2,1}(X) \mid x\right.$ is topologically nilpotent $\}$ (see Subsection 4.3.2), which also depends neither on the choice of an orientation nor on its existence.

We have the identification $P(X) \simeq F(X), x \mapsto x \circ t$, which depends on $t$ (i.e., on the choice of an orientation). Indeed, by Subsections 2.4.2 and 2.5.1, the set of $A(X)$-points of $A\left(X \times \mathbf{P}^{n}\right)$ (the set of homomorphisms $A\left(X \times \mathbf{P}^{n}\right) \rightarrow A(X)$ of $A(X)$-algebras) is identified with the set of all $x$ in $A^{2,1}(X)$ such that $x^{n}=0$; therefore (see Subsection 4.3.1), we obtain $P(X)=\lim _{j} \lim _{i}\left\{x \in A^{2,1} 1\left(X_{j}\right) \mid x^{i}=0\right\}=\lim _{j}\left\{x \in A^{2,1}\left(X_{j}\right) \mid x\right.$ is nilpotent $\}=$ $F(X)$.

The identification $P(X) \simeq F(X)$ determines the structure of an Abelian group on $F(X)$, with the addition and the inverse element given by the series $\phi(x, y)$ and $\iota(x)$, respectively (see Subsection 2.7.2). The mapping $c_{1}: \operatorname{Pic} X \rightarrow F(X)$ (see Subsection 2.5.5) factors through the above homomorphism Pic $X \rightarrow P(X)$ and is a homomorphism to $F(X)$. This means that, for line bundles $L$ and $M$, the following relations are valid:

$$
\begin{aligned}
c_{1}(L \otimes M) & =c_{1}(L)+{ }_{\phi} c_{1}(M), \\
c_{1}\left(L \otimes M^{-1}\right) & =c_{1}(L)-{ }_{\phi} c_{1}(M), \\
c_{1}\left(L^{-1}\right) & =\iota\left(c_{1}(L)\right) .
\end{aligned}
$$

2.7.4. Invariant form. By definition, a differential form $\omega \in \Omega\left(S^{\bullet}\right)$ is invariant if $\gamma \omega=\omega \otimes 1+1 \otimes \omega$. The set $\Omega_{\text {inv }}$ of invariant forms is a graded free $R^{\bullet}$-module of rank one, and the restriction $\Omega_{\text {inv }} \rightarrow \Omega_{0}=I / I^{2}$, where $I$ is the kernel of the counit $S^{\bullet} \rightarrow R^{\bullet}$, is an isomorphism. Thus, for a parameter $t$, we have a unique form $\omega_{\text {inv }} \in \Omega_{\text {inv }}$ normalized 
by the condition that the restriction of $\omega_{\text {inv }}$ is $t \bmod I^{2}$, or, in other words, that the series

$$
\omega_{\text {inv }} / d t=1+a_{1} t+a_{2} t^{2}+\cdots
$$

starts with 1 . The invariance and normalization conditions imply that $\omega_{\text {inv }} / d t=1 / \phi_{x}^{\prime}(0, t)$ (e.g., for the étale, motivic, and topological theories we obtain $\omega_{\text {inv }}=d t$; see Subsections 3.3 .2 and 3.3 .3 for other examples). It follows that $\omega_{\text {inv }} / d t=\left[\left(x-{ }_{\phi} y\right) /(x-y)\right](t, t)$. In other words, for the formal law $\phi$ and the diagonal $\Delta: \mathbf{P}^{\infty} \rightarrow \mathbf{P}^{\infty} \times \mathbf{P}^{\infty}$ we have

$$
\omega_{\mathrm{inv}}=\Delta^{*}\left[\left(x-{ }_{\phi} y\right) /(x-y)\right] d t \text {. }
$$

\section{$\S 3$. Transfers}

As before, we assume that a theory $A$ in question is equipped with an orientation $\theta$, and, therefore, with Chern classes constructed by a parameter $t$ compatible with the orientation.

\subsection{The definition of transfers. Relationship with orientations.}

3.1.1. Definition. A theory $A$ is equipped with transfers if, for every mapping $f$ : $X \rightarrow Y$ of smooth varieties that is proper on a support $S \subset X$, and for every support $T$ containing $f(S)$, an operator of direct image $f_{!}: A_{S}(X) \rightarrow A_{T}(Y)$ of degree $(d)[2 d]$, where $d=\operatorname{dim} Y-\operatorname{dim} X$, is given so that the following conditions are fulfilled:

1) functoriality: $(f g)_{!}=f_{!} g_{!}, \mathrm{id}_{!}=\mathrm{id}$;

2) linearity in the base: $f_{!}^{S \cap S^{\prime}, T \cap T^{\prime}}\left(\right.$ id $\left.\cdot f^{*}\right)=f_{!}^{S, T} \cdot$ id as operators $A_{S}(X) \times$ $A_{T^{\prime}}(Y) \rightarrow A_{T \cap T^{\prime}}(Y)$, where $T^{\prime}$ is an arbitrary support in $Y$ and $S^{\prime}=f^{-1}\left(T^{\prime}\right)$;

3) invariance under a transversal change of the base: let $X, Y$, and $\tilde{Y}$ be smooth varieties, and let $f: X \rightarrow Y$ and $g: \tilde{Y} \rightarrow Y$ be transversal mappings (in this case $\tilde{X}=\tilde{Y} \times_{Y} X$ is a smooth variety, see Subsection 4.2.1); if $S \subset X$ and $T \subset Y$ are supports, $f(S) \subset T$, and $f$ is proper on $S$, then the following diagram is commutative:

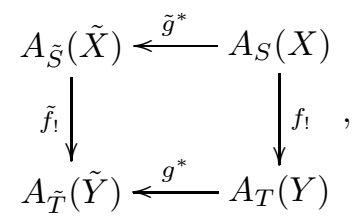

where $\tilde{f}=\tilde{Y} \times_{Y} f, \tilde{g}=g \times_{Y} X, \tilde{S}=(\tilde{g})^{-1} S$, and $\tilde{T}=(\tilde{f})^{-1} T$.

Sometimes, transfers are called "direct images" or "integrations". We note that transfers are defined for arrows that, in general, are not mappings of pairs.

3.1.2. Definition. Transfers are compatible with the orientation if $i_{\text {gys }}^{S, T}=i_{!}^{S, T}$ for every embedding $i: Y \rightarrow X$ of smooth varieties that is proper on an arbitrary support $S \subset Y$ and every support $T$ containing $S$.

Below (in Subsection 3.3.1), we prove that a theory can uniquely be equipped with transfers compatible with a given orientation, but first, we study the properties of transfers.

3.2. Properties of transfers. Here, we calculate transfers in several important cases. We calculate the transfer for the embedding of the scheme of zeros for a section of a vector bundle in terms of Chern classes, calculate the diagonal of a projective space, prove the Quillen formula [14 for the direct image under the structure projection of a projective bundle (therefore, the transfers are uniquely determined by a given orientation, since each mapping can be represented as a composition of an embedding and such a 
projection), and related formulas for the logarithm of the formal law and the invariant form. We also obtain a formula for the transfer under blowup of zeros of a section and compare the fundamental classes of the fibers in a family.

3.2.1. Theorem. Let $E$ be a vector bundle over a smooth variety $X, s$ a section of $E$, and $i: Y \subset X$ the embedding of the scheme of zeros of $s$. If $Y$ is a smooth subvariety of pure codimension $\mathrm{rk} E$, then $i_{\mathrm{gys}}(1)=e(E)$.

Proof. First, we prove the theorem for a line bundle $E$. Changing the bundle by a composition of affine bundles (see Subsection 4.4.4), we assume that $E=f^{*} \mathcal{O}_{\mathbf{P}^{n}}$ (1) and $f^{*} z=s$, where $f$ is a mapping $X \rightarrow \mathbf{P}^{n}$ and $z$ is a section of $\mathcal{O}_{\mathbf{P}^{n}}(1)$. Then $f$ is transversal to the divisor $H$ of zeros of $z$, and since the Gysin operator commutes with the change of the base (see Subsection 2.3.6), we obtain $i_{\text {gys }} \circ(f \mid Y)^{*}=f^{*} \circ \mathrm{in}_{\text {gys }}$, where in is the embedding $H \subset \mathbf{P}^{n}$. Since the orientation and the parameter determining the Chern classes (see Subsection 2.6.1) are compatible, we obtain $\operatorname{in}_{\text {gys }}(1)=e\left(\mathcal{O}_{\mathbf{P}^{n}}(1)\right)$, which proves the statement.

In the general case, we use the splitting principle and assume that $E=V+W$, where $V$ and $W$ are bundles of smaller ranks. Let $s=v+w$, and let $Z$ consist of all smooth points of codimension rk $V$ in the scheme of zeros of $v$. Then $Y \subset Z$, and, by induction, we have $\alpha_{\text {gys }}\left(1_{Y}\right)=e(W \mid Z)$, where $\alpha$ is the embedding $Y \subset Z$, and $\beta_{\text {gys }}\left(1_{Z}\right)=e(V)$, where $\beta$ is the embedding $Z \subset X$. Consequently, $i_{\text {gys }}(1)=\beta_{\text {gys }} \alpha_{\text {gys }}(1)=\beta_{\text {gys }}(e(W \mid Z))=$ $\beta_{\text {gys }}\left(e(W) 1_{Z}\right)=e(W) e(V)=e(E)$.

3.2.2. Corollary. We have $i_{\text {gys }}(1)=e\left(\mathcal{O}_{X}(Y)\right)$ for an embedding $i: Y \subset X$ of a smooth divisor.

Proof. Indeed, $\mathcal{O}_{X}(Y)$ is defined together with the section $\mathcal{O}_{X} \rightarrow \mathcal{O}_{X}(Y)$, the scheme of zeros of which coincides with $Y$.

3.2.3. Corollary. For a smooth embedding $i: Y \rightarrow X$ of smooth equidimensional varieties, we have $i^{*} \circ i_{\text {gys }}(1)=e\left(N_{Y} X\right)$.

Proof. Since $i_{\mathrm{gys}}=$ s.e. $\circ i_{\mathrm{gys}}^{Y}$ (see Subsection 2.3.1) and $i^{*} \circ$ s.e. $=i_{Y}^{*}$, where $i_{Y}$ is the embedding $(Y, \varnothing) \rightarrow(X, X-Y)$, it suffices to prove that $i_{Y}^{*} i_{\text {gys }}^{Y}(1)=e(N)$, where $N=N_{Y} X$. Since $i_{\text {gys }}^{Y}=$ nd $\circ \theta$ (see Subsection 2.3.1), it suffices to prove the statement for $X=N$, where $i=z$ is the zero section. For the bundle $E=p^{*} N$ on $N$, where $p: N \rightarrow X$ is a projection, we have the tautological section $s$, the zeros of which coincide with $Y$. By Theorem 3.2.1, we have $z_{\text {gys }}(1)=e(E)=p^{*} e(N)$, which completes the proof because $z^{*} p^{*}=$ id (see Subsection 1.3.5).

3.2.4. For $x=t \otimes 1, y=1 \otimes t \in A^{2,1}\left(\mathbf{P}^{\infty} \times \mathbf{P}^{\infty}\right)$, and the diagonal $\Delta: \mathbf{P}^{n} \rightarrow \mathbf{P}^{n} \times \mathbf{P}^{n}$, we have

$$
\Delta_{\text {gys }}\left(f \mid \mathbf{P}^{n}\right)=\frac{x^{n+1}-y^{n+1}}{x-y}\left|\mathbf{P}^{n} \times \mathbf{P}^{n}=\left(x^{n}+x^{n-1} y+\cdots+x y^{n-1}+y^{n}\right)\right| \mathbf{P}^{n} \times \mathbf{P}^{n} .
$$

To prove this, consider the section $s: p_{1}^{*} L \rightarrow p_{1}^{*} p_{2}^{*} V \rightarrow V / p_{2}^{*} L$ of the bundle $\mathcal{O}_{\mathbf{P} V}(1) \otimes Q$ on $\mathbf{P}^{n} \times \mathbf{P}^{n}$, where $Q=p^{*} V / L$ and $\mathbf{P}^{n}=\mathbf{P} V$. The scheme of zeros of $s$ coincides with the diagonal, whence $\Delta_{\text {gys }}(1)=e\left(\mathcal{O}_{\mathbf{P} V}(1) \otimes Q\right)$ (see Subsection 3.2.1). The exact sequence $0 \rightarrow \mathcal{O}_{\mathbf{P} \infty}(1) \otimes \mathcal{O}_{\mathbf{P} V}(-1) \rightarrow \mathcal{O}_{\mathbf{P} \infty}(1) \otimes V \rightarrow \mathcal{O}_{\mathbf{P} \infty}(1) \otimes Q \rightarrow 0$ on $\mathbf{P}^{\infty} \times \mathbf{P}^{n}$ implies (see Subsection 2.5.6) the relation $e\left(\mathcal{O}_{\mathbf{P} \infty}(1) \otimes Q\right) e\left(\mathcal{O}_{\mathbf{P} \infty}(1) \otimes \mathcal{O}_{\mathbf{P} V}(-1)\right)=e\left(\mathcal{O}_{\mathbf{P} \infty}(1) \otimes V\right)$. Since the multiplication on $e\left(\mathcal{O}_{\mathbf{P} \infty}(1) \otimes \mathcal{O}_{\mathbf{P}} \infty(-1)\right)$ is injective (see Subsection 2.5.12), we see that

$$
\Delta_{\text {gys }}(1)=\text { the restriction of } e\left(\mathcal{O}_{\mathbf{P}^{\infty}}(1) \otimes V\right) / e\left(\mathcal{O}_{\mathbf{P}^{\infty}}(1) \otimes \mathcal{O}_{\mathbf{P}} \infty(-1)\right) \text { to } \mathbf{P}^{n} \times \mathbf{P}^{n} .
$$


Recalling Subsection 2.7 .3 and using the relation $y^{n+1} \mid \mathbf{P}^{n}=0$, we obtain

$$
\Delta_{\text {gys }}(1)=\frac{x^{n+1}-y^{n+1}}{x-{ }_{\phi} y}\left|\mathbf{P}^{n} \times \mathbf{P}^{n}=\frac{x^{n+1}-y^{n+1}}{x-y} \frac{x-y}{x-{ }_{\phi} y}\right| \mathbf{P}^{n} \times \mathbf{P}^{n} .
$$

Identity (23) and the linearity of $\Delta_{\text {gys }}$ over $A\left(\mathbf{P}^{n} \times \mathbf{P}^{n}\right)$ (see Subsection 2.3.3) imply the relation

$$
\begin{aligned}
\Delta_{\text {gys }}\left(f \mid \mathbf{P}^{n}\right) & =\Delta_{\text {gys }} \Delta^{*}\left[\left(x-{ }_{\phi} y\right) /(x-y) \mid \mathbf{P}^{n} \times \mathbf{P}^{n}\right] \\
& =\Delta_{\text {gys }}(1)\left[\left(x-{ }_{\phi} y\right) /(x-y) \mid \mathbf{P}^{n} \times \mathbf{P}^{n}\right],
\end{aligned}
$$

which proves (24) if we take (25) into account.

3.2.5. The Quillen operator. Let $(X, U)$ be a smooth open pair, let $B=A\left(X \times \mathbf{P}^{\infty}\right)$, and let $M=A(X, U)$. Since the theory is orientable, the algebra $B$ is a one-dimensional formal $A(X)$-algebra (Subsection 4.5.1), so that we have the $A(X)$-algebra $\tilde{B}$ (Subsection 4.5.2) and the $\tilde{B}$-modules of differentials $\Omega_{A(X)} \tilde{B}$ and $\Omega_{M} \tilde{B}$ (Subsection 4.5.3). The latter are called the modules of meromorphic differentials and denoted by $\tilde{\Omega}_{X}$ and $\tilde{\Omega}_{X, U}$, respectively.

Let $E / X$ be a vector bundle of rank $n+1$, and $p: \mathbf{P} E \rightarrow X$ the projection. The composition

$$
B \stackrel{e\left(E \otimes \mathcal{O}_{\mathbf{P}} \infty(1)\right)^{-1} \omega_{\mathrm{inv}}}{\longrightarrow} \tilde{\Omega}_{X}[-2(n+1)](-n-1) \stackrel{\text { res }}{\longrightarrow} A(X)[-2 n](-n)
$$

(the residue res was defined in Subsection 4.5.4, and the Euler class is invertible in $\tilde{B}$ by Subsection 2.5.11) is zero on the image of $e\left(E \otimes \mathcal{O}_{\mathbf{P} \infty}(1)\right)$ (Subsection 4.5.6). Therefore (see Subsection 2.5.12), this composition induces the operator

$$
p_{\text {quil }}: A(\mathbf{P} E) \rightarrow A(X), \quad \operatorname{deg} p_{\text {quil }}=[2 n](n) .
$$

We also put $p_{\text {quil }}^{T}=M \otimes_{A(X)} p_{\text {quil }}$ (the identification in Subsection 2.4 .3 is taken into account), where $T=X-U$.

3.2.6. Theorem. Let a theory be oriented and equipped with transfers compatible with a given orientation (see Subsection 3.1.2). Then $\mathrm{pr}_{!}=\mathrm{pr}_{\text {quil }}$ for a bundle $E / X$ and the projection $\operatorname{pr}: \mathbf{P} E \rightarrow X$. In particular, for $\alpha \in A^{i, j}(\mathbf{P} E)$, the Quillen formula

$$
\operatorname{pr}_{!}(\alpha)=\operatorname{res} \frac{\tilde{\alpha} \omega_{\text {inv }}}{\left(t+{ }_{F} \lambda_{0}\right) \cdots\left(t+{ }_{F} \lambda_{n}\right)} \in A^{i-2 d, j-d}(X), \quad d=n-1, n=\operatorname{rk} E,
$$

is valid. Here $\tilde{\alpha}$ is an arbitrary element of $A\left(X \times \mathbf{P}^{\infty}\right)$, the restriction of which is $\alpha$, $E=\bigoplus L_{i}$, and $\lambda_{i}=c_{1}\left(L_{i}\right)$. More generally, $\mathrm{pr}_{!}^{S, T}=\operatorname{pr}_{\text {quil }}^{T}$, where $T$ is a support in $X$ and $S=\operatorname{pr}^{-1}(T)$.

Proof. For a trivial $E$, the statement reduces to the fact that $\operatorname{pr}_{!}(1)=a_{d}$, where $\omega_{\text {inv }}=$ $\left(1+a_{1} t+a_{2} t^{2}+\cdots\right) d t$ (see Subsection 2.7.4). By (24) and the fact that the orientation is compatible with the transfer, we obtain $\Delta_{!}(f \mid \mathbf{P} E)=\left(u^{d}+u^{d-1} v+\cdots+u v^{d-1}+v^{d}\right) \Delta_{!}(1)$, where $f d t=\omega_{\text {inv }}$, and $u$ and $v$ are the restrictions of $t \otimes 1$ and $1 \otimes t$, respectively, to $\mathbf{P} V \times_{X} \mathbf{P} V$. Applying the transfer $q$ ! to this equation, where $q: \mathbf{P} E \times \mathbf{P} E \rightarrow \mathbf{P} E$ is the projection to the second factor, and taking into account the fact that $q \Delta=\mathrm{id}$, we obtain $1+a_{1} t+\cdots+a_{d} t^{d}=q_{!}\left(u^{d}\right)+q_{!}\left(u^{d-1}\right) t+\cdots+q_{!}(u) t^{d-1}+q_{!}(1) t^{d}$. Since $\left\{t^{i}\right\}$ is a basis of $A(\mathbf{P} E)$ over $A(X)$, we obtain $q_{!}(1)=a_{d}$. Changing the base of $q$ with the help of a constant section $X \rightarrow \mathbf{P} E$, we complete the proof. In the general case, we may assume that $E$ is contained in a trivial bundle $V$ and that $V=E+F$, where $F$ is a sum of line bundles. Since we have proved the theorem for $V$, it suffices to verify the formula for $E$, assuming that it is valid for $E+L$ with a line bundle $L$. The projection $V \rightarrow L$ induces an operator $\mathcal{O}_{\mathbf{P} V}(-1) \rightarrow p^{*} L$, and thus, a section $s$ of the bundle $\mathcal{O}_{\mathbf{P} V}(1) \otimes p^{*} L$. Since the 
scheme of zeros of $s$ coincides with $\mathbf{P} E$, we see that $i_{!}=i_{\text {gys }}$ coincides with multiplication by $e\left(\mathcal{O}_{\mathbf{P} V}(1) \otimes p^{*} L\right)$ (see Subsection 3.2.1), where $i$ is the embedding $\mathbf{P} E \subset \mathbf{P} V$. By assumption, $q_{!}=q_{\text {quil }}$, where $q$ is the projection $\mathbf{P} V \rightarrow X$. Therefore,

$$
\operatorname{pr}_{!}=q_{!} i_{!}=\operatorname{res} \frac{e\left(\mathcal{O}_{\mathbf{P} V}(1) \otimes p^{*} L\right) \omega_{\mathrm{inv}}}{e\left(\mathcal{O}_{\mathbf{P}^{\infty}}(1) \otimes V\right)}=\operatorname{res} \frac{\omega_{\text {inv }}}{e\left(\mathcal{O}_{\mathbf{P}^{\infty}}(1) \otimes E\right)} .
$$

The last statement follows from the case where the support is $X$ and the fact that $p_{!}^{S, T}=p_{!} \otimes_{A(X)} A_{T}(X)$. This follows from Subsection 2.4.3 and linearity with respect to the base for $T^{\prime}=X$.

3.2.7. Corollary. Let $\left[\mathbf{P}^{n}\right]=p_{!}(1) \in A^{2 n, n}(\mathrm{pt})$, where $p$ is the projection $\mathbf{P}^{n} \rightarrow$ pt. Then

$$
\omega_{\mathrm{inv}}=\left(1+\left[\mathbf{P}^{1}\right] t+\left[\mathbf{P}^{2}\right] t^{2}+\cdots\right) d t .
$$

3.2.8. Corollary. For each homomorphism $R^{\bullet} \rightarrow K$, where $K$ is a $\mathbb{Q}$-algebra, and for the logarithm of the formal law $\phi \otimes_{R} \bullet K$, we have the Mishchenko formula

$$
\log _{\phi}(t)=t+\frac{\left[\mathbf{P}^{1}\right]}{2} t^{2}+\frac{\left[\mathbf{P}^{2}\right]}{3} t^{3}+\cdots
$$

3.2.9. Corollary. Let $X$ be a smooth variety, let $Y$ be a smooth subvariety of $X$, and let $\sigma: \hat{X} \rightarrow X$ be the blowup with center at $Y$. If $Y$ is the variety of zeros for a section $s$ of a vector bundle $E / X$ of rank $n=\operatorname{dim} X-\operatorname{dim} Y$, then

$$
\sigma_{!}(1)=\operatorname{res}\left[c_{n-1}(E)+c_{n-2}(E) u+\cdots+u^{n-1}\right] t^{-n} \omega_{\text {inv }}, \quad \text { where } u=-\iota(t) .
$$

Proof. There is an embedding $i: \hat{X} \rightarrow \mathbf{P} E$ identifying $\hat{X}$ with

$$
\left\{(x, l) \mid x \in X, l \text { is a line in } E_{x}, s(x) \in l\right\} .
$$

Thus, $\hat{X}$ is the variety of zeros of the section $s(x, l)=x \in E_{x} / l$ of the bundle $Q=$ $p^{*} E / \mathcal{O}(-1)$ on $\mathbf{P} E$, where $p$ is the projection $\mathbf{P} E \rightarrow X$ and $i_{!}(1)=e(Q)$ (see Subsection 3.2.1). Using the product formula of Subsection 2.5.4, we obtain $c_{n-1}(Q)=c_{n-1}(E)+$ $c_{n-2}(E) u+\cdots+u^{n-1}$, which proves the statement if we refer to the relation $\sigma_{!}(1)=p_{!} i_{!}(1)$ and the Quillen formula for $p_{!}$.

3.2.10. Theorem. Let $[Y] \in A^{-2 d,-d}(\mathrm{pt})$ be the transfer of $1 \in A^{0,0}(Y)$ under the projection $Y \rightarrow \mathrm{pt}$ of a smooth projective variety $Y$ of dimension d. Let $X$ be a smooth variety, and let $p: X \rightarrow \mathbf{P}^{1}$ be a flat projective morphism smooth over $0 \in \mathbf{P}^{1}$ and such that the fiber $p^{-1}(1)$ is a union of smooth divisors $D_{1}, \ldots, D_{n}$ with normal intersections. Then $[Y]=\sum a_{J}[D(J)]$, where $Y=p^{-1}(0), J$ runs over the subsets of $\{1, \ldots, n\}$, $D(J)=\bigcap D_{j}, j \in J$, and $a_{J}$ is the coefficient at the multipower $x^{J}$ in $x_{1}+_{\phi} \cdots+_{\phi} x_{n}$.

Proof. Since $i_{!}: A(\mathrm{pt}) \rightarrow A\left(\mathbf{P}^{1}\right)$ is injective, where $i: 0 \rightarrow \mathbf{P}^{1}$ is an embedding, it suffices to prove the relation $i_{!}[Y]=i_{!}\left(\sum a_{j}\left[D_{J}\right]\right)$. Since $i_{!}[Y]=p_{!} \tilde{i}_{!}\left(1_{X}\right)$, where $\tilde{i}$ is the embedding $Y \rightarrow X$, we obtain the above relation by applying $p_{\text {! }}$ to the equation $\tilde{i}_{!}(1)=c_{1}\left(\mathcal{O}_{X}(Y)\right)=\delta_{1}+_{\phi} \cdots+_{\phi} \delta_{n}$. Here, we use the relation $\delta^{J}=\tilde{i}(J)_{!}\left(1_{D(J)}\right)$, where $\delta_{j}=c_{1}\left(O_{X}\left(D_{j}\right)\right)$ and $\tilde{i}(J)$ is the embedding $D(J) \rightarrow X$.

3.3. Constructing transfers. The following theorem is one of the main results of the present paper.

3.3.1. Theorem. Every orientable theory can uniquely be equipped with transfers compatible with a given orientation (see Subsection 3.1.2). 
Proof. We assume that a mapping $f: X \rightarrow Y$ is proper on a support $S \subset X$ and that a support $T$ contains $f(S)$. Since $X$ is quasiprojective, we have an embedding in : $X \rightarrow \mathbf{P}^{m}$. The mapping $\alpha=f \times$ in : $X \rightarrow Y \times \mathbf{P}^{m}$ is proper on $S$ because the graph of in is closed. Thus, $f$ can be included into the commutative diagram $D$,

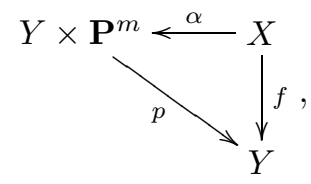

where all arrows are proper on the support (the support in $Y \times \mathbf{P}^{m}$ is $V=p^{-1} T \supset \alpha(S)$ ). Recalling Subsection 3.2.6, we obtain uniqueness.

To prove existence, we put $f_{!}^{S, T}(D)=p_{\text {quil }}^{T} \circ i_{\text {gys }}^{S, V}$ and verify that $f_{!}^{S, T}(D)$ does not depend on the choice of a decomposition. To this end, we include the diagrams $D_{m}$ and $D_{n}$ of type (26) constructed by the inclusions $X \rightarrow \mathbf{P}^{n}$ and $X \rightarrow \mathbf{P}^{m}$ into the commutative diagram

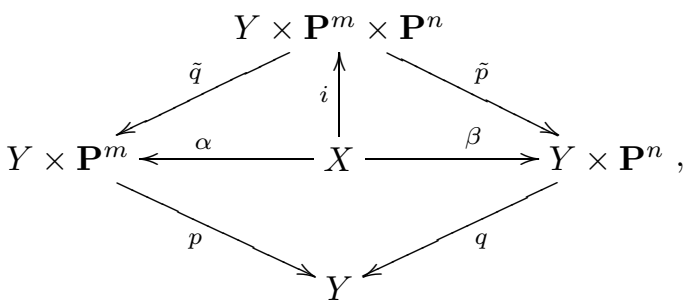

where $i=\alpha \times \beta, p, q, \tilde{p}$, and $\tilde{q}$ are projections, $V=p^{-1} T$, and $W=q^{-1} T$. The relation $f_{!}^{S, T}\left(D_{n}\right)=f_{!}^{S, T}\left(D_{m}\right)$ is a consequence of the formula

$$
q_{\text {quil }}^{T} \tilde{p}_{\text {quil }}^{W}=p_{\text {quil }}^{T} \tilde{q}_{\text {quil }}^{V}
$$

(if we take into account the isomorphism $A\left(\mathbf{P}^{n} \times *\right) \simeq A\left(\mathbf{P}^{n}\right) \otimes A(*)$, this can be deduced from the fact that the residue commutes with an operator acting on the coefficients, see Subsection 4.5.5) and the formula

$$
\alpha_{\text {gys }}^{S, V}=\tilde{q}_{\text {quil }}^{V}{ }_{\text {gys }}^{S, R} \quad \text { with } R=\tilde{q}^{-1} V .
$$

Relation (29) can be proved by using the diagram

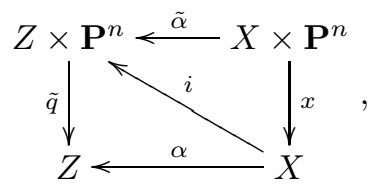

where $Z=Y \times \mathbf{P}^{m}$ and $x$ is a projection, or, more precisely, by using the relations

$$
\alpha_{\text {gys }}^{S, V}=\alpha_{\text {gys }}^{S, V} x_{\text {quil }}^{S} s_{\text {gys }}^{S, S \times \mathbf{P}^{n}}=\tilde{q}_{\text {quil }}^{V} \tilde{\alpha}_{\text {gys }}^{S \times \mathbf{P}^{n}, R} s_{\text {gys }}^{S, S \times \mathbf{P}^{n}}=\tilde{q}_{\text {quil }}^{V} i_{\text {gys }}^{S, R},
$$

where $s: X \rightarrow X \times \mathbf{P}^{n}$ is the section of $x$ constructed by the embedding $X \rightarrow \mathbf{P}^{n}$ used above in the construction of $\beta$ and $i$. The right identity in (30) follows from the functoriality of the Gysin operators (see Subsection 2.3.7); the middle identity follows from the fact that the Gysin operators are natural under a transversal change of the base $x: X \times \mathbf{P}^{n} \rightarrow X$ (Subsection 2.3.6) and the fact that the residue commutes with an operator acting on coefficients (Subsection 4.5.5). The left identity in (30) is a consequence of the relation

$$
x_{\text {quil }}^{S} s_{\text {gys }}^{S, S \times \mathbf{P}^{n}}=\mathrm{id} .
$$


To prove the latter, we represent $s$ by the change of the base of the universal section $\Delta$,

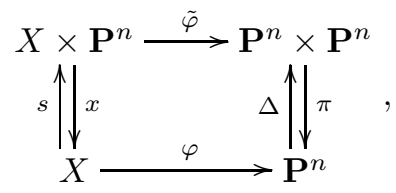

where $s=\mathrm{id} \times \varphi, \pi$ is the projection to the first coordinate, $\Delta$ is the diagonal, and $\tilde{\varphi}(P \times Q)=\varphi(P) \times Q$. Thus, since the Gysin operators are natural under a transversal change of the base $\varphi$ (Subsection 2.3.6), and the residue commutes with an operator acting on coefficients (Subsection 4.5.5), relation (31) reduces to the relation

$$
\pi_{\text {quil }}^{\bar{S}} \Delta_{\text {gys }}^{\bar{S}, \bar{S}} \times \mathbf{P}^{n}=\mathrm{id}
$$

where $\bar{S}$ is the closure of $\varphi(S)$ in $\mathbf{P}^{n}$. Since $\Delta_{\text {gys }}^{\bar{S}, \bar{S} \times \mathbf{P}^{n}}=\Delta_{\text {gys }}(1) \cdot \pi_{\bar{S}}^{*}$, where $\pi_{\bar{S}}^{*}$ is the operator $A_{\bar{S}}\left(\mathbf{P}^{n}\right) \rightarrow A_{\bar{S} \times \mathbf{P}^{n}}\left(\mathbf{P}^{n} \times \mathbf{P}^{n}\right)$ (this follows from the projection formula in Subsection 2.3.9 for $\Delta_{\text {gys }}$ and the fact that $\Delta^{*} \pi_{\bar{S}}^{*}=\mathrm{id}$ ) and $\pi_{\text {quil }}^{\bar{S}}=A_{\bar{S}}\left(\mathbf{P}^{n}\right) \otimes \pi_{\text {quil }}$ (see Subsection 4.5.5), we conclude that (32) reduces to the relation

$$
\pi_{\text {quil }} \Delta_{\text {gys }}(1)=1 .
$$

To prove (33), we use the relation $\Delta_{\text {gys }}(1)=e\left(Q \otimes L^{-1}\right)=c_{n}\left(Q \otimes L^{-1}\right)$ (see Subsection 3.2.1), where $Q=\tilde{V} / L, \mathbf{P}^{n}=\mathbf{P} V, L=\mathcal{O}_{\mathbf{P} V}(-1)$, and $\tilde{V}$ is the inverse image of $V$ under the projection $\mathbf{P}^{n} \rightarrow$ pt, because $Q \otimes L^{-1}$ has a section $s$ the zeros of which coincide with the diagonal $\left(s(x, y)\right.$ is induced by the operator $\left.L_{y} \subset V \rightarrow V / L_{x}\right)$. To calculate $c_{n}\left(Q \otimes L^{-1}\right)$, we represent $Q \otimes L^{-1}$ by the restriction to $\mathbf{P}^{n} \times \mathbf{P}^{n}$ of the bundle $Q \otimes L_{\infty}^{-1}$ on $\mathbf{P}^{n} \times \mathbf{P}^{\infty}$, where $L_{\infty}^{-1}=\mathcal{O}(-1)$. Moreover, $c_{n}\left(Q \otimes L_{\infty}^{-1}\right) c_{1}\left(L \otimes L_{\infty}^{-1}\right)=$ $c_{n+1}\left(V \otimes L_{\infty}^{-1}\right)$, and multiplication by $c_{1}\left(L \otimes L_{\infty}^{-1}\right)$ is an invertible operator $C \otimes_{B} \tilde{B} \rightarrow \tilde{B}$, where $B=A\left(\mathbf{P}^{n} \times \mathbf{P}^{\infty}\right)$ and $\tilde{B}$ is the localization defined in Subsection 3.2.5. The operator $C \otimes_{B} \tilde{B} \rightarrow \tilde{B}$ is invertible because $c_{1}\left(L \otimes L_{\infty}^{-1}\right)$ is an affine parameter by the criterion in Subsection 4.5.1 (we take Subsection 2.7.2 into account) and because $c_{1}(L)$ is nilpotent. Therefore, $\Delta_{\text {gys }}(1)$ is represented by the restriction of $c_{n}\left(Q \otimes L_{\infty}^{-1}\right)=$ $c_{n+1}\left(V \otimes L_{\infty}^{-1}\right) / c_{1}\left(L \otimes L_{\infty}^{-1}\right)=y^{n+1} /\left(y-{ }_{\phi} x\right)$ to $\mathbf{P}^{n} \times \mathbf{P}^{n}$, where $x=t \otimes 1$ and $y=1 \otimes t$ (see Subsection 2.7.2). Consequently,

$$
\pi_{\text {quil }} \Delta_{\text {gys }}(1)=\operatorname{res} \frac{\omega_{\text {inv }}}{y-{ }_{\phi} x}=\operatorname{res}_{u} \frac{\omega_{\text {inv }}}{u}=1,
$$

where $u=y-{ }_{\phi} x$; here the second equation is true because the residue is invariant (see Subsection 4.5.4), and the third equation follows from the fact that the $u$-expansion of $\omega_{\text {inv }}$ coincides with the $t$-expansion, because $\omega_{\text {inv }}$ is invariant and the $t$-expansion starts with 1. In other words, $\omega_{\text {inv }}=\left(1+a_{1} u+\cdots\right) d u=\left(1+a_{1} t+\cdots\right) d t$ (see Subsection 2.7.4).

Thus, we have proved relations (33), (32), and (31), and, with them, the fact that $f$ ! is well defined (does not depend on the choice of a diagram). The transfers constructed above are linear in base, invariant under a transversal change of the base (see Subsection 3.1.1), and compatible with a given orientation (see Subsection 3.1.2). Indeed, let $f$ : $X \rightarrow Y$ be an embedding, and let in $=j \circ f$ in (26), where $j$ is the embedding $Y \rightarrow \mathbf{P}^{m}$. Then $f_{\text {gys }}^{S, T}=f_{\text {gys }}^{S, T} s_{\text {gys }}^{T, \tilde{T}} p_{\text {quil }}^{T}=\alpha_{\text {gys }}^{S, \tilde{T}} p_{\text {quil }}^{T}=f_{!}^{S, T}$, where $s=\mathrm{id} \times j: Y \rightarrow Y \times \mathbf{P}^{m}$. Here, the first identity follows from (31), the second is a consequence of the functoriality of the Gysin operators, and the third is the definition of $f_{!}^{S, T}$. 
To complete the proof, it remains to verify that the composition of transfers coincides with the transfer of the composition $X \stackrel{f}{\rightarrow} Y \stackrel{g}{\rightarrow} Z$. We consider the embedding $i: X \rightarrow$ $\mathbf{P}^{n}$ and include it into the diagram

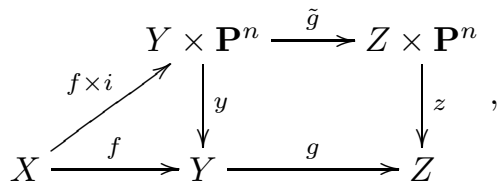

where $\tilde{g}=g \times \mathbf{P}^{n}$. Let $S, T$, and $R$ be supports in $X, Y$, and $Z$, let $f(S) \subset T$, and let $g(T) \subset R$. Using (35), the independence of transfers on the choice of a diagram, and the functoriality of the Gysin operators (see Subsection 2.3.7), we obtain $(g f) !$ $z_{\text {quil }}^{R} \tilde{g}_{\text {gys }}^{\tilde{R}, \tilde{T}}(f \times i)_{\text {gys }}^{S, \tilde{T}}$ and $f_{!}^{S, T}=y_{\text {quil }}^{T}(f \times i)_{\text {gys }}^{S, \tilde{T}}$, where $\tilde{R}=z^{-1} R$ and $\tilde{T}=y^{-1} T$. This reduces the above property of transfers to the relation

$$
z_{\text {quil }}^{R} \tilde{g}_{\text {gys }}^{\tilde{T}, \tilde{R}}=g_{!}^{T, R} y_{\text {quil }}^{T} \text {. }
$$

To prove (36), we consider an embedding $j: Y \rightarrow \mathbf{P}^{m}$ and the commutative diagram

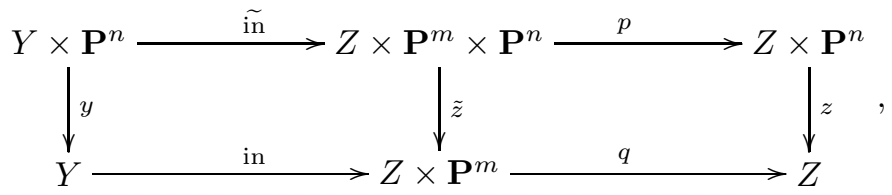

where in $=g \times j$, in $=$ in $\times \mathbf{P}^{n}$, and $\tilde{z}, p$, and $q$ are projections. From the relation $g_{!}^{T, R}=\operatorname{in}_{\text {gys }}^{T, V} q_{\text {quil }}^{R}$ for $V=q^{-1} R$ (transfers are independent of the choice of a diagram) it follows that the proof of (36) reduces to checking that the Quillen operators in the right square (see (28)) commute and that the Quillen operators commute with the Gysin operators in the left square, which follows from the fact that the residue commutes with an operator acting on coefficients; see Subsection 4.5.5.

We consider several examples.

3.3.2. $K$-theory (see Subsection 1.5.7). For a proper mapping of smooth varieties $f$ : $X \rightarrow Y$, we have a natural operator $f_{*}: K_{0}(X) \rightarrow K_{0}(Y),[E] \mapsto \sum(-1)^{p}\left[R^{p} f_{*} E\right]$, and therefore, the operator $f_{\beta}=\beta^{d} f_{*}: A^{0,0}(X) \rightarrow A^{0,0}(Y) \rightarrow A^{-2 d,-d}(Y)$, where $d=\operatorname{dim} Y-\operatorname{dim} X$. There is an orientation $\theta$ such that $f_{\beta}=f_{!}$(unique, because $t \mid \mathbf{P}^{n}=i_{\text {! }}(1)$ for the orienting parameter $t \in A^{2,1}\left(\mathbf{P}^{\infty}\right)$ and the linear embedding $i$ : $\left.\mathbf{P}^{n} \rightarrow \mathbf{P}^{\infty}\right)$. Since $\left[R^{0} i_{*}(1)\right]=[1]-[\mathcal{O}(-1)]$ and $i_{\beta}(1)=\beta^{-1}([1]-[\mathcal{O}(-1)])$, we see that the orienting parameter is defined by the relation $t=\beta^{-1} \lim \left([1]-\left[\mathcal{O}(-1) / \mathbf{P}^{n}\right]\right)$ (the system is compatible and the limit exists) and the orientation $\theta$ is determined by $t$ (for $\theta$ we have $f_{\beta}=f_{!}$). Moreover, $c_{1}(L)=\beta^{-1}\left([1]-\left[L^{-1}\right]\right.$ ) for a line bundle $L / X$, whence $c_{1}(L \otimes M)=c_{1}(L)+c_{1}(M)-\beta c_{1}(L) c_{1}(M)$, and the formal group law has the form $\phi(x, y)=x+y-\beta x y$, and $\omega_{\text {inv }}=(1-\beta t)^{-1} d t$.

3.3.3. Algebraic cobordism (see Subsection 2.2.10). Since $M U(\mathrm{pt})$ is the coefficient ring of the universal formal group law, we have a homomorphism $M U(\mathrm{pt}) \rightarrow \mathbf{M G L}^{\bullet}(\mathrm{pt})$. Since we know $M U(\mathrm{pt})$ and have transfers, we can prove that this map is injective. Thus, the coefficients $a_{i, j}$ of $\phi$ come from $M U(\mathrm{pt})$, and there are no new relations between them. 


\section{$\S 4$. Appendix}

In this section, we present some auxiliary material.

4.1. Categories with exact triangles. As a target category of a cohomology theory, we use an arbitrary additive category $\mathcal{A}$. The morphisms of $\mathcal{A}$ are called operators. We assume that $\mathcal{A}$ is equipped with a shift, i.e., with an invertible endofunctor $M \mapsto M[1]$ $(M[n]$ is the shift iterated $n$ times) and with a system of triangles, called exact (a triangle is a diagram $\Delta$ of the form $X \stackrel{u}{\rightarrow} Y \stackrel{v}{\rightarrow} Z \stackrel{w}{\rightarrow} X$ [1], where $v u=0, w v=0$, and $u[1] \circ w=0$, see [8]). We also assume that the given system of triangles is such that the triangles $X \stackrel{\text { id }}{\longrightarrow} X \rightarrow 0 \rightarrow X[1]$ are exact, a triangle isomorphic to an exact triangle is exact, a triangle is exact if and only if its shift $(\Delta[1]=(X[1] \stackrel{-u[1]}{\longrightarrow} Y[1] \stackrel{-v[1]}{\longrightarrow} Z[1] \stackrel{-w[1]}{\longrightarrow} X[2]))$ is exact, and the following conditions are satisfied:

1) the 5-lemma: a morphism of two triangles, i.e., a commutative diagram

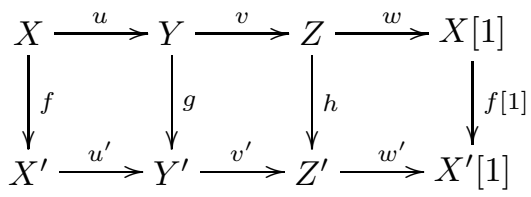

for which any two of the operators $f, g$, and $h$ are invertible is an isomorphism;

2) the sum of two triangles is exact if and only if each of the triangles is exact;

3) the cone of a morphism of two triangles is exact, where the cone of the morphism in (1) is the triangle

$$
Y+X^{\prime} \stackrel{\left(\begin{array}{cc}
-v & 0 \\
g & u^{\prime}
\end{array}\right)}{\longrightarrow} Z+Y^{\prime} \stackrel{\left(\begin{array}{cc}
-w & 0 \\
h & v^{\prime}
\end{array}\right)}{\longrightarrow} X[1]+Z^{\prime} \stackrel{\left(\begin{array}{cc}
-u[1] & 0 \\
f[1] & w^{\prime}
\end{array}\right)}{\longrightarrow} Y[1]+X^{\prime}[1]
$$

We note that, for a triangulated category with distinguished exact triangles, property $4.1,3$ ) is not always valid (the other properties were proved in $[8,1.1,1.2]$ ). However, this property is used only to prove that the triangle of a triple (see Subsection 1.3.3) and the triangle of Mayer-Vietoris (see Subsection 1.3.4) are exact. If, for a theory satisfying conditions 1)-3) in Subsection 1.2 .2 but taking values in a triangulated category, these triangles are exact, then the entire discussion after Subsection 1.3 .4 is applicable to this theory.

4.2. Deformation to the normal cone. This well-known construction [15] is very important for this paper, so we present it here in some detail. Since this construction is used only for smooth varieties, it is called deformation to the normal bundle or, simply, deformation to the normal.

4.2.1. Terminology. A closed embedding is a proper mapping $i: Y \rightarrow X$ of varieties inducing an isomorphism from $Y$ to its image $i(Y)$. In particular, if $Y$ is a smooth variety, then $i(Y)$ is a smooth subvariety of $X$.

For an embedding $Y \subset X$ of smooth varieties, the normal (normal bundle) is $N_{Y} X=$ $T_{Y} X / T Y$, where $T X$ and $T Y$ are the corresponding tangent bundles and $T_{Y} X$ is the restriction of $T X$ to $Y$.

Mappings $f: Y \rightarrow X$ and $g: Z \rightarrow X$ of smooth varieties are transversal if $d f+d g$ : $\tilde{g}^{*} T_{Y}+\tilde{f}^{*} T_{Z} \rightarrow p^{*} T_{X}$ is an epimorphism, where $\tilde{f}=f \otimes_{X} Z, \tilde{g}=Y \otimes_{X} g$, and $p=f \times_{X} g$. In this case, $P=Y \times_{X} Z$ is a smooth variety and $T_{P}=\operatorname{Ker}(d f+d g)$.

The normal is functorial. Indeed, if $f: \tilde{X} \rightarrow X$ is a mapping transversal to an embedding $Y \subset X$ of smooth varieties, then the differential $d f$ induces a mapping $N_{Y} f$ : $N_{f^{*} Y} \tilde{X} \rightarrow N_{Y} X$ of the normals and an isomorphism $N_{f^{*} Y} \tilde{X}=f^{*} N_{Y} X$. 
If $Z \subset Y$ and $i: Y \subset X$ are closed embeddings of smooth varieties, then the embedding $N_{Z} Y \rightarrow N_{Z} X$ of smooth varieties is defined as $N_{Z} i$.

4.2.2. Standard isomorphisms of normal bundles. For $Y$ embedded in $E$ by the zero section of the bundle, we have a natural isomorphism

$$
\nu: N_{Y} E \rightarrow E,
$$

because the derivation corresponding to a tangent vector gives a functional on $E^{*}$, i.e., a vector in $E$. The isomorphism $\nu$ identifying the normal to the zero section of the vector bundle with the vector bundle itself is used in the present paper without special notice.

For closed smooth embeddings $Z \subset Y \subset X$, we have the following natural isomorphisms of vector $Z$-bundles:

$$
N_{H} N_{Z} X \stackrel{\alpha}{\rightarrow} H \oplus F \stackrel{\beta}{\rightarrow} N_{Z} N_{Y} X
$$

where $H=N_{Z} Y, F=N_{Z} X / N_{Z} Y$, and the structure of a vector $Z$-bundle on $N_{H} N_{Z} X$ is not predetermined: $\alpha$ is constructed as an isomorphism of varieties and is used to introduce a vector structure on $N_{H} N_{Z} X$.

To describe $\alpha$ and $\beta$, we need the natural isomorphisms

$$
\left(N_{Y} X\right)\left|Z \simeq N_{Z} X / N_{Z} Y \simeq N_{Z} X / N_{Z} H \simeq\left(N_{H} N_{Z} X\right)\right| Z
$$

of vector $Z$-bundles, which allow us to identify $F$ with each of the above bundles if needed. The first identification in (40) is due to the fact that the natural projections occurring in the definition of the normal bundle, $\left(N_{Y} X\right) \mid Z \leftarrow T_{Z} X \rightarrow N_{Z} X / N_{Y} X$, have one and the same kernel $(T Y) \mid Z$. The second identification is implied by the fact that $N_{Z} Y$ coincides with $N_{Z} H$ in $N_{Z} X$. The third identification is similar to the first, with the replacement of $X$ by $N_{Z} X$ and $Y$ by $N_{Z} Y$.

Now, we describe the mappings in (39). The mapping $\beta$ is induced by the zero section $Y \subset N_{Y} X$ on $H$ and by the embedding $N_{Y} X \mid Z \subset N_{Y} X$ on $F=N_{Y} X \mid Z$ (see (40) above). The $H$-part of the mapping $\alpha: N_{H} N_{Z} X \rightarrow H \oplus F$ is the natural projection to $H$. The $F$-part of $\alpha$ is induced by the factorization $N_{Z} X \rightarrow N_{Z} X / H$.

The fact that all mappings mentioned above are invertible is checked locally.

4.2.3. The construction of a deformation to the normal. A deformation to the normal is constructed by a closed embedding $Y \subset X$ of smooth varieties and is used to compare the homotopy properties of the neighborhoods of $Y$ in $X$ and in the normal $N_{Y} X$. Since $N_{Y} X$ itself is not mapped to $X$, the best we can do here is to use an intermediate pair. Such a pair arises in the diagram

$$
\left(N_{Y} X, N_{Y} X-Y\right) \stackrel{s}{\rightarrow}\left(D_{Y} X, D_{Y} X-D_{Y} Y\right) \stackrel{g}{\leftarrow}(X, X-Y),
$$

where $s$ and $g$ are mappings of the special and typical fibers.

To describe (41), we consider the blowup $\sigma: \mathrm{Bl}_{Y \times 0}\left(X \times \mathbf{A}^{1}\right) \rightarrow X \times \mathbf{A}^{1}$ and put

$$
D_{Y} X=\mathrm{Bl}_{Y}\left(X \times \mathbf{A}^{1}\right)-\mathrm{Bl}_{Y}(X) \times 0 .
$$

The deformation to the normal $p: D_{Y} X \rightarrow \mathbf{A}^{1}$ is the composition of $\sigma$ and the projection $X \times \mathbf{A}^{1} \rightarrow \mathbf{A}^{1}$. Let $X_{1}=p^{-1}(1)$ and $X_{0}=p^{-1}(0)$. The fiber $X_{1}$ is said to be typical because the deformation of $p$ over $\mathbf{A}^{1}-0$ is trivial (trivialization is performed by $\sigma$ ).

The mapping $g$ of the typical fiber is the composition $X \rightarrow X \times 1 \stackrel{\sigma^{-1}}{\longrightarrow} X_{1} \subset D_{Y} X$. The mapping of the special fiber is the composition $s: N_{Y} X \subset N_{Y \times 0}\left(X \times \mathbf{A}^{1}\right) \rightarrow$ $\mathbf{P}\left(N_{Y \times 0}\left(X \times \mathbf{A}^{1}\right)\right)$, where $\mathbf{P}\left(N_{Y \times 0}\left(X \times \mathbf{A}^{1}\right)\right)$ is the exceptional divisor in $\mathrm{Bl}_{Y \times 0}\left(X \times \mathbf{A}^{1}\right)$. The image of $s$ lies in $D_{Y} X$ and identifies $N_{Y} X$ with $X_{0}$. 


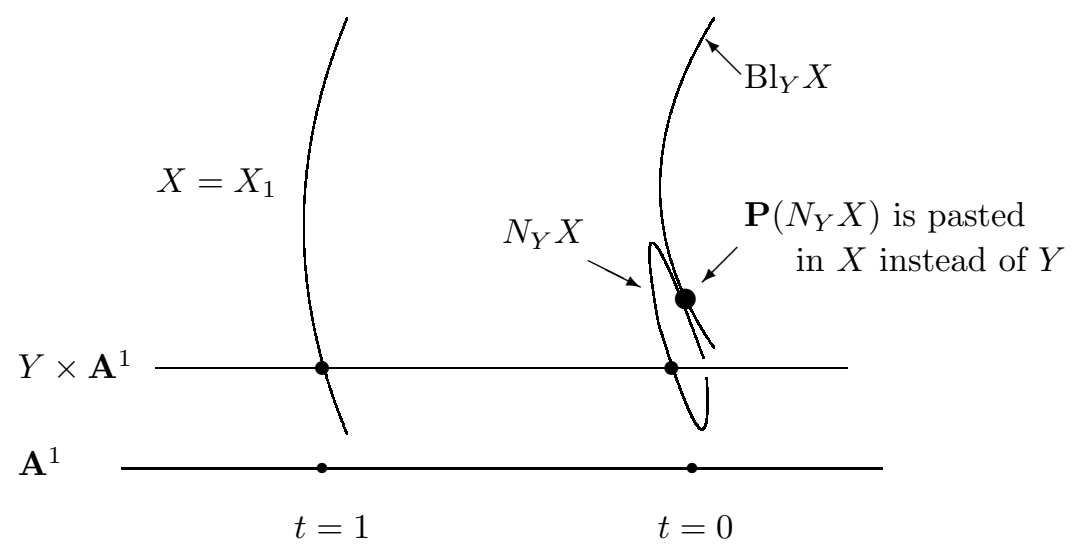

FiguRE 1.

The situation is explained below by depicting the projection $\mathrm{Bl}_{Y \times 0}\left(X \times \mathbf{A}^{1}\right) \stackrel{\sigma}{\rightarrow} X \times$ $\mathbf{A}^{1} \rightarrow \mathbf{A}^{1}$. Here, the exceptional divisor $\sigma^{-1}(Y \times 0)=\mathbf{P}\left(N_{Y \times \mathbf{A}^{1}}\left(X \times \mathbf{A}^{1}\right)\right)=\mathbf{P}\left(N_{Y} X+1\right)$ is $\mathbf{P}\left(N_{Y} X\right) \cup N_{Y} X$.

In Figure 1, $Y \times \mathbf{A}^{1}=D_{Y} Y$ (see Subsection 4.2.5) is embedded in $D_{Y} X$ by the functoriality of the $D$-construction (see Subsection 4.2.6).

Below, we present some properties of deformation to the normal.

4.2.4. The deformation $D_{Y} X \rightarrow \mathbf{A}^{1}$ is a smooth mapping. For obtaining this property, in particular, the subvariety $\mathrm{Bl}_{Y}(X) \times 0$ was cut out from the blowup (see (42)).

4.2.5. The deformation $D_{Y} Y$ is trivial, i.e., is isomorphic to $Y \times \mathbf{A}^{1}$. This follows from the fact that, for a smooth divisor $Y \times 0 \subset Y \times \mathbf{A}^{1}$, the blowup is trivial.

4.2.6. Deformation of a mapping. If $Y \subset X$ is a closed embedding of smooth varieties and the mapping $f: \tilde{X} \rightarrow X$ is transversal to this embedding, then there exists a mapping $D_{Y} f$ of deformations (over the base of deformations $\mathbf{A}^{1}$ ) such that the diagram

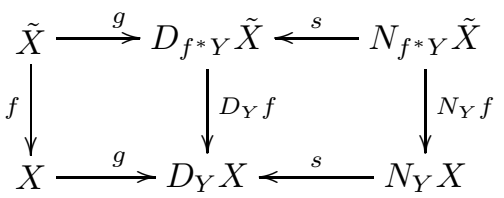

is commutative. This mapping is unique provided that it is defined as $f \times$ id over $\mathbf{A}^{1}-0$. This follows from the functorial property of blowups [15, B.6.9]. The mapping $D_{Y} f$ is called the deformation to the normal of the mapping $f$ and takes smooth mappings to smooth ones, proper mappings to proper ones, and embeddings to embeddings.

Moreover, both squares in (43) are fibered products. In other words, deformation to the normal is stable under a transversal change of the base (Subsection 4.2.1).

4.2.7. Deformation of a product. For closed embeddings $Y \subset X$ and $V \subset W$ of smooth varieties, there is an isomorphism $D_{Y \times V}(X \times W) \rightarrow D_{Y} X \times{ }_{\mathbf{A}^{1}} D_{V} W$ given by the mapping $D_{Y \times W} p \times D_{X \times V} q$, where $p$ and $q$ are the projections of $X \times W$ to its factors. 
4.2.8. Deformation of a vector bundle. Let $q: E \rightarrow Y$ be a vector bundle, and let $Z \subset Y$ be a closed embedding of smooth varieties. Then $D_{q^{*} Z} E$ has a natural structure of a vector bundle over $D_{Z} Y$ such that the structure mappings of the bundle $D_{q^{*} Z} E / D_{Z} Y$ (addition, multiplication by constants, ...) are the deformations to the normal (see Subsection 4.2.6) of the corresponding structure mappings of the bundle $E / Y$. The vector bundle $D_{q^{*} Z} E / D_{Z} Y$ is called the deformation to the normal of the bundle $E / Y$.

4.2.9. Trivialization of the normal to a deformation. For smooth closed embeddings $Z \subset Y \subset X$, there is a trivialization along $\mathbf{A}^{1}$,

(44) $N_{W} D_{Z} X \mid D_{Z} Z=q^{*}\left(N_{Y} X \mid Z\right)$, where $q$ is the projection $D_{Z} Z=Z \times \mathbf{A}^{1} \rightarrow Z$,

of the restriction of the vector bundle $N_{W} D_{Z} X$ to $D_{Z} Z=Z \times \mathbf{A}^{1}$.

Indeed, $N_{W} D_{Z} X=\sigma^{*}\left(N_{Y \times \mathbf{A}^{1}}\left(X \times \mathbf{A}^{1}\right)\right) \otimes \mathcal{O}\left(N_{Z} X\right)$ by [15, B.6.10]; here $\sigma: D_{Z} X \rightarrow$ $X \times \mathbf{A}^{1}$ is the blowup occurring in the definition of $D_{Z} X$. The first factor is constant along $\mathbf{A}^{1}$ by construction. We verify that the second factor is also constant. The line bundle $L=\sigma^{*} q^{*} \mathcal{O}_{\mathbf{A}^{1}}(0)$ is trivial (the coordinate $t$ on $\mathbf{A}^{1}$ gives a trivialization of $\mathcal{O}_{\mathbf{A}^{1}}(0) \subset$ $\left.\mathcal{O}\left(\mathbf{A}^{1}\right)\right)$. We consider the exceptional divisor $N_{Z} X$ on $D_{Z} X$ (the intersection of the exceptional divisor $\mathbf{P}\left(N_{Z} X+1\right) \subset \mathrm{Bl}_{Z \times 0}\left(X \times \mathbf{A}^{1}\right)$ and $\left.D_{Z} X\right)$. The restriction of this divisor to $D_{Z} Z$ coincides with $Z \times 0$, and therefore, the line bundle $\mathcal{O}\left(N_{Z} X\right) \mid D_{Z} Z$ is isomorphic to $L \mid D_{Z} Z$. Thus, this bundle is trivial and comes from $Z$.

Applying (44) to the embeddings $Z \subset Z \subset Y$, we obtain the isomorphism

$$
N_{D_{Z} Z} D_{Z} Y=q^{*} N_{Z} Y, \quad \text { where } q \text { is the projection } D_{Z} Z=Z \times \mathbf{A}^{1} \rightarrow Z,
$$

i.e., the normal is deformed trivially under deformation to the normal.

4.2.10. The deformation to the normal of the zero section of a vector bundle is trivial. Indeed, for a vector bundle $E / Y$, there is a natural isomorphism over $\mathbf{A}^{1}$ between the deformation diagram $E \stackrel{g}{\rightarrow} D_{Y} E \stackrel{s}{\longleftarrow} N_{Y} E$ and the diagram $E \stackrel{\text { id } \times 1}{\longrightarrow} E \times \mathbf{A}^{1} \stackrel{\text { id } \times 0}{\longleftarrow} E$ that is identical on the typical fiber and is inverse to the isomorphism $N_{Y} E \stackrel{\nu}{\rightarrow} E$ occurring in (38) on the special fiber. This isomorphism is a special case of the trivialization (44) for embeddings $Y \subset Y \subset E$ if we take the isomorphism $\nu$ into account.

4.2.11. Secondary deformation diagram. For closed embeddings $Z \subset Y \subset X$ of smooth varieties, we have the commutative diagram

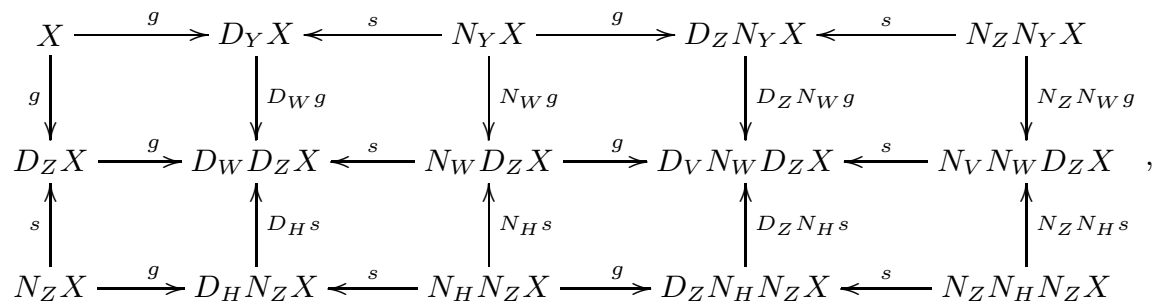

where $H=N_{Z} Y, W=D_{Z} Y, V=D_{Z} Z$, and the left column and rows consist of diagrams of deformations to the normal for the closed embeddings $N_{Z} Y \subset N_{Z} X, D_{Z} Y \subset$ $D_{Z} X, N_{Z} Y \subset N_{Z} X, Z \subset N_{Y} X$, and $D_{Z} Z \subset N_{W} D_{Z} X$ of smooth varieties. These embeddings are obtained by successive application of the constructions $N$ and $D$ (see Subsections 4.2.1 and 4.2.6) to the embeddings $Z \subset Y \subset X$. The vertical arrows are defined by successive application of the constructions $N$ and $D$ to the embeddings of the special and the typical fiber of the deformation $D_{Z} X$. Since diagram (43) is commutative, we see that diagram (46) is commutative. 
4.2.12. Trivializations in the secondary deformation diagram. The right column and the lower row of the right square in (46) are isomorphic to the rows and columns indicated in the diagram

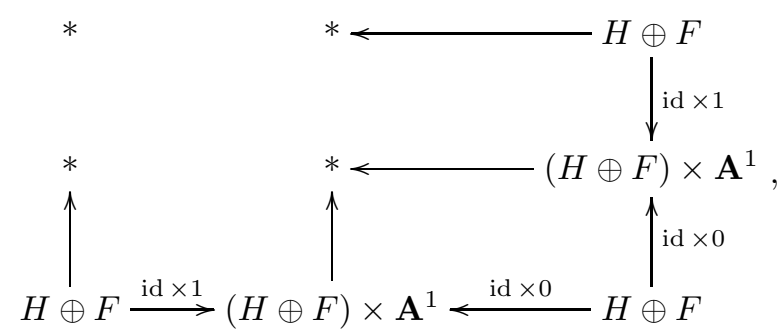

where $F=N_{Z} X / N_{Z} Y$. The identification $N_{H} N_{Z} X=H \oplus F$ coincides with the isomorphism of the secondary normal $\alpha$ in (39), and the identification $N_{Z} N_{Y} X=H \oplus F$ coincides with the isomorphism of the secondary normal $\beta$ in (39).

We describe the identifications but omit the proof (local calculations) that they are isomorphisms. A part of (46) "trivializes" in the direction of $N_{Z} Y$. More precisely, there is a natural embedding of the diagram

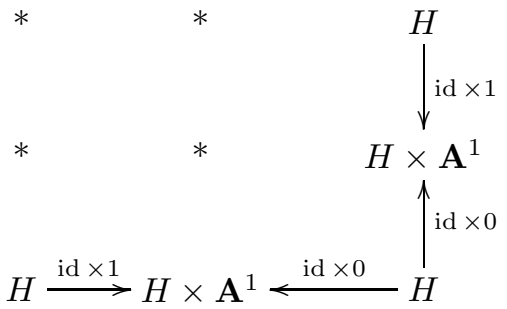

into the corresponding subdiagram of the right square of (46). Here, $H$ is included in $N_{H} D_{Z} X$, being the zero section of the $H$-bundle $N_{H} D_{Z} X$. Next, the application of $D_{Z}$ yields the embedding $H \times \mathbf{A}^{1} \subset D_{Z} N_{H} N_{Z} X$ (the deformation of the vector bundle $H / Z$ is trivial, see Subsection 4.2.101). Respectively, the special fiber of this embedding gives the embedding $H \subset N_{Z} N_{H} N_{Z} X$. The embedding $H \times \mathbf{A}^{1} \subset N_{V} N_{W} D_{Z} X$ is constructed as follows: $N_{V} N_{W} D_{Z} X \supset N_{V} N_{W} D_{Z} Y=N_{V} D_{Z} Y \supset N_{V} D_{Z} Z$, and this is $H \times \mathbf{A}^{1}$ by (45).

Moreover, the right square in (46) trivializes in the direction "orthogonal" to $N_{Y} Z$, i.e., the diagram

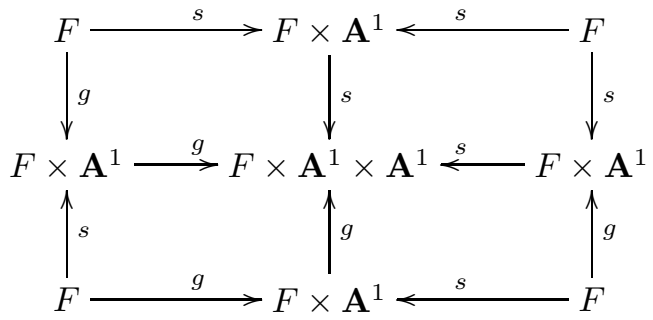

embeds into the right square of (46). Indeed, $N_{W} D_{Z} X$ contains $N_{W} D_{Z} X \mid D_{Z} Z$, which is identified with $F \times \mathbf{A}^{1}$ by Subsection 4.2 .9 (we recall that $F=N_{Z} Y \mid Z$, see Subsection 4.2.2). The other components of the right square are obtained from $N_{W} D_{Z} X$ by the constructions $N$ and $D$. Using the functorial property of these constructions, we obtain an embedding of (49) into the right square of (46).

At the positions where we have trivializations from (48) and (49) simultaneously, we obtain identification in (46), by using the structures of vector bundles over $Z$ or over 
$Z \times \mathbf{A}^{1}$. All these structures except the structure of a vector $Z$-bundle on $N_{H} N_{Z} X$ are natural. The latter was presented in Subsection 4.2.2

4.3. Infinite-dimensional constructions. Many results are stated naturally in terms of spaces such as $\mathbf{P}^{\infty}$, which, strictly speaking, are not varieties. To construct these spaces, the limits with values in the categories of ind- and pro-objects are used (see [16. 7.5] and [17, 8]).

4.3.1. Extension of a cohomology theory. We assume that a cohomology theory $A$ is extended to a functor Ind $\mathcal{S} \rightarrow \operatorname{Pro} \mathcal{A}$. Here, Ind $\mathcal{C}$ is the category of ind-objects of the category $\mathcal{C}$ (i.e., the inductive filtered systems $\left(X_{i}\right)$ ), and

$$
\operatorname{Hom}\left(\left(X_{i}\right),\left(Y_{j}\right)\right)=\underset{i}{\lim } \underset{j}{\lim } \operatorname{Hom}\left(X_{i}, Y_{j}\right) \text {. }
$$

A system $\left(X_{i}\right)$ as an object of $\operatorname{Ind} \mathcal{C}$ is denoted by $\lim X_{i}$. The category $\mathcal{C}$ is a full subcategory of Ind $\mathcal{C}$ consisting of systems indexed by a point.

Every functor $F: \mathcal{C} \rightarrow \mathcal{D}$ extends to a functor Ind $\mathcal{C} \rightarrow \operatorname{Ind} \mathcal{D},\left(X_{i}\right) \mapsto\left(F X_{i}\right)$, which is also denoted by $F$. The tensor and additive structures on $\mathcal{C}$ are naturally extended to Ind $\mathcal{C}$; for example, $\left(X_{i}\right) \otimes\left(Y_{j}\right)=\left(X_{i} \otimes Y_{j}\right)$. If $\mathcal{C}$ is an Abelian category, then so is Ind $\mathcal{C}$. The extensions of compatible tensor and additive structures are compatible.

Respectively, in $\operatorname{Pro} \mathcal{C}$, we have $\operatorname{Hom}\left(\left(X_{i}\right),\left(Y_{j}\right)\right)=\lim _{j} \lim _{i} \operatorname{Hom}\left(X_{i}, Y_{j}\right)$. A system $\left(X_{i}\right)$ as an object of Pro $\mathcal{C}$ is denoted by $\lim _{\longleftarrow} X_{i}$, and $\mathcal{C}$ is a full subcategory of Pro $\mathcal{C}$. Every cofunctor $F: \mathcal{C} \rightarrow \mathcal{D}$ extends to a cofunctor Ind $\mathcal{C} \rightarrow$ Pro $\mathcal{D}$ that has the tensor property and is additive. The morphisms of functors extend to morphisms of extensions.

4.3.2. Topology on $\operatorname{Hom}(X, Y)$. For $M=\lim M_{i}$ and $N=\lim _{\longleftarrow} N_{j}$ in ProC , the set $\operatorname{Hom}(M, N)=\lim _{j} \lim _{i} \operatorname{Hom}\left(M_{i}, N_{j}\right)$ is equipped with the limit topology, whereas $\operatorname{Hom}\left(M_{i}, N_{j}\right)$ has the discrete topology. The space $\operatorname{Hom}(M, N)$ is complete, and the topology is discrete for a finite projective system $N$ (for example, for $N \in \mathcal{C}$ ).

4.3.3. Infinite-dimensional Grassmannians. By definition, we have the equality $\mathbf{G r}_{r}=\underline{\lim } \mathbf{G r}_{r}(V)$, where the inductive system is formed by the inclusions of all finitedimensional subspaces $V$ of a countably-dimensional vector space (for limits, see Subsection 4.3.1); $\mathbf{P}^{\infty}=\mathbf{G r}_{1}$.

4.3.4. Vector bundles on Ind-varieties. A specialization of a vector bundle $E / X$ to a vector bundle $F / Y$ is a diagram

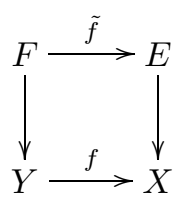

compatible with the vector structures. A vector bundle on an Ind-variety $\lim X_{i}$ is a projective system $\left(E_{i} / X_{i}\right)$ of specializations of vector bundles. It makes sense to speak of specializations of vector bundles on Ind-varieties.

4.4. Homotopy properties of vector bundles. In the theory of topological vector bundles, of significant importance is the splitting property of monomorphisms and epimorphisms and also the universal property of the tautological bundle on the Grassmannian. In algebraic geometry, none of the above makes sense (for example, the canonical embedding $\mathcal{O}(-1) \hookrightarrow \mathcal{O}^{2}$ on $\mathbf{P}^{1}$ does not split). Theorem 4.4 .1 below allows us to work with bundles in homotopic algebraic geometry as easily as in topology. We note that an affine bundle over a variety is a torsor of a vector bundle on this variety. By definition, 
the composition of affine bundles is a mapping of varieties that can be represented as a composition of structure projections of some affine bundles.

4.4.1. Theorem (see [18]). Let $E$ be a vector bundle over a variety $X$, and let $1^{n}$ be a trivial vector bundle of rank $n$ over $\tilde{X}$. Then:

1) the embeddings and epimorphisms of vector bundles are homotopically split: for each embedding $i: E \rightarrow F$ (epimorphism $p: F \rightarrow E$ ) of vector $X$-bundles, there exists an affine bundle $f: \tilde{X} \rightarrow X$ for which $f^{*} i$ (respectively, $f^{*} p$ ) is split;

2) for some compositions of affine bundles $f: \tilde{X} \rightarrow X$ and some $n$, there exists an epimorphism of bundles $1^{n} \rightarrow f^{*}(E)$; for some compositions of affine bundles $g: \tilde{X} \rightarrow X$ and some $m$, there exists an embedding of bundles $g^{*}(E) \subset 1^{m}$.

4.4.2. The universal bundle (see [18]). The tautological bundle $\tau$ on $\mathbf{G r}_{r}$ (see Subsection 4.3.3) formed by tautological bundles on finite Grassmannians is universal in the sense that (a) for a vector bundle $E / X$ of rank $r$, there exist a composition of affine bundles $p: \tilde{X} \rightarrow X$ and a mapping $f: \tilde{X} \rightarrow \mathbf{G r}_{r}(V)$ for some vector space $V$ such that $p^{*} E \simeq f^{*} \tau$; (b) if, for mappings $f_{0}: X \rightarrow \mathbf{G r}_{r}\left(V_{0}\right)$ and $f_{1}: X \rightarrow \mathbf{G r}_{r}\left(V_{1}\right)$, the bundles $f_{0}^{*}(\tau)$ and $f_{1}^{*}(\tau)$ are isomorphic, then there exists a mapping $h: X \times \mathbf{A}^{1} \rightarrow \mathbf{G r}_{r}(V)$ for some vector space $V$, and linear embeddings $j_{0}: \mathbf{G r}_{r}\left(V_{0}\right) \rightarrow \mathbf{G r}_{r}(V)$ and $j_{1}: \mathbf{G r}_{r}\left(V_{1}\right) \rightarrow$ $\mathbf{G r}_{r}(V)$ such that $h \times 0=j_{0} f_{0}$ and $h \times 1=j_{1} f_{1}$. In the same sense, the bundle $\tau^{\vee}$ is homotopically universal (apply the universality property of $\tau$ to $E^{\vee}$ ).

4.4.3. The characteristic operator. Though, in general, the bundle $E / X$ is not obtained from the tautological bundle by the mapping $j_{E}: X \rightarrow \mathbf{G r}_{r}, r=\mathrm{rk} E$, there is an operator (a ring operator for a ring theory)

$$
j_{E}^{*}: A\left(\mathbf{G r}_{r}\right) \rightarrow A(X)
$$

called characteristic and determined by the property that $j_{\tau}^{*}=$ id for the tautological bundle $\tau / \mathbf{G r}_{r}$ and $j_{F}^{*}=f^{*} j_{E}^{*}$ for each specialization $(f, \tilde{f}): E / X \rightarrow F / Y$. Recalling Subsection 1.3.5, we can construct $j_{E}^{*}$ by choosing (see Subsection 4.4.2) an affine bundle $p: \tilde{X} \rightarrow X$ and a mapping $u: \tilde{X} \rightarrow \mathbf{G r}_{r}(V)$ such that $p^{*} E \simeq u^{*} \tau$ and putting $j_{E}^{*}=p^{*}$. The required properties of the operators $j_{E}^{*}$ follow from Subsection 4.4 .2 .

4.4.4. The universal section. The section $\sigma$ of the bundle $\tau^{\vee} / \mathbf{G r}_{r}$ obtained by the composition $1 \subset 1^{\infty} \rightarrow \tau^{\vee}$ is homotopically universal in the sense that, for every section $s$ of a vector bundle $E / X$ of rank $r$, there is a composition of affine bundles $p: \tilde{X} \rightarrow X$ and a mapping $f: \tilde{X} \rightarrow \mathbf{G r}_{r}(V)$ for some vector space $V$ such that $p^{*} E \simeq f^{*} \tau^{\vee}$ and $p^{*} s=f^{*} \sigma$. It suffices to induce $f$ by the epimorphism $s \oplus \phi: 1+1^{n} \rightarrow E$, where $\phi$ is an arbitrary epimorphism $1^{n} \rightarrow E$, which exists by Subsection 4.4.1, under an appropriate change of the base.

4.4.5. For embeddings $i_{1}: E \rightarrow V_{1}$ and $i_{2}: E \rightarrow V_{2}$ of vector bundles on $X$, there exists a vector bundle $W$ and embeddings of vector bundles $\mathrm{in}_{1}: V_{1} \rightarrow W, \mathrm{in}_{2}: V_{2} \rightarrow W$, and $i: E \rightarrow W$ such that both $\mathrm{in}_{1} \circ i_{1}$ and $\mathrm{in}_{2} \circ i_{2}$ are homotopic to $i$ in the class of embeddings. In other words, there exist embeddings of bundles $h_{j}: p^{*} E \rightarrow p^{*} W$ over $X \times \mathbf{A}^{1}$, where $p: X \times \mathbf{A}^{1} \rightarrow X$ is a projection, such that the restriction of $h_{j}$ to $X \times 0$ coincides with $\operatorname{in}_{j} \circ i_{j}$ and the restriction of $h_{j}$ to $X \times 1$ is $i$. Indeed, we have $W=V_{1} \oplus V_{2}$, $i=i_{1} \oplus i_{2}, h_{1}=i_{1} \oplus t i_{2}$, and $h_{2}=t i_{1} \oplus i_{2}$.

4.4.6. For vector bundles $E \subset V$ and $F=V / E$ on $X$, the projection $U=\mathbf{P} V-$ $\mathbf{P} E \rightarrow \mathbf{P} F$ is equipped with the structure of the affine space of the vector bundle $q^{*} E \otimes$ $\mathcal{O}_{\mathbf{P} F}(1) \rightarrow \mathbf{P} F$, where $q$ is the projection $\mathbf{P} F \rightarrow X$. The action on $U$ is given on the fiber over $(x, l) \in \mathbf{P} F$, where $x \in X$ and $l$ is a line in the fiber $F_{x}$. The fiber $U_{x, l}$ consists of 
two lines $m \subset V_{x}$ projected onto $l$. For such an $m$, let $s$ be the inverse to the projection $l \rightarrow m \subset V_{x}$. The fiber $q^{*} E \otimes \mathcal{O}_{\mathbf{P} F}(1)$ consists of the arrows $f: l \rightarrow E_{x} ; f$ takes $m$ to the image of $l$ under $f+s$.

4.4.7. For vector bundles $F \subset V$ on $X$, and projections pr: $\mathbf{P} V \rightarrow X$ and $q: \mathbf{P} F \rightarrow X$, the mapping $\operatorname{pr}^{*} V \otimes O_{\mathbf{P} V}(1) \rightarrow T_{\mathbf{P} V / X}$ (see [15, B.5.8]) induces an isomorphism

$$
q^{*}(V / F) \otimes \mathcal{O}_{\mathbf{P} F}(1) \rightarrow N_{\mathbf{P} F}(\mathbf{P} V) .
$$

In particular, we have $N_{\mathbf{P} F}(\mathbf{P} V) \simeq V / F$ for a trivial one-dimensional bundle $F$.

4.4.8. For vector bundles $E, F$, and $V=E+F$ on $X$, there is an isomorphism

$$
\nu: N \rightarrow \mathbf{P} V-\mathbf{P} E, \quad N=N_{\mathbf{P} F} \mathbf{P} V
$$

given by the identification $N \simeq q^{*} E \otimes \mathcal{O}_{\mathbf{P} F}$ (1) (see Subsection 4.4.7), where $q$ is the projection $\mathbf{P} F \rightarrow X$, and by the identification of the affine bundle $\mathbf{P} V-\mathbf{P} E$ (see Subsection 4.4.6) with the vector bundle $q^{*} E \otimes \mathcal{O}_{\mathbf{P} F}(1)$ given by the section $\mathbf{P} F \subset \mathbf{P} V$ of the projection $\mathbf{P} V-\mathbf{P} E \rightarrow \mathbf{P} F$.

4.4.9. Decomposition of Pro $V$. For vector bundles $F \subset V$ on $X$ and the projection $V \rightarrow E=V / F$, we put

$$
P=\{(l, m) \in \mathbf{P} E \times \mathbf{P} V \mid \text { the projection of } m \text { to } E \text { lies in } l\} .
$$

The projections $p:(l, m) \mapsto l$ and $\sigma:(l, m) \mapsto m$ form the diagram $\mathbf{P} E \stackrel{\pi}{\leftarrow} P \stackrel{\sigma}{\rightarrow} \mathbf{P} V$, where $\sigma$ is the blowup of $\mathbf{P} V$ with center $\mathbf{P} F$ and $\pi$ is the projection $\mathbf{P} \tilde{F} \rightarrow \mathbf{P} E$, where the bundle $\tilde{F} \subset \operatorname{pr}^{*} V$ on $\mathbf{P} E$ is defined as the preimage of $\left.O_{\mathbf{P} E}(-1)\right) \subset p^{*} E$ under the projection $p^{*} V \rightarrow p^{*} E$ and $p$ is the projection $\mathbf{P} E \rightarrow X$. We have

$$
\mathcal{O}_{\mathbf{P} \tilde{F}}(-1)=\sigma^{*} \mathcal{O}_{\mathbf{P} V}(-1),
$$

because both bundles lie in $p^{*} V$ and their fibers at $(l, m)$ coincide with $m$.

In the case where $V=E+F$, the embedding $E \rightarrow V$ splits the projection $\tilde{F} \rightarrow$ $O_{\mathbf{P} E}(-1)$, so that $\tilde{F}=p^{*} F+O_{\mathbf{P} E}(-1)$. By Subsection 4.4.7, we have $N=p^{*} F \otimes O_{\mathbf{P} E}(1)$ for $N=N_{\mathbf{P} E} \mathbf{P} V$, whence $\tilde{N}=\tilde{F} \otimes O_{\mathbf{P} E}(1)$ for $\tilde{N}=N+\mathcal{O}_{\mathbf{P} E}$. Since $\mathcal{O}_{\mathbf{P} \tilde{F}}(-1) \otimes$ $O_{\mathbf{P} E}(1)=\mathcal{O}_{\mathbf{P} \tilde{N}}(-1)$ on $P=\mathbf{P} \tilde{F}=\mathbf{P} \tilde{N}$, formula (52) yields the following isomorphism:

$$
\mathcal{O}_{\mathbf{P} \tilde{N}}(-1) \simeq O_{\mathbf{P} E}(1) \otimes \mathcal{O}_{\mathbf{P} V}(-1) .
$$

4.5. Differentials and residues. Here, we present some facts about residues used in the main body of the present paper. Let $\mathcal{A}$ be a tensor additive category, and let $C$ be an invertible central (see Subsection 2.2.4) object (the assumption that $C$ is central allows us not to take care of permutations). We assume that $S \in \mathcal{A}$ is a commutative monoid, $B \in \operatorname{Pro} \mathcal{A}$ is a commutative $S$-algebra, $M$ is an $S$-module, $V$ is a $B$-module, $M[[C]]=M \otimes \lim _{\longleftarrow}\left[C^{0}+\cdots+C^{n}\right]$, and $M((C))=M \otimes \lim \lim _{\longleftarrow}\left[C^{-m}+\cdots+C^{n}\right]$ as $m, n \rightarrow \infty$, where the inductive system is given by the standard embeddings.

4.5.1. Affine parameters. An operator $u: C \rightarrow B$ is called an affine parameter for $B$ if the inductive system of operators $S \otimes\left[C^{0}+\cdots+C^{n}\right] \rightarrow B$ given by $u^{i}$ on $S \otimes C^{i}$ can be extended to an operator $\phi_{u}: S[[C]] \rightarrow B$ (this extension is unique) and $\phi_{u}$ is an isomorphism. An algebra $B$ is called a one-dimensional formal $S$-algebra if there exists an affine parameter for $B$.

For $B=S[[C]]$, there is a standard affine parameter $c$ given by the identity operator on $C$. We see that $u: C \rightarrow S[[C]]$ is an affine parameter if and only if the coefficient $s_{1}$ in the expansion $u=s_{1}+s_{0} c+s_{-1} c^{2}+\cdots$ is nilpotent and $s_{0}$ is invertible. 
4.5.2. Localization. By definition, a module $V$ localizes the affine parameters if, for every affine parameter $u$, the operator $C \otimes V \rightarrow V$ given by multiplication by $u$ is invertible. Let $\tilde{B}$ be a universal $B$-algebra belonging to Ind $\operatorname{Pro} \mathcal{A}$ (see Subsection 4.3.1) and localizing the affine parameters (if such an algebra exists). This means that, for every $V$ that localizes the parameters, there is a unique structure of a $\tilde{B}$-module on $V$ extending the structure of a $B$-module. A $\tilde{B}$-module $\tilde{V}$ is defined as $\tilde{\Lambda}=\Lambda \otimes_{B} \tilde{B}$ (if this product exists).

The algebra $\tilde{B}$ exists for a one-dimensional formal $S$-algebra $B$. Indeed, for $B=S[[C]]$ we take $\tilde{B}=S((C))$. By construction, $\tilde{B}$ localizes the affine parameter $s$, which implies the invertibility of all affine parameters because, in the expansion $u=s_{1}+s_{0} c+\cdots$, the coefficient $s_{1}$ is nilpotent and $s_{0}$ is invertible (see Subsection 4.5.1). In this case, the module $\tilde{V}$ is defined for $V=M[[C]]$ (we can take $\tilde{V}=M((C))$ ).

4.5.3. Differentials. Let $\Omega_{S} B$ be the module of differentials, i.e., the $B$-module of values of the universal $S$-linear derivation $d: B \rightarrow \Omega_{S} B$ (if it exists). For a module $M$, we have the constant $B$-module $\Omega_{M} B=M \otimes_{S} B$ and the integrable connection $d_{M}=M \otimes d: M \otimes_{S} B \rightarrow \Omega_{M} B$.

In particular, $\Omega_{S} B$ exists if $B$ is a one-dimensional formal $S$-algebra or its localization with respect to affine parameters. Indeed, for $B=S[[C]]$, we can put $\Omega_{S} S[[C]]=$ $S \otimes \lim _{\longleftarrow}\left[C+\cdots+C^{n}\right]$ and $d=n$ on the component $S \otimes C^{n}$. In this case, $\Omega_{M} B$ also exists since we can take $\Omega_{M} B=M \otimes \varliminf_{\lim }\left[C+\cdots+C^{n}\right]$. For $B=S((C))$, we put $\Omega_{S} S[[C]]=S((C))$ and $d=n$ on the component $S \otimes C^{n}$. In this case, $\Omega_{M} B$ also exists: we can put $\Omega_{M} B=M \otimes \lim \lim _{\longleftrightarrow}\left[C^{m}+\cdots+C^{n}\right]$.

4.5.4. Theorem (Invariance of the residue). For a one-dimensional formal $S$-algebra $B$ (see Subsection 4.5.1), there exists a unique operator res : $\Omega_{M} \tilde{B} \rightarrow M \otimes C^{-1}$ such that res $=\operatorname{res}_{u}$ for every affine parameter $u$, where $\operatorname{res}_{u}=\gamma_{-1} \circ \delta^{-1}$, the isomorphism $\delta: \Omega_{M} S((C)) \rightarrow \Omega_{M} \tilde{B}$ is induced by $\phi_{u}$ (see Subsection 4.5.1) in accordance with the universality property (see Subsection 4.5.3), and $\gamma_{i}$ is the $S$-linear projection $\Omega_{M} S((C)) \rightarrow M \otimes C^{i}$.

Proof. It suffices to prove that $\operatorname{res}_{u}=\operatorname{res}_{v}$ for affine parameters $u$ and $v$. Let $v=$ $\varepsilon+\beta u+\cdots$, where $\varepsilon$ is nilpotent and $\beta$ is invertible (see Subsection 4.5.1). This follows from $\operatorname{res}_{u}=\operatorname{res}_{w}=\operatorname{res}_{z}=\operatorname{res}_{v}$, where $w=z / \beta$ and $z=v-\varepsilon$. The relation $\operatorname{res}_{w}=\operatorname{res}_{u}$ is proved by the method indicated in [19, Remark at the end of II.11]. The relation $\operatorname{res}_{w}=\operatorname{res}_{z}$ is obvious. The operators $\operatorname{res}_{v}$ and $\operatorname{res}_{z}$ are trivial, and therefore, are equal on $\phi_{v} S[[C]]=\phi_{z} S[[C]]$. For negative exponents, the fact that $\operatorname{res}_{z}$ and res coincide follows from the explicit formula $z^{-n} d z=(v-\varepsilon)^{-n} d v=v^{-n}\left(1+\varepsilon v^{-1}+\varepsilon^{2} v^{-2}+\cdots\right)^{n} d v$ (the geometric sequence converges because $\varepsilon$ is nilpotent).

Geometrically, the residue of a form at a point is represented by an integral along a small contour around the point. Shifting the point by a quantity infinitesimally small in comparison with the formal parameter (nilpotent) does not take the point out of the small contour and so preserves the residue.

4.5.5. For an $S$-module $N$ and an $S$-linear operator $f: M \rightarrow N$, we have res $\circ \tilde{f}=$ $\Omega_{f} \circ$ res, where the operators $\tilde{f}: M((C)) \rightarrow N((C))$ and $\Omega_{f}: \Omega_{M} B \rightarrow \Omega_{N} B$ are induced by the action of $f$ on the coefficients. This follows directly from the definition of a residue.

4.5.6. The residue of an integer differential is zero, i.e., the composition $\Omega_{M} B \rightarrow \Omega_{M} \tilde{B} \stackrel{\text { res }}{\longrightarrow}$ $M \otimes C^{-1}$ (the first arrow is induced by the structure morphism $B \rightarrow \tilde{B}$ ) is trivial. This follows directly from the definition of a residue. 


\section{REFERENCES}

[1] F. Morel and V. Voevodsky, Homotopy category of schemes over a base, Preprint, 1997.

[2] V. Voevodsky, $\mathbf{A}^{1}$-homotopy theory, Proceedings of the International Congress of Mathematicians, Vol. 1 (Berlin, 1998), Doc. Math. 1998, Extra Vol. I, pp. 579-604. MR.1648048 (99j:14018)

[3] I. Panin and A. Smirnov, Push-forwards in oriented cohomology theories of algebraic varieties, $K$-Theory Preprint Archives, 459, 2000.

[4] J. S. Milne, Étale cohomology, Princeton Math. Ser., vol. 33, Princeton Univ. Press, Princeton, NJ, 1980. MR559531 (81j:14002)

[5] R. Thomason and T. Trobaugh, Higher algebraic K-theory of schemes and of derived categories, The Grothendieck Festschrift, Vol. 3, Progr. Math., vol. 88, Birkhäuser Boston, Boston, MA, 1990, pp. 247-435. MR.1106918 (92f:19001)

[6] R. Hartshorne, Algebraic geometry, Grad. Texts in Math., No. 52, Springer-Verlag, New YorkHeidelberg, 1977. MR0463157 (57:3116)

[7] A. Suslin and V. Voevodsky, Bloch-Kato conjecture and motivic cohomology with finite coefficients, The Arithmetic and Geometry of Algebraic Cycles, NATO Sci. Ser. C Math. Phys. Sci., vol. 548, Kluwer Acad. Publ., Dordrecht, 2000, pp. 117-189. MR1744945 (2001g:14031)

[8] A. Neeman, Triangulated categories, Ann. of Math. Stud., No. 148, Princeton Univ. Press, Princeton, NJ, 2001. MR1812507 (2001k:18010)

[9] M. Hovey, Model categories, Math. Surveys Monogr., vol. 63, Amer. Math. Soc., Providence, RI, 1999. MR1650134 (99h:55031)

[10] A. Smirnov, The Leibniz rule in algebraic K-theory, Zap. Nauchn. Sem. S.-Peterburg. Otdel. Mat. Inst. Steklov. (POMI) 319 (2004), 264-292; English transl., J. Math. Sci. (N. Y.) 134 (2006), no. 6, 2582-2597. MR2117861 (2005j:19001)

[11] D. Quillen, Higher algebraic K-theory. I, Algebraic K-Theory, I: Higher $K$-Theories (Proc. Conf. Seattle Res. Center, Battelle Memorial Inst., 1972), Lecture Notes in Math., vol. 341, Springer, Berlin, 1973, pp. 85-147. MR0338129 (49:2895)

[12] F. Morel, Basic properties of the stable homotopy category of smooth schemes, Preprint received approximately in March 2000.

[13] I. Panin, Push-forwards in oriented cohomology theories of algebraic varieties. II, K-Theory Preprint Archives, 619, 2003.

[14] D. Quillen, On the formal group laws of unoriented and complex cobordism theory, Bull. Amer. Math. Soc. 75 (1969), 1293-1298. MR0253350 (40:6565)

[15] W. Fulton, Intersection theory, Ergeb. Math. Grenzgeb. (3). Ser. Modern Surveys in Math., vol. 2, Springer-Verlag, Berlin, 1998. MR1644323 (99d:14003)

[16] P. Deligne, Catégories tannakiennes, The Grothendieck Festschrift. Vol. 2, Progr. Math., vol. 87, Birkhäuser Boston, Boston, MA, 1990, pp. 111-195. MR1106898(92d:14002)

[17] Théorie de topos et cohomologie étale des schémas. Tome 1. Théorie des topos, Séminaire de géométrie algébrique du Bois-Marie (1963-1964) (SGA4). Dirigé par M. Artin, A. Grothendieck et J. L. Verdier, Lecture Notes in Math., vol. 269, Springer-Verlag, Berlin-New York, 1972. MR 0354652(50:7130)

[18] A. Smirnov, Homotopy properties of algebraic vector bundles, Zap. Nauchn. Sem. S.-Peterburg. Otdel. Mat. Inst. Steklov. (POMI) 319 (2004), 261-263; English transl., J. Math. Sci. (N. Y.) 134 (2006), no. 6, 2580-2581. MR2117860 (2005i:14025)

[19] J.-P. Serre, Groupes algébriques et corps de classes, Publ. Inst. Math. Univ. Nancago, No. VII, Actualités Sci. Indust., No. 1264, Hermann, Paris, 1975. MR0466151 (57:6032)

St. Petersburg Branch, Steklov Mathematical Institute, Russian Academy of Sciences, Fontanka 27, St. Petersburg 191023, Russia

E-mail address: smirnov@pdmi.ras.ru

Received 10/JAN/2006

Translated by B. M. BEKKER 